

\section{(1) $2 \times$}

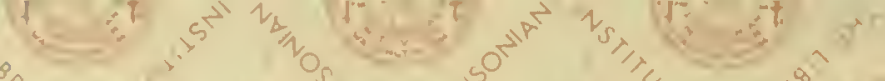

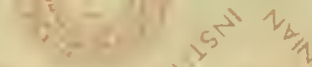

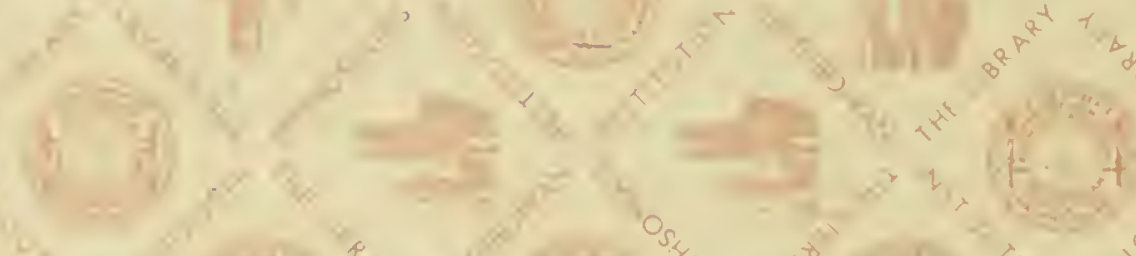

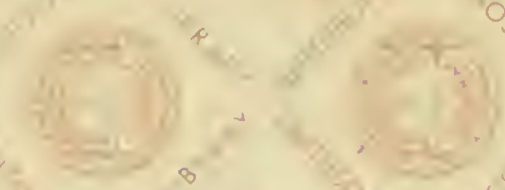

$\int_{0}^{2}+h^{2}+x^{2}=0=$

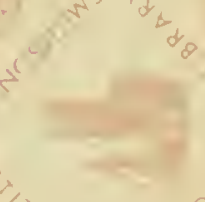

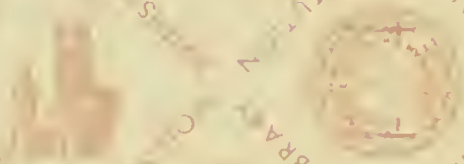

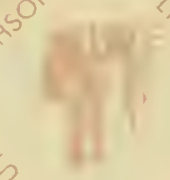

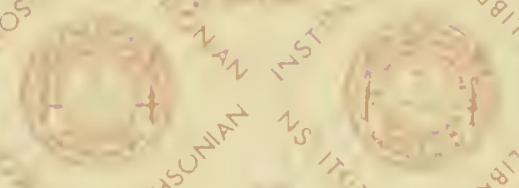

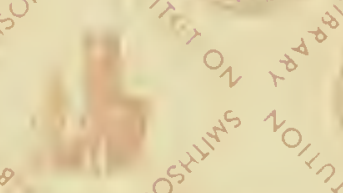

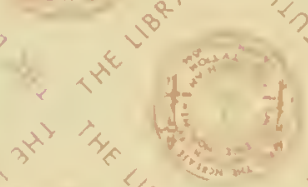

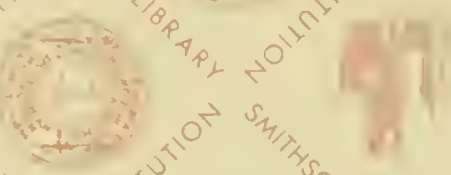

(3)

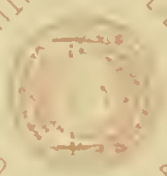

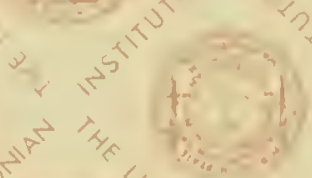

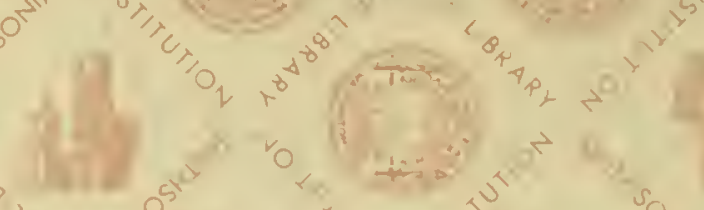

$4, y^{4}$
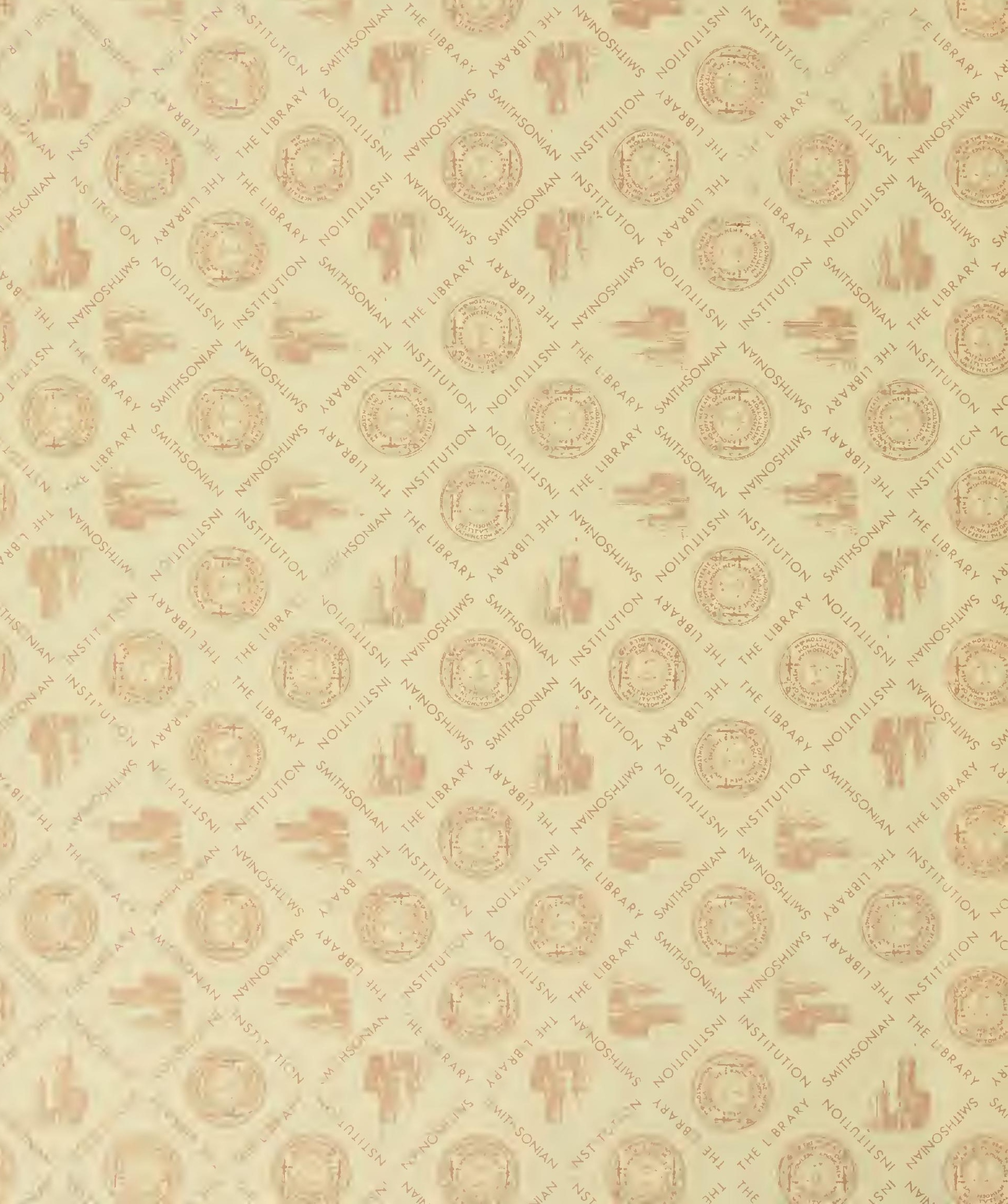

$\frac{1}{10}=$

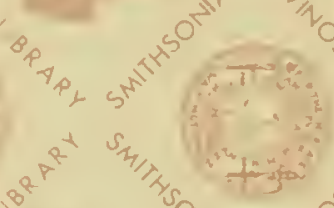

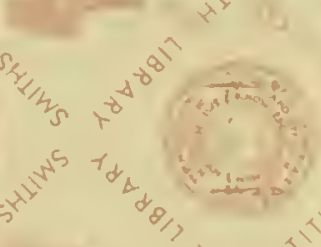

\&

(is)

Sall
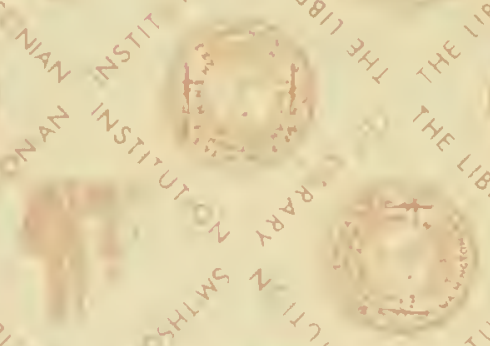

17.

(s)

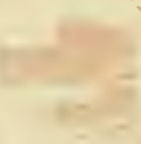

$1 \frac{1}{2}$

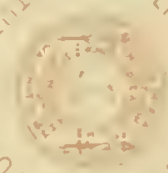

1. 9
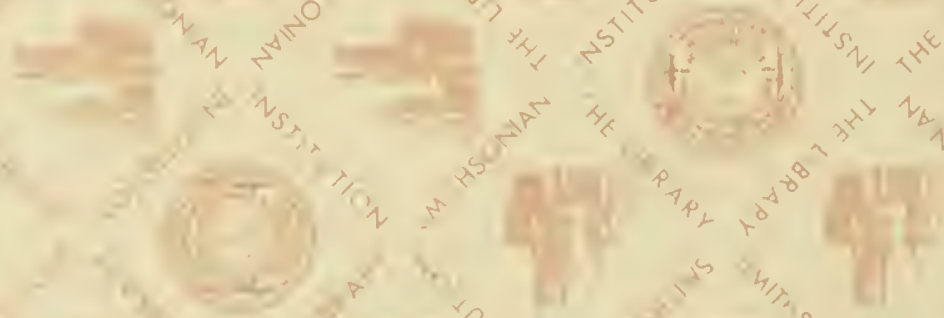

$\int^{6}$ itang

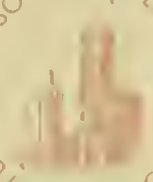




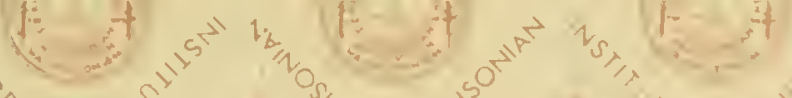

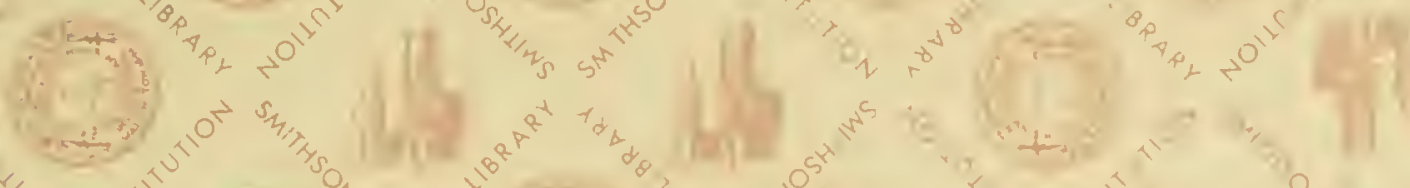

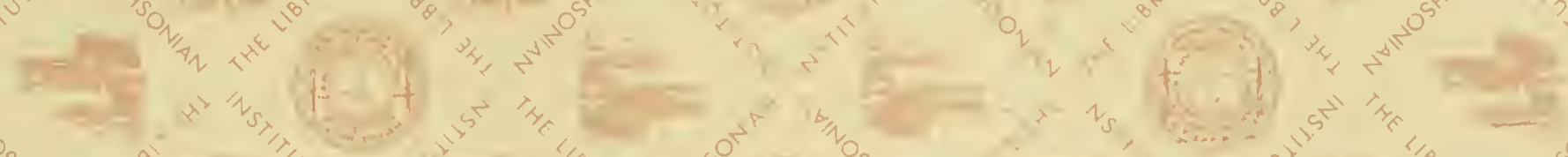

(1)

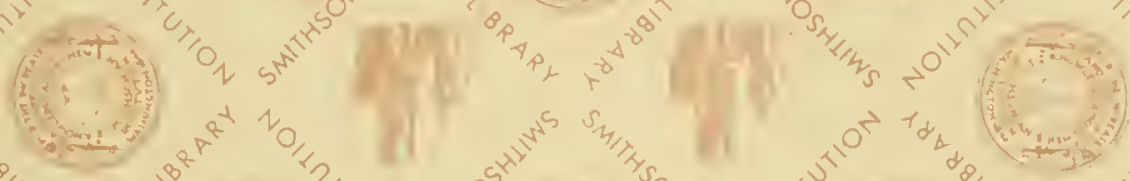

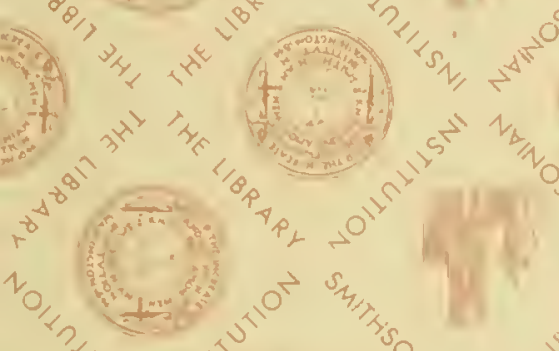

(t)

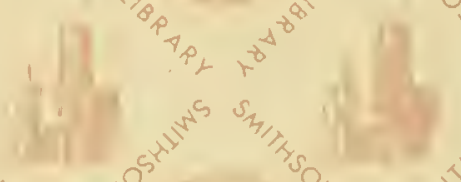

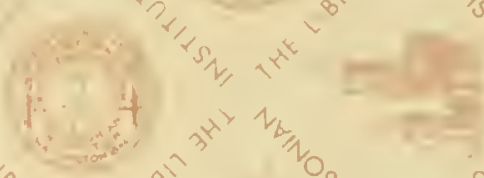

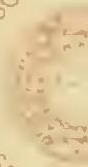

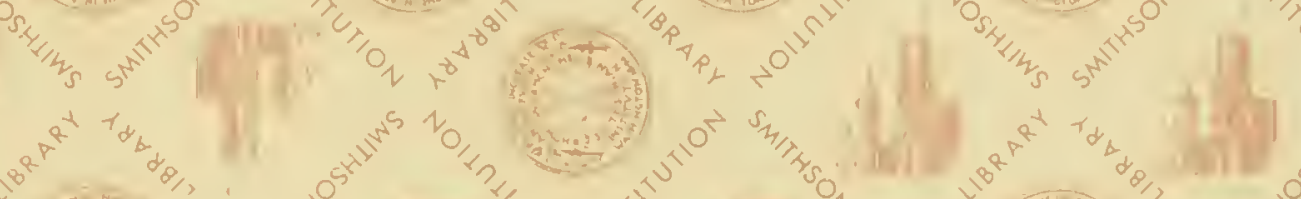

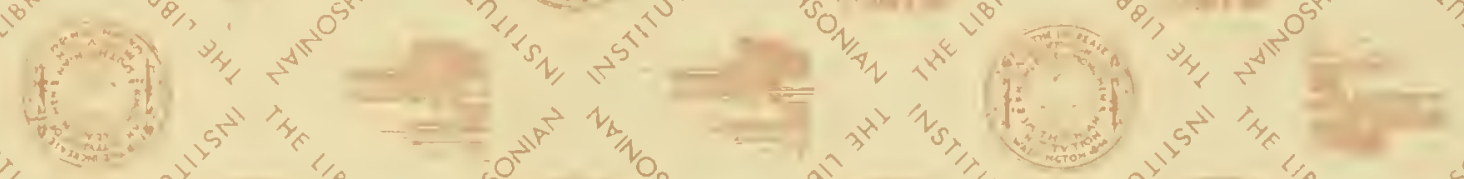

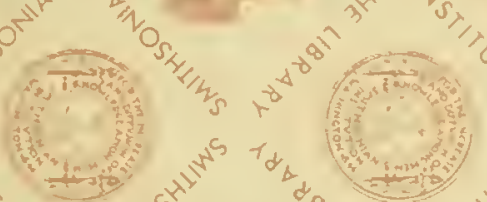

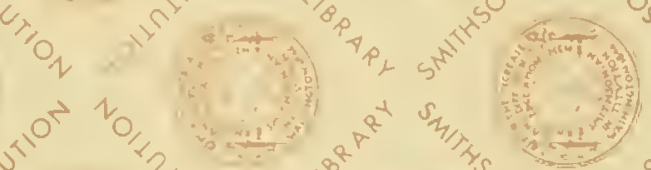

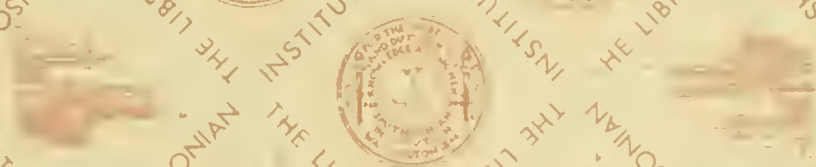

$=-\frac{1}{2}=$

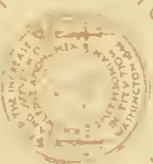

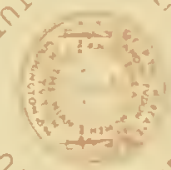

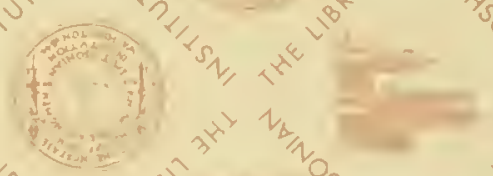

(1) $)^{2}$

1

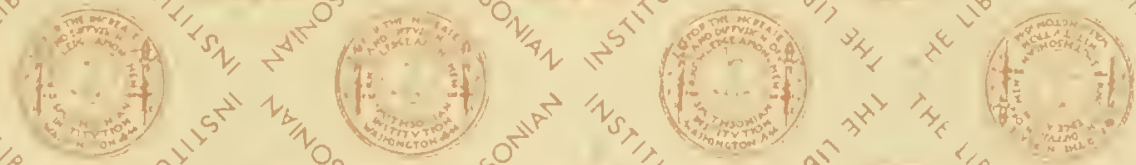

(5)

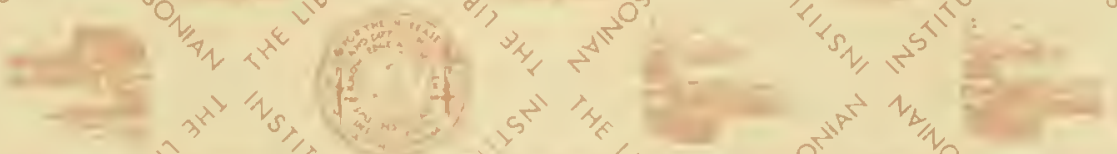

$x^{2}$

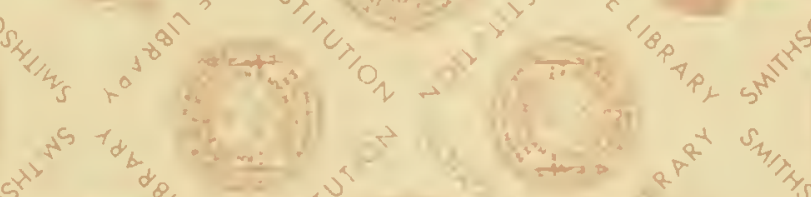

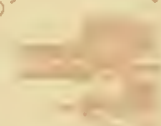
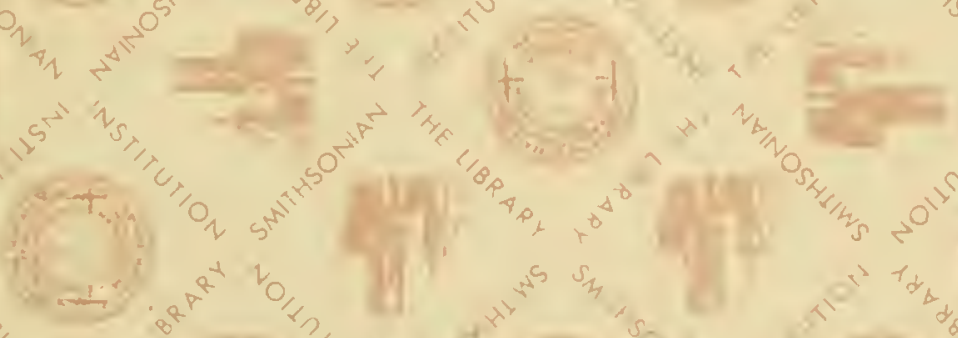

$1+\frac{1}{0}$

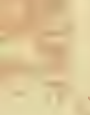

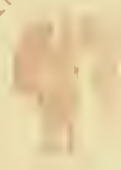

cititi

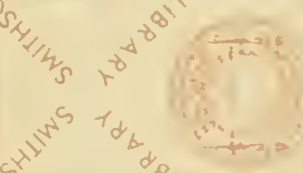

8
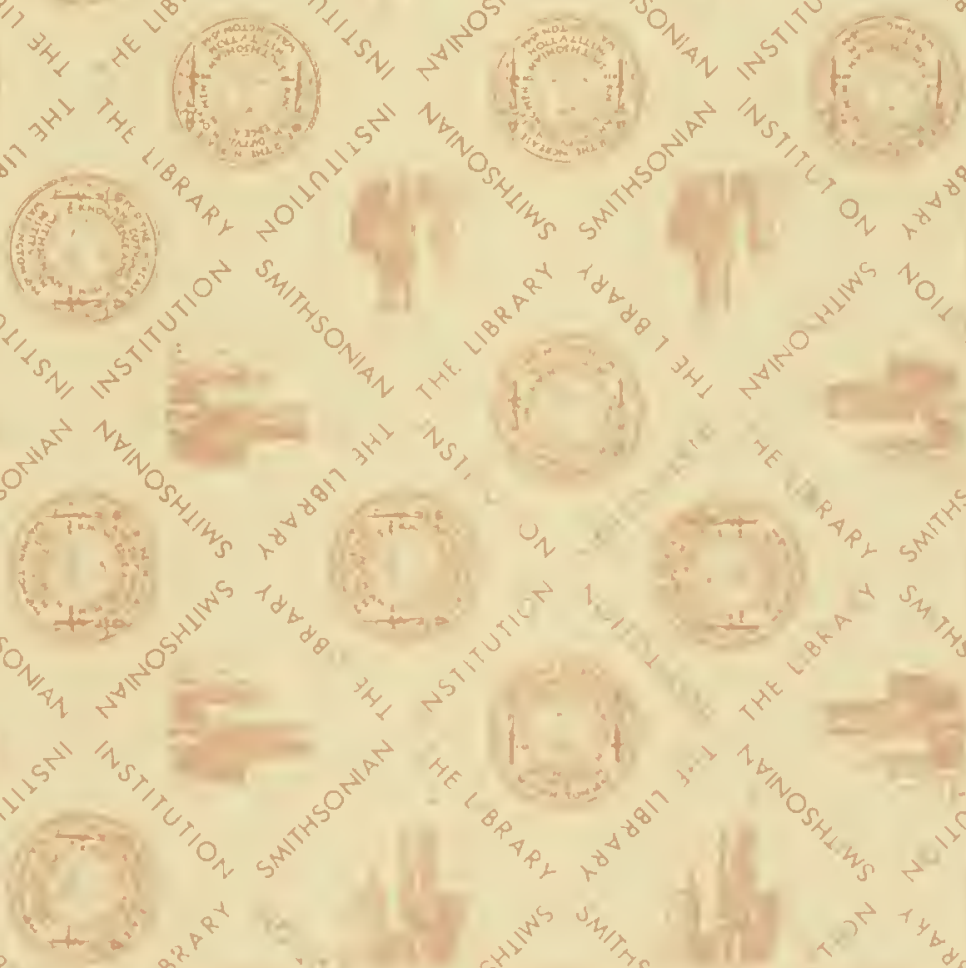



Fasc. III (Deuxième partie).

$\frac{\text { COPEOGNATHA }}{\text { Avec } 5 \text { Planches en couleurs }}$

\section{Collections Zoologiques}

DU BARON
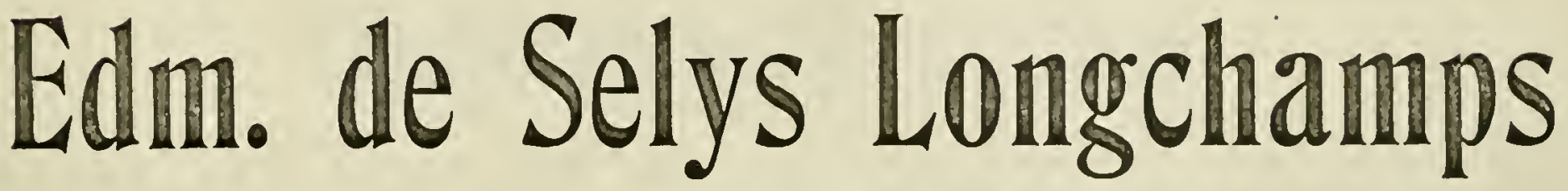

$\overline{704(2)}$

\section{CATALOGUE}

\section{SYSTEMATIQUE ET DESCRIPTIF}

\section{publie par les soins de ses liils,}

avec le concours de MM. M. Burr, J. Desneux, G. ENDERLEis,

F. Förster, J. Fraipont, K. Grünberg, F. Klapálek, R. Martin, F. Ris,

E. Rousseau, E. Petersen, Maurice de Selys LongChamps,

G. Severin, G. Ulmer et H. van der Weele.

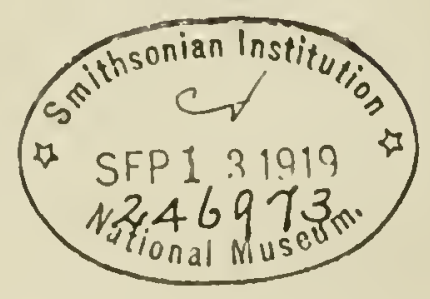

BRUXELLES 


\section{COPEOGNATHA}

VON

$D^{R}$ GÜNTHER ENDERLEIN, Stettin. 
Die Tafel und lextfiguren wurden von Dr G. ENDERLExix gezeichnet und von den Firmen IVERNER Und IVIXTER, Frankfurt a/m und Jea. MatVaUX of $C^{\circ}$. Brüssel, zum Druck hergestellt.

Die Namen der Spezies, welche sich aıcht in der Secrs'schen Sammlung befinden, sind zwischen Klammer [] gestellt. Ihre Beschreibung wurde in kleinen Characteren gedruckt. 


\section{COPEOGNATHA}

\section{EINLEITUNG}

Obgleich die Collection Selys eine Reihe für nomenklatorische Fragen wichtige Typen enthält, war es doch nicht angebracht, dieses Material als Basis für eine Monographie zu benutzen, da sonst ein zu grosses Missverhältnis der vorhandenen Arten zu den fehlenden auffallen würde. Zu einer Erleichterung der Uebersicht habe ich die curopiäischen von den aussercuropäischen Formen getrennt.

Für einige Arten habe ich zur sicheren Artfestlegung die weiblichen Copulationsorgane genau beschrieben und abgebildet. Es scheint, als ob bei den Copeognathen die weiblichen Genitalanhänge gute und sichere Artmerkmale abgeben; die männlichen Copulationsorgane sind meist schwieriger zu untersuchen und complicierter gebaut, aber sie sind ebenfalls für jede Species characteristisch gebaut. 



\title{
1. Die europäischen Copeognathen.
}

Die Collection Ser.ys ist besonders durch einige alte Typen von Latrenl.L, RAMbuR und Seurs wertroll und giebt riel wichtige Aufschlüsse über Synonymie. Zum Vergleich habe ich noch weitere Typen ron ReuTer und Nars ziehen kömen.

\author{
Subordo : ISOTECNOMERA.
}

Enderlein, Zool. Anz, 35. Bd., 1909, p. 172.

\section{FAM. PSOCIDAE.}

Genus PSOCUS LATr., i794.

Psocus Latreille, Bull. Soc. Philom., Vol. r, 1794 , p. 85.

Psocus gibbosus (Sulz. 1776).

Phryganea saltatrix Linné, Fama Sueciae. Ed. I., I 7 66, p. 226.

Phryganea gibbosa Sulzer, Abgek. Gesch. d. Insecten, 1776, p. 173, Taf. 24, Fig. I2 und $\mathrm{I}_{3}(\mathrm{q})$.

Hemerohius longicornis Fabricıus, Gen. Insect., 1777 (nicht r776), p. 245. - F. MÜLLEl, Prodr., I776, p. I $47, \mathrm{Nr} .1693$.

Psocus longicornis (F.) Latreille, Bull. Soc. Philom., I, r794, p. 85, Nr. 3. - (F). Fabricius, Ent. Syst., Suppl., I 798, p. 203.

Psocus lineatus Latreill.e, Coqueb. Ill. icon., I799, p. I 2, Taf. 2, Fig. 8 (sec. typ.!). - Latr.,

Stephens, Ill. Brit. Ent., i 836 , p. Ir9. - Burmeister, Handb., II, I839, p. 780 ,

$\mathrm{Nr}$. I8.

Psocus longicurnis (F.) Rambur, Neuropt., $18+2$, p. $320, \mathrm{Nr} .3$.

Psocus lineatus Latr., Brauer und Loew, Neuropteral anstriaca, I 857 , p. 36.

Psocus saltatrix (L.) Koube, Monogr. deutscher Psociden, I8So, p. 107.

Etc., etc.

Coll. Srises:

I O Paris (Coll. Latrenla:). I)ie Type von Psocus lineatus Latr.!; + ơ 9. Juli, 8 o (Coll. Ramusr); I q Juli; I q August 1861.

Belgien, Longchamps bei WVaremme, 25. Juni 3 ơ, 6 q; 25. Juli r O*, 3 q; Juli 5 ơ; August 1 \%; Halloy bei Ciney, 3 . September 1 .

Die Phrygrancu gibbosa Sulzer 1776 ist ganz sicher die Art, die bisher als Psocus longicarnis ( $\mathrm{F}$. ) geführt worden ist. Die Abbildung ist auf gar keme andere Art zu beziehen und ich setze daher für diese Species den Nanen Psocus gibbosus (Sulz.) ein. Die Grösse, Form, Färbung, sowie die langen Fühler der Sulzer'schen Abbildungen lassen dies berechtigt erscheinen. 


\section{Psocus nebulosus Steph., 1836.}

Psocus nebulosus Stephens, Illustr. Brit. Ent., 1836 , p. I99, Nr. 9 (q).

Psocus similis Stephexs, Illustr. Brit. Ent., i 836, p. I 20, Nr. Io (O').

Psocus ruriggatus Certis, Brit. Ent., i 836, p. 648 (nec Fabricius).

Psocus fuscipenuis 1) Alman, in Zerterstedt, Ins. Lappon., I 840 , p. 1053.

I'socus infuscatus Rambur, Neuropt., I8 42, p. $319, \mathrm{Nr} .1$ (q) (sec. typ.!).

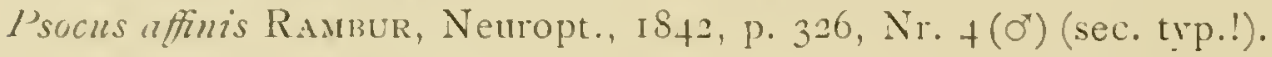

I'socus ncbuloso-similis Steph., Korlie, Mon. Deutsch. P'soc., is8o, p. 108 (ơo).

Etc., etc.

Coll. Selis: :

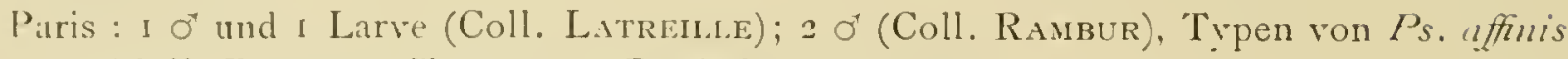
Ramis; 8 q (Coll. Ramuve, Typen von Ps. infuscutus Ramb.

Belgien : 2 q, Halloy bei Ciner, 25.7.1874.

Psocus bipunctatus (L., I 76 I).

Hemerabius bipunctutus Lixwé, Fama Suec., Ed. II, 176I, p. $3^{8} 4$.

I'sylla des pierres Geoffror; Hist. ins., I, I 762, p. $488, \mathrm{Nr} .7$.

Hemerobins bifunctatus L., Fabricies, Syst. Ent., I775, p. 310 , Nr. I I. -- L., Id., Spec. Ins., I 787 , I, p. 394, Nr. I3. - L., Schrank, Bayr. Reise, I786, p. 252. - L., Fabricius, Mantissa Ins., 1787, I, p. 247 , Nr. 16. - L., Id., Ent. Syst., II, 1793, p. 86, Nr. 23. L., Guei.in, Linvé, Syst. Nat., Ed. 1 3, 1793, p. 264 I, Nr. 27.

Psocus bipunctutus (L.), Latreil.te, Bull. Soc. Philom., I, I794, p. 85, Nr. 8. - (L.), Latreilie in Coguebert, Ill. Icon. Ins., Decas, I, I799, p. I 2, Tab. 2, Fig. 3. (l..), Walkinaer, Fann. Parisienne, II, i 802, p. 20, Nr. 3.

Hemerobius bipunctatus L., Schrank, Faun. Boica, T. 2, ISo4, p. I9I, Nr. I930.

Isocus bipunctatus (L.), Latreid.e, Hist. Nat., I, I 3, 1805, p. 73, Nr. 3.

Hemerobius hipunctutus L., TurTox, A general Syst. of Nat., I So6, Part. III, i 806, p. 403.

Psocus bipunctatus (L.), Panzer, Fann. Ins. Germ., Heft. 94, iso5, p. 2 I. - (L.), Samouelde, The Entomologists usefull compendium, I8I9, p. 85. - L., LEACH, Entomology, Brewster Edinb. Encyclop., I8 I5, 4, Vol. 9, Part. 1, p. I39, Nr. I. - (L.), Burmeister, Handb. Ent., II, I839, p. 779, Nr. I 4. - (L.), Curtis, Brit. Ent., I 823-I840, p. 648, Nr. I6. - (L..), Zetrerstedt, Ins. Lapp., I840, p. I053, Nota 5. - (L.), Rambur, Hist. nat. Ins. neuropt., I 842, p. 32 I, Nr.7. - (L.), Brauer nnd Loew, Neur, austr, 1857 , p. 33. - (L.), Mac Lachian, Ent. Mo. Mag., II, 1867, p. 243. - (L.), SpAingBerg, Psocina Snec. et Faun., I8 8 8, p. I 4 - - (L.), Korbe, Monogr. deutsch. Psociden, is8o, p. I09. - (L.), Kolre in Rostock, Neur. Germ., i 888, p. Iso. -- (L.), Tetens, Ent. Nachr., I7, I89I, p. 37 I and 375.

Etc., etc.

Coll. Seris :

Paris : 2 q (Coll. Latreil.te); i 5 q (Coll. Ramuur).

Belgien : 1 . 
Psocus quadrimaculatus L.ATR., I794.

(lafel I, Fig. 1-7.)

Psocus quadrimuculatus Latrente, Bull. Soc. Philom., I, I794, p. 85 (sec. typ.!).

I'socus quadrimaculatus Latr., Latreilie in Coquil., Ill. Icon., I799, p. iz, Taf. z, Fig. 6, 7 .

Psocus subuebulosus Stephens, I11. Brit. Ent., 1836, p. 12 I, Nr. It $\left(\mathrm{O}^{\top}\right)$.

I'socus maculipenuis Steprifes, I11. Brit. Ent., IS36, Vol. 6, p. I26 (q).

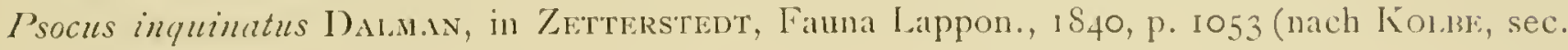
typ.).

Psocus conspurcatus Ramisur, Hist. Neuropt., 18+2, p. 323 (sec. typ.!).

Psocus quadrimaculatus Latr., Konle, Mon. Deutsch. I’sociden, isSo, p. I Io.

I'sucus hirticormis Reuter, Corrod. Fennica, IS93, pp. 1 1, 22, 4I, Fig. I (o'!) (sec. typ.!!).

I'socus subucbulosus Steph., Tetens, Ent. Nachr. (Karsci1), XVII, isq I, P. $375\left(O^{*}\right)$.

Psocus hilaris Navis, Revista Montsematina, Dec. I907, Fig. I ${ }^{\mathrm{n}-\mathfrak{c}}\left(\mathrm{O}^{\top}\right)$ (Spanien) (sec. typ!!).

Y'socus hilaris Nav., Navis, Neur. de Españay Portug., IgoS, p. $280\left(0^{*}\right)$.

Psocus quadrimaculatus Latr., Navis, Neur. de España y Portug., IgoS, p. aso (q).

Etc. etc.

Coll. Sei.ys :

Paris : 16 o (auf 7 Nadeln gespiesst) (Coll. Latremlif). 1)ie 'Typen von Latreille; I q (Coll. Rambur). Die Type von Psocus conspurcutus Rambur.

Die Typen von Reuter und Navás habe ich vergleichen können und danke auch an dieser Stelle diesen beiden Herren. Betreflend Psocus subuchulosus StepH. habe ich ein Stück zur Verfügung, das MaC LACHian mit den Typen von STEPHexs verglichen hat und es mit diesen identificierte.

Der ausserordentliche Dimorphismus und Dichromismus der beiden Geschlechter hat Stephens, Reuter und Navas immer wieder neu veranlasst, das ơ als besondere Species zu beschreiben. Die Zusanmengehörigkeit beider Geschlechter war bisher unbelannt.

In Juli Igoz fiel mir bei Berlin anf, dass ich trot der Häufigkeit der $q$ an einer Stelle keine $\sigma^{*}$ fand, die zu den $q$ passten. Einzelne ${ }^{*}$ darunter erschienen mir einer ganz anderen Art anzugehören. Durch eine Reihe weiterer Beobachtung konnte ich mich aber überzeugen, dass diese die $\sigma^{*} z u$ I's. quadrimaculutus LATr. sind, zumal dem o" sich ähnliche o durchaus nicht finden liessen.

o". Kopf mehr quer, hell bräunlich gelb. Clypeus ghatt, etwas rostbrännlich, ziemlich stirk gewölbt. Nitte des Scheitel melır rosthrann. Stemmaticum glänzend schwarz. Fühler etwa $t$ s so lang wie der Vorderllïgel oder noeh etwas mebr verlängert; blassbrännlich; Pubescenz der Geisselglieder sehr lang und senkrecht abstehend und ungefähr I 1/2 11 al so lang wie die 1)icke der Geissel. Iugen gross, schwarz, überhalblugelig abstehend. 
Thorax hell schmutziggelb; Mesonotum poliert glatt, bramschwar, braun oder wenigstens gebrännt (meist bei unausgefärbten Stüclien). Abdomen blass, die letzten Segmente stark chitinisicrt und dunkelbramn bis schwarzbram. Das 9. Stemit (Fig. $5 \mathrm{st}_{n}$ ) ist stark gewölbt und hinten mit kur\%er stmmpfer Gabel die eine kleine ovale Oeffmug unschliesst. Die Penishaken sind in Figur 5 ph sowje in Figur 6 abgebildet; dic Muskel sitzen an der Basis stark strahlenförmig und ferner bei $m$ an. Dorsale Telsonplatte (Fig. + lte) abgermndet dreieckig. Die Seitenklappen des Telson (lte) mit sehr grossem Sinnesfeld mucl imen mit kurzen zahnartigen Zäpfchen die nach oben gerichtet sind mnd am trockenen Tier mit starker Lupe auch erkamnt werden können (ebenso wie Form und Ausschnitt der Subgenitalplatte st ${ }_{9}$ ). Beine sehr blass, Schienen und Tarsen mit brämnlichem Antlug.

Flügel (Fig. I) hralin olne Zeichnma, selten mit Spuren der Flecken des weiblichen Vorderflïgels, die sich auch noch damn meist auf die Basalhälfte des Vorderflügels beschränken. Pterostigma und Hypostigmalsam blassbram bis hellbram, die Basalhälfte meist etwas heller. Scheitel des Pterostigma abgerundet. Radialramus und Nedia eine mehr oder weniger lange Strecke rerschmolzen, selten in einem P'unkte, noch seltener anch beide durch ()uerader verbunden. $\mathrm{r}_{++}$; fast doppelt so lang wic der Stiel. Scheitel der Areola postica breit. $\mathrm{cuz}_{2}$ lang und schrïg den Hinterrand treffend. Anali und Axillaris meist kur\% vor dem Nodulns vereinigt. Die Subcosta endet etwa in die Mitte von r, das Endstïck ist aber stark rerblasst.

Q. Körperfärbung wie beim ${ }^{*}$. Angen klein, schwarz, höchstens halbkugelig abstehend. Fühler liurz, höchstens ${ }^{\dagger} /$ der l'orderflïgeliänge; die letzten Glieder ungewöhnlich kurz; das 3. Glied lang; Pubescenz kurz, schräg abstehend.

Subgenitalplatte (Fig. + st $_{-+8}$ ) in der Mitte lamellenartig nach hinten verlängert, am Ende abgerundet. Gonopode des 8 . Segmentes (Fig. $+\mathrm{gp}_{8}$ ) stilettfömig; Mediangonopode des 9. Segmentes (Fig. 4 mg $\mathrm{p}_{9}$ ) lamellenartig verbreitert, Enddrittel stiletfömig innen mit längsreihe feiner kurzer basalwärts gerichteter Härchen. Lateralgonopode des 9. Segmentes $\left(\right.$ Fig. $+\lg \mathrm{p}_{g}$ ) abgerundet dreieckig. Die Telsonteile (Fig. + dte mnd lte) ähnlich wie beim ơ, die 1)orsalplatte grösser.

Flügel (Fig. 2) hyalin mit einigen brannen Fleckenzeichnungen, wie sie die Figur zeigt. Der Fleck in Zelle Cu und RR rerblasste häufig. Der Pterostigmafleck und der der Areola postica sind meist die kräftigsten. $\mathrm{r}_{++. ;}$ist öfter an der Basis stärker gebogen als beim $\sigma^{\top}$. $\mathrm{cu}_{2}$ ist meist noch wesentlich länger und anch noch mehr schräg gestellt und erzengt so eine ungewöhnliche Form der Areola postica, die für diese Spezies sehr characteristisch ist. Die Spitze und das Basaldrittel des l'terostigma ist meist weisslich, wie anch die üblichen Aderteile. Im Hinterflïgel ist die Spitze der Analzelle und die Mitte des Randsammes der Cubitalzelle schwach bis sehr schwach gebrämnt.

Körperlänge (in Alkohol) ơ 2,5-2, $8^{\mathrm{mm}} \cdot$ o $3-3,2^{\mathrm{mm}}$.

Vorderflïgellänge $\sigma^{7} z, 8-t^{\mathrm{mm}} \cdot q^{2} 2,7-3,8^{\mathrm{mm}}$ (die erstgenannten Grössen sind selten).

An dieser Stelle füge ich ein Stück ein, das ans der Collection Latrenl.te stammt und das nach der Fundnotiz von LATREILl,:s Hand aus Capland stammen soll; auf dem Originalzettel steht: (Cap de B. Esp.). Es hat die Form des ơ von Psocus quadrimaculatus Latr., aber die Flïgelfärbung (Fig. 3) ist die eines $q$, wenn auch etwas blasser. D)ie Füllerpubescenz ist etwas weniger steil. Ich vermute, dass es sich bei diesem Stïck mm eine Etikettenrerwechselung oder um eine falsch etikettiertes Stïck handelt, dass es also ein Pariser Stiick ist mit einer etwas abweichenden Färbung. Auf alle Fälle habe ich aber aus der Hinterleibsspitze ein Praeparat angefertigt und da dies in der Erscheinung etwas abweicht, gebe ich ron den Differenzen eine Skizze (Fig. 7). Die Penishaken sind gedrungener, das letzte Stermit ist stärker gewölbt und bildet am Ende einen in der Mitte etwas eingedrïckten abgerundeten Absatz; der Ausschnitt ist wesentlich kleiner. Falls sich dicse Form wirklich im Capland finden sollte, so dïrfte es sich $n$ m eine besondere dem Psocus quadrimaculatus Lark. sehr nahe stehende Art handeln. Vorläufig bleibt diese Frage unentschieden. 
Die mir privatim vorliegenden und grusstenteils von mir gesimmelten Stucke von Psocus quadrimaculatus LATk. (ơnd q) fige ich hier nuch \%am Vergleich an :

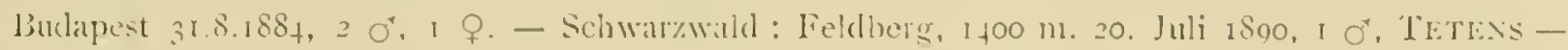

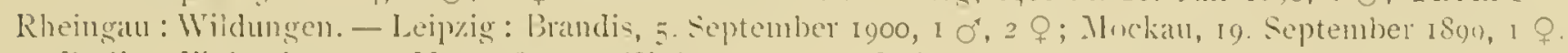

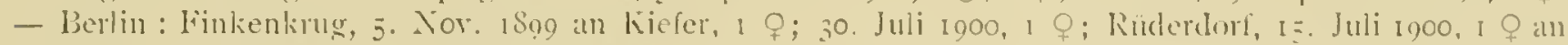
Sumbucus. - Sacheen : Tharand bei 1)resclen, 20.7.1000, 1 o an Kiefer. - Merlin: Spandian, 1.8.1900, I o an

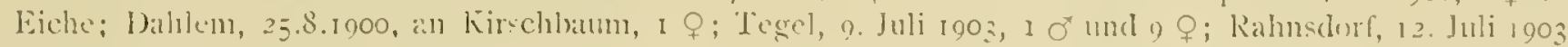
an Kieler, $\sigma^{x}$ und $q$ in Anzahl, 12. Juli 1003 an Eiche, or und $q$ in An\%hl, 12. Juli inoz an Erle, 3 o ;

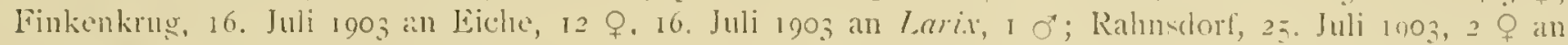
Sambucus, 25. Juli 1903 an Kiefer, I OT, 16 9, 25. Juli 1903 an Lindenstamm, 1 7, 26. Juli Ino3 an Eiche, I O', IS 9, $1+$ Larven und Nymphen, 26. Juli 1903 an Wachlolder, + 9 ; Tegel, 2. August 1903 an

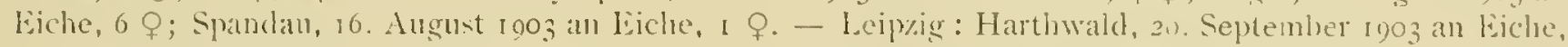

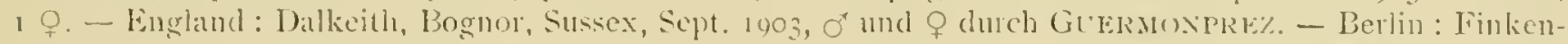

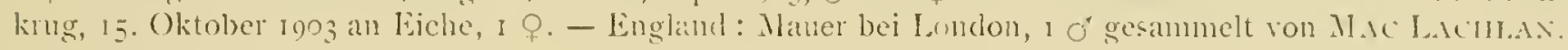

Letherwälntes Stuck, das ich durch TrTws erhalten habe, wurde von MAC LACHI.AN mit den Typen vom Psocus submebulosus STEPH. verglichen mo mit ihnen identificiert.

\section{Genus NEOPSOCUS Kiouri, ISS2.}

Neopsocus Kiolne, Ent. Nachr., S. Jhrrg., ISS2, p. 207 (ơ q).

Bimmole Navis, Mem. del primo Congreso de Naturalistas Espanoles. Maro igog, p. I57.

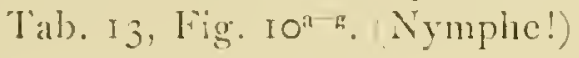

Neopsocus rhenanus KioLibe, iSS2.

Neopsocus rhenanus Kóne, Ent. Nachr., 8. Jhrg., i 882, p. 207 (O' o) Aug. I882).

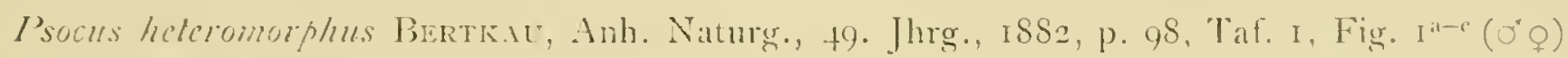
(Nor. 1882).

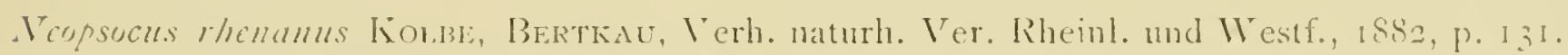

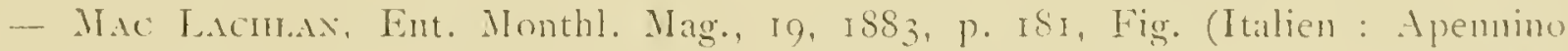
Pistojese). - Blirtkar, Sitzungsher. Niederrhcin. Ges. f. Niturk. und Heilkunde.

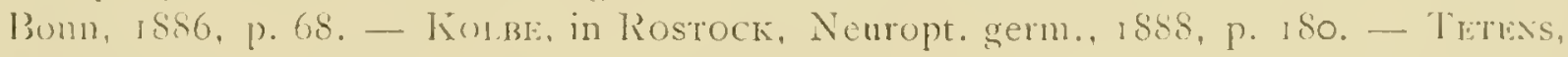

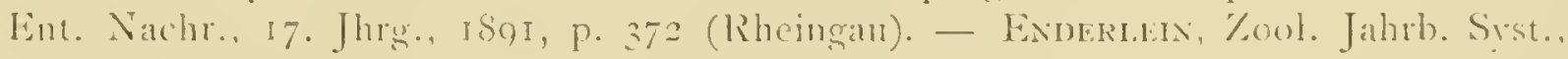
I +. I3d., I g00, p. I 47 .

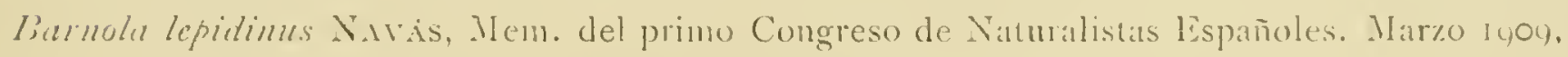
p. 157, 'Tab. I 3, ligg. 10:- (Nymphe!) (Spanien.)

Coll. Seits :

I O’, I 0 .

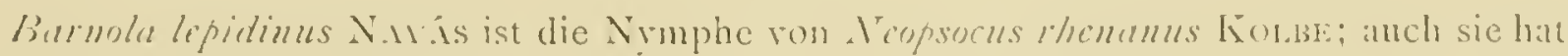
die eigenatigen geknöpften 1)rüsenhare, die anch das einzige (harateristicum bilden, die dic Imagines dieser fattung ron denen der Gattung Jsocus unterscheiden. 


\section{Genus AMPHIGERONTIA Kontis, isSo.}

Tipus : 4 . bifusciuta (LATR., I 799 ).

\section{Ampisigerontia bifasciata (L.1TR., I7y9).}

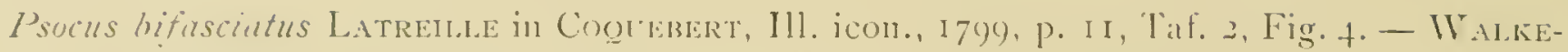

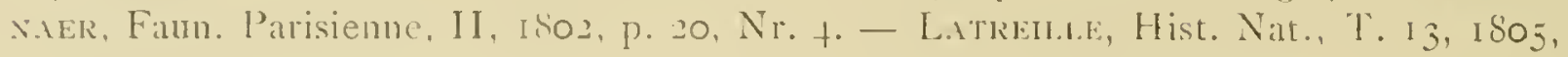
p. 72, Nr. 4. - Stephexs, Ill. Bril. Ent., Vol. 6, 18;6, p. 120.

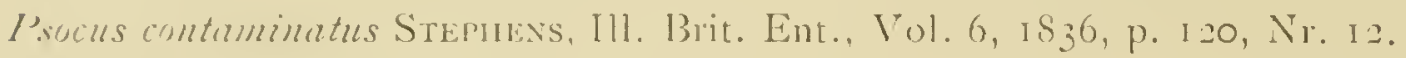

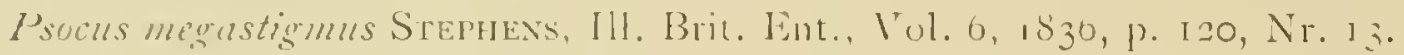
Pacus subfiscintus ZerTERstent, Ins. Lappon., is TO, p. 1052.

Procus quadrimaculatus Westwoon, Introd., II, I8fo, p. Io, Fig. 59, Nr. 9(2).

Procus bifusciutus Litr., HAgex, Terh. Zool. Bot. Ges. Wien, t866, p. 2 i 2. - Mac Lach-

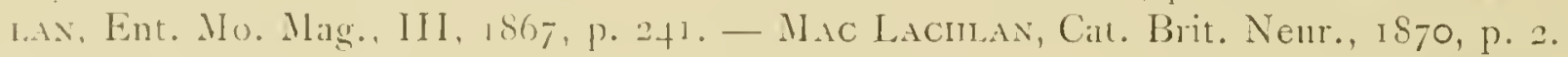
- Sulingerg, Vet. Ak. Förh., rs-S, Stockh., p. i6, Tal. i, Fig. S. - Kolbe, Mon. dentsch. Psoc., IsSo, p. IO4.

Amphigerontia bifascinta (L.1Tr.), Koure, Mon. deutsch. P'sociden, ISSO, p. Iof.

Etc., etc.

Coll. Setis :

1 f in der SELrs'schen Samunlung unter Ps. quadrimuculatus LATr., wohl die

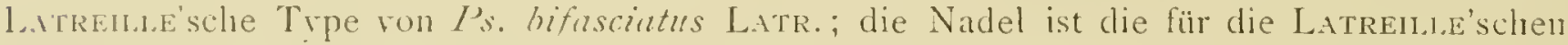
Stücke characteristische und mit sehr dickem, mregehmässigem Knopf die Länge der LATREHatéschen Nadeln ist sehr variabei).

$6 \%$ in der SErrs'schen Simmlung unter Ps. nehulosus STEPH.; ebenfalls wohl L.ATRtalas'sche 'Typen von I's. hifasciatus Latr.

Belgien: Spa (Lat Géronstère), 16. Oktober, 6 \%; Barisart, 24. Oktober, to Exemplare.

\section{Amphigerontia fasciata $(\mathrm{F} ., \mathrm{I}-87)$}

Hemerobius fiescintus Fabricus, Mant. Ins., i 787 . I, p. $247, \mathrm{Nr}$. I 3.

I'socus fusciatus F)., Fabricul's, Syst. Ent. Suppl., i 799, p. 203, Nr. t.

I'socus pilicumis Latrend.e in Coguenert, Ill, icon., 1799, p. Is, Taf. 2, Fig. 12. -

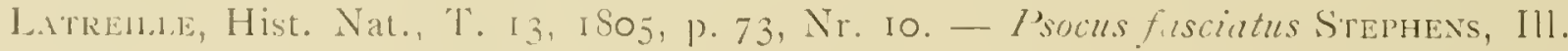
Brit. Ent., $1 \$ 30$, p. 118 , Nr. 13.

Ete., ete.

Coll. Sinas:

3 a af Latrembéschen Nadeln; ohne Angaben. Ein Exemplar trägt einen Zettel mit

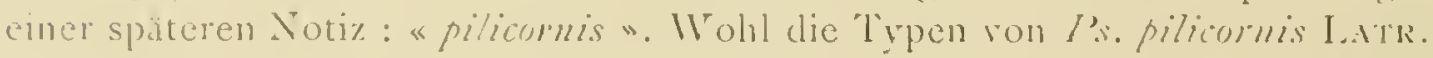

Belgien : Haren, 17. Juni, 1 Q; Longchamps bei Witremme, August, I q; Arlon, 1. Imni, I Q: Halloy bei ciner, t. und 5. Juni, = o. 


\section{Amphigerontia variegata (Li.TR., 1799).}

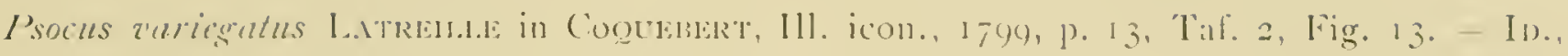

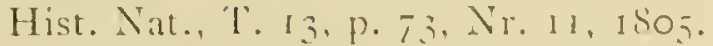

I'socus antumarius STrinexs, 111. Brit. Lint., 18:6, p. 118, Nro 15.

Psocus piciormis STtiphas, Ill. Brit. Ent., $1830,0.118, \mathrm{Nr} .2$.

Amphigeruntic z'ariegata (LATr.), Kon.36, Mon. dentsch. I'soc. 1850, p. 105.

Eitc., etc.

Coll. SEl, : :

Paris : I Exemplar. Defect, un I Vurder- und I Hinterflügel rorhanden (Coll.

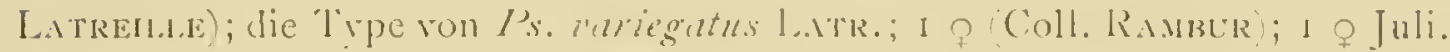

Belgien : Longchamps bei Waremme, 25. Juli, 1 o: 1 . Angust, $+q$; 20 . Angust, 1 .

\section{(icnuS TRICHADENOTECNUM IANIIRI.., 1909.}

Typus: T'. sexunitutum (L., 1-61). Europa.

Exdertens, Boll. Lab. Zool. Sc. Agr. I'ortici, III, 1009, p. 329.

\section{Trichadenotecnum sexpunctatum $(1, ., 1,61$.}

Hemerabius sexpunctatus Linvé, Fama Suec., Ed. II, 1761, p. 383.

Psucus sexpunctetus (L.), Fabricius, Suppl. Ent., 1798, p. 203, Nr. 5.

Trichadenotechum sexpunctutum (L.), Exneri.ers. Boll. Lab. Zool. Sc. Agr. l'ortici, III, 1909,

p. 329 .

Etc., etc.

Coll. Selis :

Belgien : 1. August, 1 .

\section{Trichadenotecnum majus (KolıE, Loens)}

I'socus mijor Kiol.rit.

Coll. Sistrs :

Belgien : Juli, I f: Longchamps bei Waremme, 25. Juli, 2 q; 12. Juli, 1 q: 2S. August, I $q$.

Genus Clematostigma ENHeri, 1906.

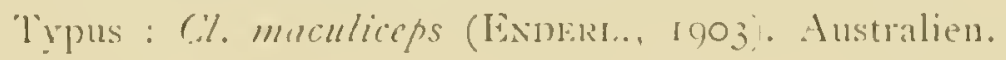

\section{Clematostigma morio (1.ATk., 1794).}

I'socus morio Litrlint, E, Bull. Soc. philom., I, r794, P. S5, Nr. 7. - In., in Coguebert,

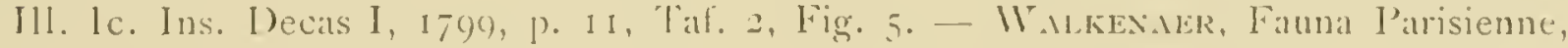


II, ISOz, p. 20, Nr. 5. - Burmeister, Handb. Eut., II, I839, p. 78i, Nr. 20. ..

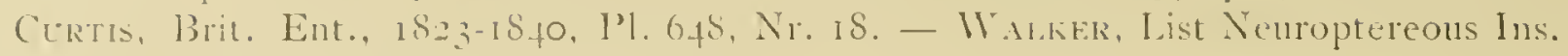
Brit. Mus., III, $1853,1 \%, 406$, Nr. 52.

Eti... eli.

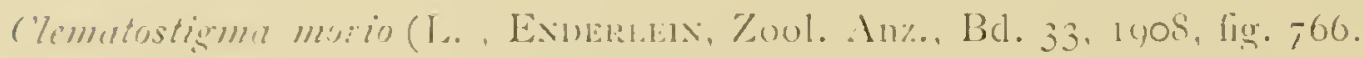

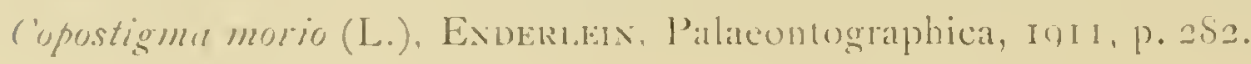

litte., ete.

Coll. Slitis :

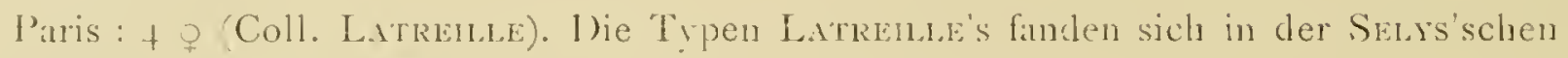
Summlung unter I's. qundrimaculatus L.ATr.

Madrid : 6. Juni. I Exemplar (\%erbrochen. In der SEn s'schen Sammlung und fraglich aus der Coll. Latrenthe.

Genus STENOPSOCUS HAG., is66.

Typus : St. immuculatus (STeph., is 36$)$. Emropa.

\section{Stenopsocus immaculatus (STEPH., I $S_{3} 6$ ).}

Psocus immaculatus Stephess, Ill. Brit. Ent., I 836, p. 12.5, Nr. 30.

I'socus rufescens STEpHExs, 111. Brit. Ent., i 836 , p. I25, Nr. 3 I.

I'sacus flarescens Stephexs, I11. Brit. Ent., 1836, p. I25. Nr. 32.

I'sucus renosus STephesis, Ill. Brit. Ent., r836, p. I 21, Nr. 17.

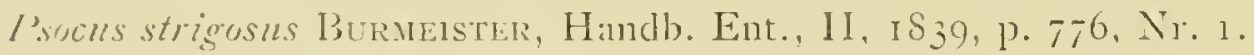

I'sucus fluzicans Zetrerstedt, Ins. Lappon., is fo, p. 1054, Nr. S.

l'socus subfumipennis Zetrerstedt, Ins. Lappon., I 8.40, p. I053, Nr. 5.

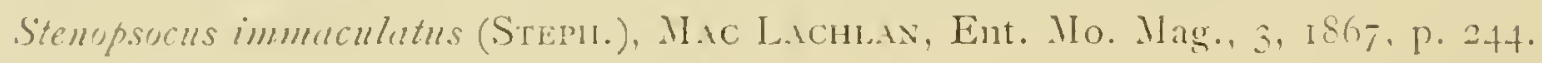

Eitc., etc.

Coll. Selis :

Paris : 1 o (CoH. LATREHLLE).

Belgien : Longchamps bei Waremme, JuIi, 1 q ; 23. Ingust, I Ex.; 24 . Angust, 3 Ex.; 25. August, I Ex. Halloy bei Ciney, 2S. August, I q; 5. September, z Ex.; if. September, I Ex.: 19. September, i $q$; 6. September, I q; Spa, 7. August, I Ex.; Tomelet (Spa), 17. ()ktoher, I Ex.

\section{Stenopsocus Lachlani lior.Bł.}

Conl serrs:

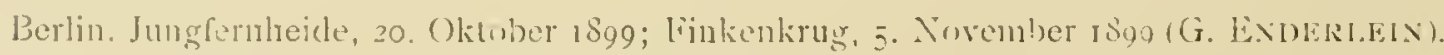


Stenopsocus stigmaticus (IMH. ('t L.,IBR., I $\delta_{f}(0)$.

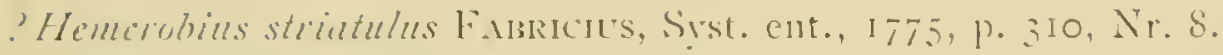

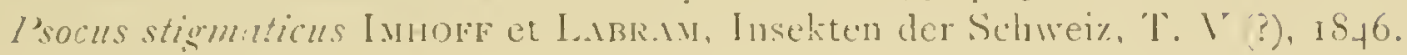

Eitic, etc.

(oil. Silis's:

Belgien : Longchimps hei Waremme, Juli, ; q; 20. Angust, I q; 25. Juni, 8 Lx.; Juli, I Ex.: 20. Ingust, 7 Ex.: 6. September, 1 Lx.; I. Oktober, 1 q Halloy bei Ciner.

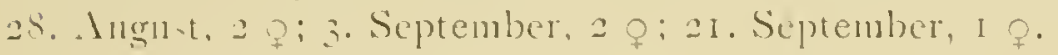

Genus GRAPHOPSOCUS Kon.BE, 1850.

Typus : G. crucintus (L., 1,68). Europil.

Graphopsocus cruciatus $(1 ., 1768)$.

Unm.M..., 1)issertal.. I $753, \mathrm{p}, 26, \mathrm{Nr} .51$.

Hemerob. us crncietus Lixí, Syst. Nat., Ed. XIII, 1708, T. 3, App., p. 225.

Hemerohius quadripunctutus Fabricu's, Mantissa Ins., 1. 1, 1787, p. $248, \mathrm{Nr} .17$.

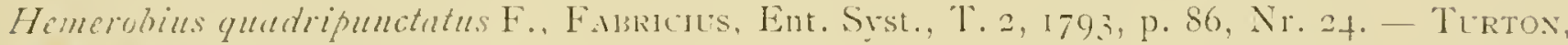

A generil srstem of mature, ISo6, p. +03 .

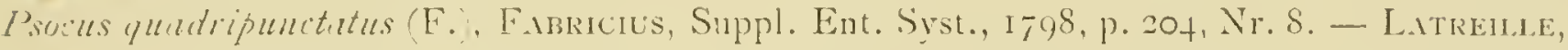
in Coglenert, 111. Icon., 1799, p. 12, Taf. 2, Fig. 9. - Stephens, 111. Brit. Ent.,

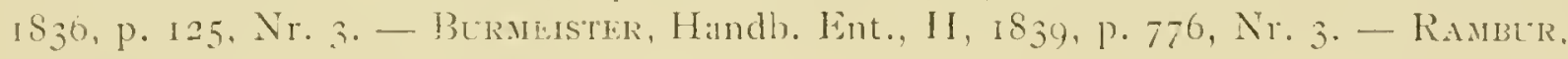

Hist. Neuropt., $18+2$, p. 321, Nr. 33 .

I'socus subocellutus Stepuexs, Ill. Brit. Ent., $1836, \mathrm{p} .124, \mathrm{Nr} .19$.

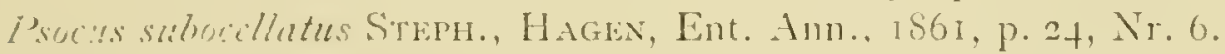

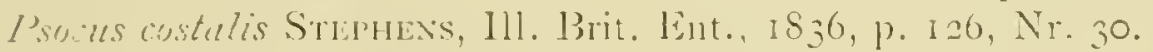

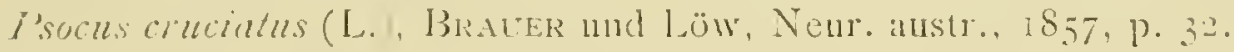

Sténopsocus crucietus (L.), Mac Lachina, Ent. Monthl. Milg., Vul. 3, 1867, p. 245. -

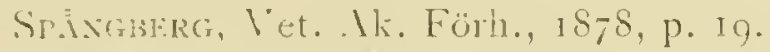

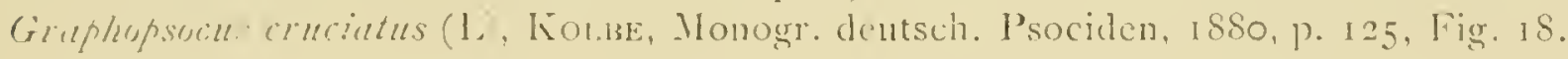

Eル, cに.

Coll. Sists:

1 f, anl kumer Latrandéschen Nadel; mit späteres Notiz : « quadripunctutus ",

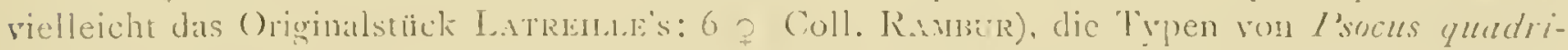
punctutus R.1.:2; 2. October, 1 Q: J11li, 15 \%.

Belgien : Spi, 16. Oktober, 1 2; Longchamps bei Warcmine, 25. Juli, I Ex.; r. Angust, 2 Ex; 6. Oktober, 7 Lx; 15. Oktober, I Ex. Malrisat, 24. ()kituber, 2 Ex. 


\title{
FAM. C A E C I L I I D A E.
}

\author{
Genis CAECILIUS CURT., I $\delta_{37}$.
}

Caecilius flavidus (Steri., $I \$ ; 6)$.

I'socus fluzidus Stephexs, Ill. Brit. Ent., i 836, p. 122, Nr. 20.

I'socus flaricaus Stephexs, Ill. Brit. Ent., I 836, p. I23, $\mathrm{Tr} .2 \mathrm{I}$.

I'socus ochroptorus Stephexs, Ill. Brit. Ent., I 836, p. I 22, N1. I9.

I’socus subpunctutus Stephiss, IH. Brit. Ent., i $S_{3} 6$, p. I $26, \mathrm{Nr} .34$.

Caccilus strigosus CurTis, Brit. Ent., I837, p. $648, \mathrm{~N} r .+8$.

Cinccilus flavidus nom. nud. Curris, $A$ guide to an arrangement of Brit. Ins., Ed. II, i $8_{37}$, p. I66, Nr. 19".

I'socus borë̈llus Zettrestedt, Ins. Lappon., is $8_{+0}$, p. 1053.

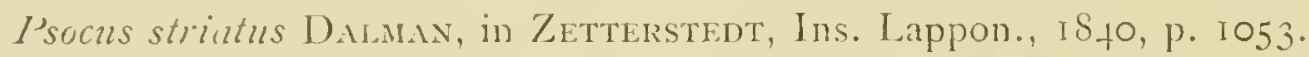

('accilius flaz'idus (Steph.), Mac Lachian, Ent. Mo. Mag., V'ol. 3, IS67, p. 27I.

Coll. Sel.ys :

Paris : 6 Ex. Coll. Rambur) (I'socus flazidus Steph., Rambur).

Belgien : Longchamps bei Waremme, 3 Ex.: Haut-Marais (Spa), I I. Juni, I o ; Longchamps bei Waremue, 5. August, I $q$; 20. August, I $q$; Juli 2 Ex.; Haut-Marais (Spa), I I. September, 3 Ex.; Halloy bei Ciney, 20. August, I Ex.; 6. September, 1 Ex.; 9. September, I Ex.; г 2 . September, z Ex.

\section{Caecilius obsoletus Sterh., I $\delta_{3}(6)$.}

I'socus obsolctus Srmeriexs, Ill. Brit. Ent., i 836, p. $123, \mathrm{Nr}_{2} 22$.

Etc., etc.

\section{Coll. Skils :}

Paris : 2 o Coll. Latreil..e) (ohme Namen-Etikette).

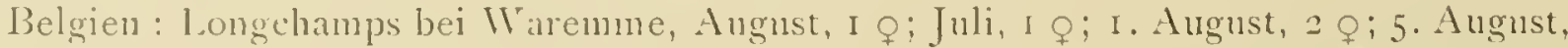
5 \&; I +. August, I ₹; 25 . Juli, I q 6. Oktober, I Ex.; I. Oktober, I Ex.; Haut-Marais (Spa), II. September, $S$ o; 15. September, I Ex.; Spa, 16. Oktoher, 3 Ix.; Hallor bei Ciney, 12. September, 2 Lx.; I 3. September, I Ex.; I 5. September, 2 Ex. 
Caecilius fuscopterus (LATR., I799).

Psocus fuscopterus Litrena.e in Cognemext, Ill. icon. Ins., I, I799, p. 10, Taf. 2, Fig. 2. Psocus vithlus 1)ndax, Analectal Ent., 1823, p. 5s, Nr. 13.

C'aecilius fenestratus Cukris, Brit. Bnt., is 37, p. $648, \mathrm{Nr}, 25$, Fig.

("uecilius fuscopterus (L.ATr.) Mac L.⿲chuax, Ent. Monthy Man., 2, I867, p. 272.

C'uccilius z'ittutus (1).u.n. Rostock, l'er. Naturk. Zwickan., 1877, p. 57.

Etc., etc.

Coll. Siriss :

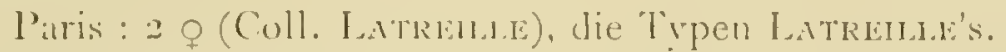

Belgien : Halloy bei Ciner, 16. September, 2 q; Hant-Marais Spa), I . September, i $\%$; Halloy bei Cines, 3. September, a Ex.; 9. September, + Ex.; ro. September, I Ex.; 24. August, + Ex.; 28. August, I IXx; fonnelet (Spa), г7. Oktober, I Ex.

\section{Caecilius piceus KoıßBE, ISS2.}

Caccilius picrus Kor.3e, Ent. Nachr., ISS2, Nr. 15. pp. 210, 211.

Etc., etc.

Coll. Seirs :

Belgien : 1 \%; Spa, 16. Oktoher, 1 .

Genus TRICHOPSOCUS Kolbe, is8s.

KulBe in Rosrock, Neur. germ., is88, p. 177.

Trpus : Tr. hirtcllus (M.ıc Lachian).

Trichopsocus Dalii (Mac Lachlax, I867).

(inccilius Malii Mac Lachux, Ent. Monthy Mag., V'ol. 3, I867, p. 272, Taf. 2, Fig. 6.

I q mit den Zettelangabe "immunis ".

Coll. Sirss :

Belgien : Jaleken bei Brïssel. In Palmenhäusern an Palmen. I q Vax Volxem.

Laclnesilla 11 istrw. I 8 .

Typus: L. pediculurie L. 1758 (Europa, cosmopolitisch verschleppt .

Fig. I und 2 .

Lachesilla Mrstwoon, Introd. modern. Classilic. of Insects, Vol. 2, 18,0, p. 19, Fig. 59: 16, 17 und 18 (aul p. IS ('). - Anhang dam : Symopsis of the Genera of British Insects,

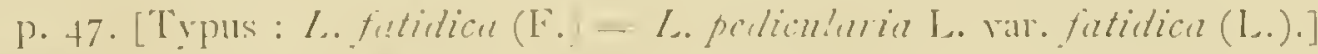

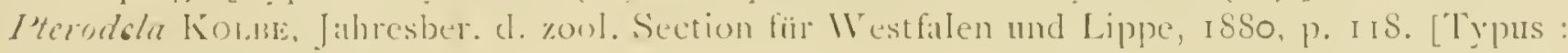
P. perticularin L.] 
Gomphocuccilius Eneren, Zuol. Jahresh. Syst., Vol. 14, 1900, p. 155, Talf. 9, Fig. 23. [Typus : Gr. trpetoides Exineri.., I000, Peru.] (Lmlasst Arten mit lang behanten Adern und Rand des Vorderflügels).

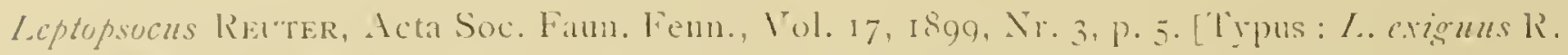

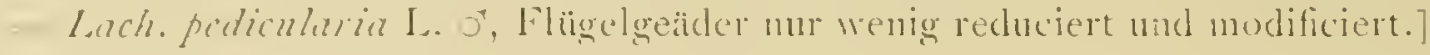

IIEsTWOON, loc, cit., P. t7:

"Head transierse; tarsi a jointed, wings o in 9,2 short in o ; legs slender. My lig. 59,16. »

1) ie WEstwoov'sche Gattungsdiagnose bezieht sich anf die mehr oder weniger kurz-

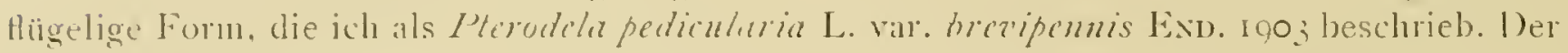
Name Lachesilla muss für Ptcrodela eintreten. Der einzige durchgreifende Interschied der rollgetlügelten Form ron Cacilius ist die Anwesenheit eines Klanenzahnes. Allerdings sind auch stets der Hinterflügeladern und -rand unbehart; im Vorderflïgel weisen zwar die emopäischen Formen nur mehr oder weniger Spuren ron Haaren auf, aber bei L. trypetoide's (ExDers.. 1900) aus Peru hat der Vorderflugel auf Rand und Adern lange Haare.

Die kurallügelige Form hat in männlichen Geschlecht (Fiğ. I und 2) häufig mur die Vorderflïgel entwickelt, die mehr oder weniger verkürzt sind und bei denen das Geäler je

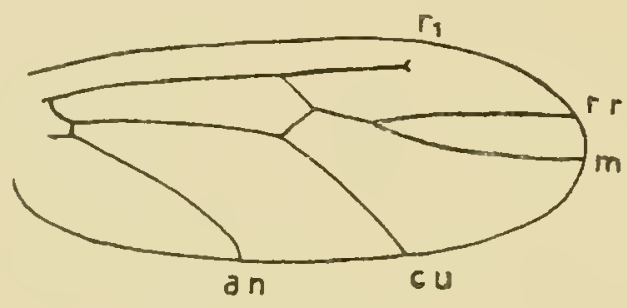

FIs. I.

Lachesilha pedicularia (L.) var. fatidica (L..) $\mathrm{O}^{r}$ Flügef (vergr. 160:1).

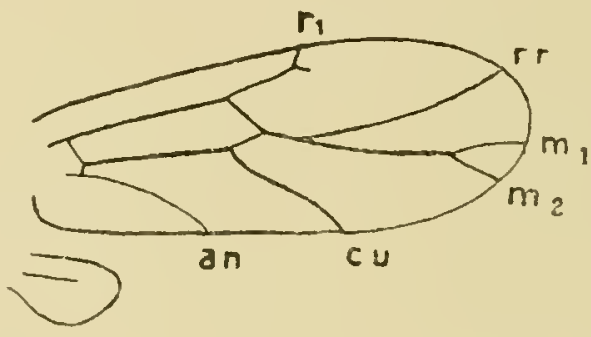

Fig. 2.

Luclucsilla proticularia (L.) var. fubidica (I.) O Flügel (vergr, 160:1).

nach Crad der Reduction ebenfalls reduciert ist. Der Hinterllägel fehlt entweder ganz, oder ist meist nur gan\% winzig entwickelt, so class nur noch die Spur einer Längsader rorhanden ist. 1) le ligur Wrstwoon's deckt sich ungefäh mit meiner Figur z. Beim weiblichen Geschlecht ist bei der kurzgetligelten Form meist der Vorderflügel nur in Form einer aderlosen Sichuppe, während der Hinterflïgel völlig fehlt.

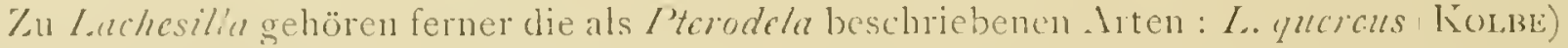

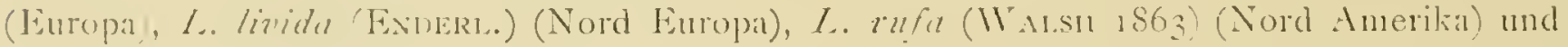
L. trupetoides (E,X)ER1.. I gO0) (Y'eru).

\section{Lachesilla pedicularia (L.., 1758 ).}

Hemerobius Haricuns Lixé, liam. Snec., Ed. 1, 1746, p. 223, Nr. 736.

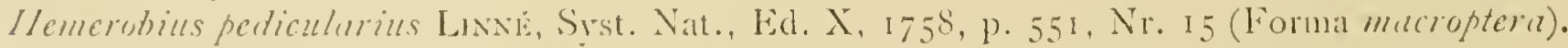

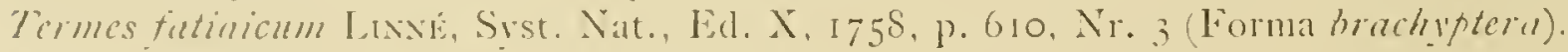

İemerobius flaricuns Lisé, Sist. Nat., Ed. X, 175S, p. 550, Nr. 10. 


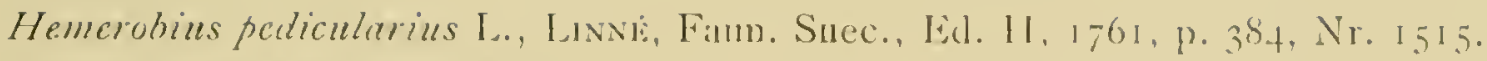
Termes fatidicum Linne, Faun. Suec., Ed. 11, 1701, p. 475, Nr. 1938.

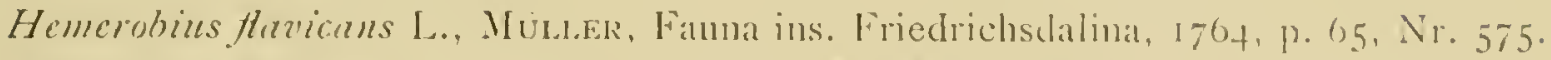

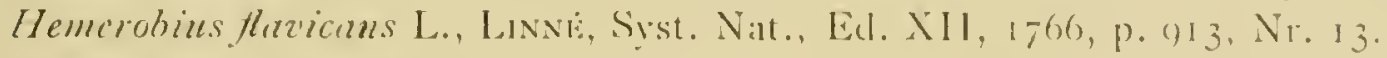

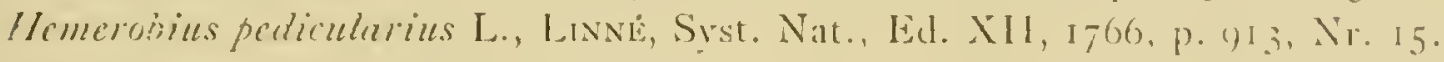

Termes futidicum L., Lindï, Sist. Nitt., Lid. XII, 1766, p. IO16, Nr. 3.

Hemerobius flavicans L., Fabkicus, Syst. Ent., 1775, p. 310, Nr. 10.

Hemerobius abdominalis FanRicus, Syst. Ent., 177.5. p. 310, Nr. 12.

llemerobius fatidicus L., Fankicus, Syst. Ent., 1775, p. 31 , N1. I

Memerubius flavicans L., Mulder, Zool. Dam. P'rodromus, I776, 1). 1 f6, Nr. 1683.

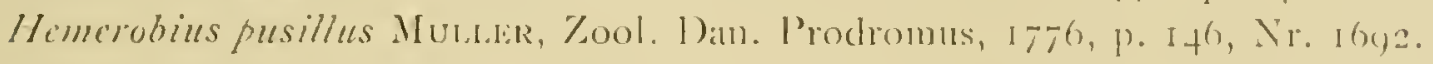

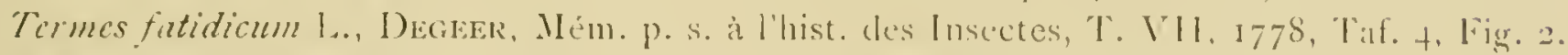

Ilemerobius abdominalis F., Fabscits, Reise nach Norwegen, 1779, p. 318.

Ilemerobius pedicularius L., Schkank, Enum. Ins., I7S1, p. 31 +, Nr.630.

Hemerohius tharicans L.., FanRIcils, Species Insect. 'T. 1, i781, p. 394.

Hemerabius abdominalis F., F.ABRacus, Species Insect., T. 1, 1781, p. 394, Nr. 14.

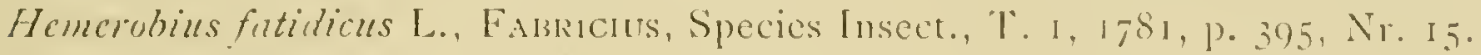

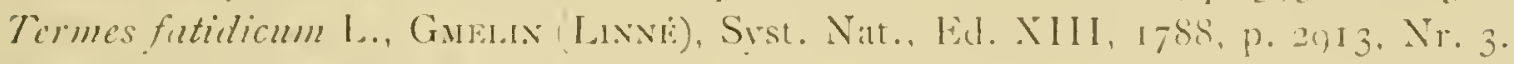

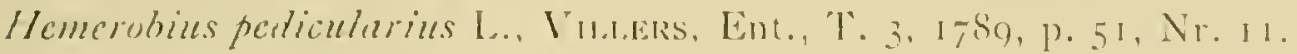

Hemerobius athominalis F., Fankicus, Ent. Sist., 1, 2. 1793, p. 86, Nr. 25.

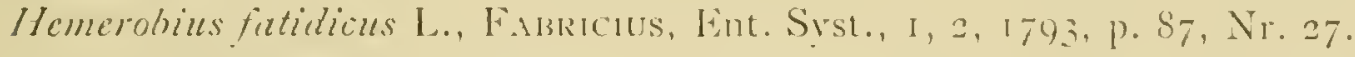

Psocus pedicularius L., Latren,te, Bull. Soc. Phitom., 'T. I, 1794, 1). 85, Nr. I.

Psucus abduminalis F., Fabrucius, Suppl. Ent. 1798, p. 204. Nr. 9.

I'sucus flazicans Fi., F.ubricus, Suppl. Ent., 1798, p. 203, Nr. 2.

Isocus futidicus L.., Fabricus, Suppl. Ent., 1798, p. 204, Nr. I I Forma brachyptera).

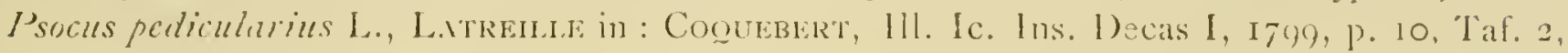
Fig. 1 .

Memerobius abduminalis F, Schraxk, Finn. Boicil, T. 2, 1802, p. 191, Nr. 1929.

llemerobius fittilicus L., Schraxk, Faun. Boica, 'T. 2, ISO2, p. 192, Nr. 193 I.

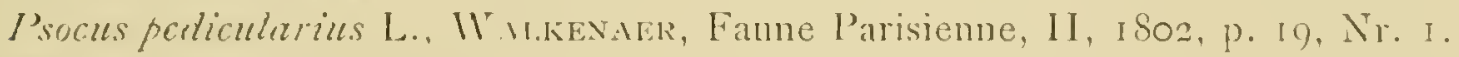

Hemerobius abdominalis F., 'luRTOx, A general Syst of nature, III, 1806, p. t03. - STIW.ART,

Elements of natural history, II, I8I7, P. 2I7.

Atropos fatidicus L., STerhess, Cat. Brit. Ins., I 829, p. 3I . N1. 2.

Atrupos fatidicus L., Stephens, Illustr. Brit. Ent, 1836, p. 129, Nr. 2.

Psocus nigricans SterheNs, Illustr. Brit. Ent., 1836, p. 127. Nr. fo.

Psocus dubius Strpuexs, Illustr. Brit. Ent., i\$36, p. 127, Nr. +2.

Atropos fatidicus L., CurTis, Brit. Ent., 1837 , p. I66, $\mathrm{Nr}_{\mathrm{r}}$ 2.

Psucus ubduminalis L., Clorts, Brit. Ent., 1\$37, p. 648, Nr. 24.

I'socus pulsatorius L., Zirterstiar, Ins. Lapp., I\$39, p. 1054.

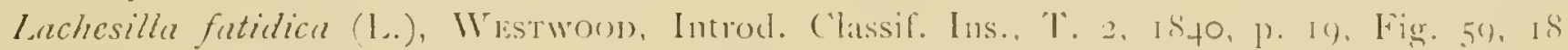

(Forma brechypterie).

I'socus hinotatus Ramistre, Nentopt., is f2, p. 324, Nir. 14.

Atropos fatidicus (L.), W.M.kik, (att. Nemupt. Brit. Mnscum, 1852-53, p. 500, Nr. 2. 
Psucus domesticus Burmeister, Handbuch Ent., i $832-1855$, p. 777 , Nr. 4. - Burmeister, BrAler, Neur. anstr., I 857 , p. 60 .

Psocus abdominalis F., Haciex, Entomologist's Annual, I861, p. 28, Nr. I 4 . Lachesis fatidica (L.), Hagri, Entomologist's Anmual, IS6I, p. 22, Nr. 3.

I'socus sulicis H.trex, Neur. N. America, I861, p. I3 (Nord-Amerikia).

I'socus gcologus W'x.su, Proc. Ac. Nat. sc. Phil., 1862, p. 362 (Nord-Amerika).

Lachesilh fatidica (L.), H.1Gex, Ent. Monthly Mag., II, 1865, p. I24, Nr. I.

Atropos fidtidicn L.), H.anex, Ent. Monthly Mag., II, i 865, p. 12 I, Nr. 4.

Psocus pediculurius L., Haciex, Stett. Ent. Zeit., I865, p. 229.

Atropos fattidica L., Hagw, Verh. Zool. Bot. Ges. Wien, r S66, p. 204.

Cuccilins pedicularius L., H.AGEx, Verh. Zool. Bot. Ges. Wien, I866, p. 206.

Elipsocus thricuns (L.), Hagex, Verl. Zool. Bot. Ges. Wien, r866, p. 207.

Lachesilla futidica L., HAGEN, Stett. Ent. Zeit., 1866, p. 238.

Ciucilius pediculurius L., Mac Lachlan, Ent. Monthly Mag., Vol. III, 1867, p. 17; Cat.

Brit. Neur., I870, p. 3.

Psocus pusillus Harris, Ent. Corr., I 869, p. 33 I (Nord-Amerikil).

Elipsocus flazicans (L.), Rosrock, Jahr. Ver. Naturk. Zwickau, 1878, p. 100.

Gacilius pedicularius L., Spangatera, Oefers. Vet. Ak. Förh., II, i878, p. 24. - Brauer,

Ent. Nachr. l'utbus, 1878, p. 87 .

C'accilius pedicularius L., Rostock, Jahıb. Ver. Natum. Zwickiu, 1879, p. 90.

Pterodelu pedicularin (L.), Kou,Be, Jahr. Zool. Sect. Westfalen und Lippe, I 880, p. S9 und I I 8 .

Cinecilius pedicularius (L.), HAGEN, Psyche, Vol. III, IS8I, p. 195 (Nord-Amerika).

Myporetes futidicus (L.), Hagen, Ent. Zeit. Stettin, 1883, P. 320.

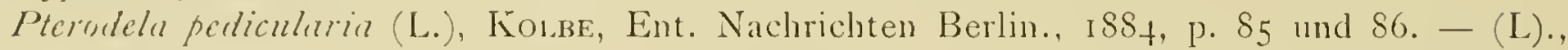

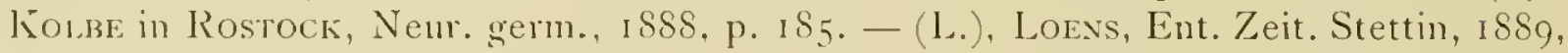

p. 331. - (L.), Tetens, Ent. Nachr., XVIl, is91, p. 372.-(L.), Reuter, Act. Faun.

Flor. Fennica, IX, I893, Nr. 4, p. 17. - (L.), Grard, Inn. Mag. Nat. Hist., Se1. 6,

Vol. I7, pp. I I I I 2 (Chile), 37 ; Act. Soc. scient. Chile, Vol. 1, 5, pp. 19-2 I.

Leptopsocus cxigums Reuter, Act. Soc. Famn. Flor. Fennica, XVII, 1899, p. 5 und 6 ( $\left.0^{7}\right)$.

Peroudela pedicularia (L.), Rnisaga, Riv. Pat. Veget., VIII, rgoo, p. 9 (sep.). - (L.) vitr.

Giardi Endertein, Zool. Jahrb. Syst., ił. 13d, 1901, p. 546, Taf. 35, Fig. io.

Leptupsucus crigums Reut., Enderiein, Ann. Mus. Nat. Hung., 1, 1903, p. 209; Zool. Anz.,

Bd 26, I 903, p. +3I (đ).

Pterudela pedicularia (L.), Enderienn, Zool. Jahrb. Syst., Vol. IS, 1903, p. 37t und 381

(Riigen, Schweden). - (L.), var. brezipennis Enderlein, Zool. Jahrb. Syst., Bd is,

1903 , p. 381 (Schweden, I entschland). - (L.), Schn, e, Sprawozdan Komisyi fiz. Al.

Umiej. w Krakowie, XXXVI, 1904, p. 37.

I.cptopsocus crigmus Reut, Reuter, Act. Soc. Fann. Flor. Fennica, Vol. 26, igot, Nr. 9, p. I8, Taf. II, Fig. 5-7 (०).

Pterodele pediculuria (L.), Dzienzifidurc\%, Sprawozdan Komisyi fiz. Ak. Umiej. w Krakowie, Vol. 38, igo5, p. i24 (Galizien). - (L.), Enderlein, Ent. \%eit. Stettin, Igo6, p. 3 I8; 28, Br. Westpr. Bot. Zool. Ver., igo6, p. 8I.-(L.), I) ZiEnziei.ewic\%,

Spraw. Kom. fiz. Ak. Umiej. w Krakowie, Vol. 42, I907, p. 24.

I'tcrudch pediculuris (L.), Banks, Cat. Neur. Ins., 1907, p. 7 (Nord-Ameriki). 
Pterodele pedicularin (L.), ENoERtits, Ent. Zeit. Stettin, 1907, p. 98 (Japan). - (L.), NAWAS, Brotéria, Ser. Zoot., I907, p. 282 (Spanien). - (L.), Tert.gkla, Ark. f. \%ool., Bd 5. Nr. S, I909, p. 9. - (L.), Exuleries in Voltzkow, Reise in Ost-Afrika, II, Igos, p. 250 (Comoren). - (L.), In., ibid., p. $2+6$ (Kamanische Insehn). - (L.), Reurer, Medd. Soc. Fanm. Flor. Fennici, Vol. 35, 1y09, p. 204. - (L.), vor Rosex, Schr. Phys. öl:on. Ges. Königsberg l’r., 5 I Jhrg. 1910, p. 335. - L.), var. breqipennis Exin., rox Rosex, Schr. Phrss. ökon. Ges. Königsberg Pr., 51. Jhrg, rg10, p. 335. - (L.), Okisoto, Anm. Mus. Nat. Hung., VIII, Ig10, p. 196 (Japan). - (L.), Navis. Bol. Soc. Aragonesa del Cienc. Natural., rq I fig. zio (Spanien).

Coll. Seriys :

Paris: I o (Coll. Latremble) (ohne Namenamgabe); I q (Coll. Ranbur) die Type von Ps. binotatus RAMBUR.

Belgien : Longchanps bei Waremme, ro. September, I q; 21. September, I q; liegre, 10. September, 10 Ex.; 16. September, 2 \%; Hallog bei Ciney, I q; 5 . September, 3 q; Himt Marais (Spa), 2 \%; I. September, 4 \%.

Lachesilla pedicularia (L.) var. brevipennis ExDERI.., I903.

Diese kur\%geflugehe finm steht \%wiscinen der macropteren form und h. peit. var. fatidica (L.). Vorder- und Hinterllïgel sind bei s und o etwas verkürzt und das Geäider ein wenig reduciert. Die ah. Giardi ExDeks. 1909 ist besser unbenannt zu lassen, sie stellt den Beginn der Geäderreduction dar.

\section{Lachesilla pedicularia (L.) var. fatidica (L.., I 758 ).}

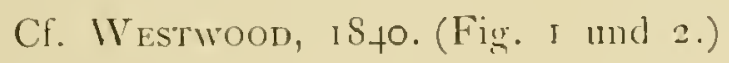

Diese extreme Form ist etwas kleiner und die Flügel des $q$ sind röllig reduciert, während beim o der Hintertlïgel nur als winziger. Stummel rorhanden ist oder ganz fehlt. l)er Vorderfligel des $\sigma$ ist dagegen vorhanden aber mehr oder weniger stark reduciert, so lang oder etwas kürzer als dits A bdomen. 1)as Geäder (Fig. I und 2) ist mehr oder weniger stark reduciert. Die obere der beiden angegebenen Figuren (Fig. I) stellt das Stadium der Geäderreduction, die ungeführ der Westwoon'schen Figu 59, is entspricht. $r$ ist ungegabelt; die Media ungegabelt oder mit kurzer Gabel, cu ist ungegabelt; die Axillaris ist reduciert, ebenso wie ein deutlich erkennbares P’terostigma fehlt. Der für die Gattung so characteristische Klanenzahn (bei ('aecilius und Verwandten fehlt er stets) ist rorhanden, zuweilen wird er aber klein und stumpf, so dass er etwas undeutlich erscheint.

Diese Form kommt hier und da in Häusern vor, besonders in (Oktober; vielleicht stellt sic eine Herbstform dar.

\section{Lachesilla quercus (Kon.131: I852).}

Pterodela quercus. Korosi:

('oll. Siriss :

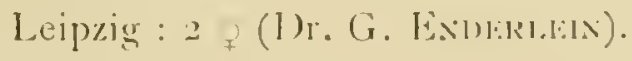




\section{Genus PERIPSOCUS H.in., I 866.}

\section{Peripsocus phaeopterus (STrPH., I $8_{3} 6$ ).}

Psocus phucupterus Stripuens, 111. Brit. Ent., I 836, p. 127, Nr. 39.

Psocus nigricormis Stephexs, Il]. Brit. Ent., I 836, p. 126, Nr. 38.

Cincilius fuscupterus CurTus, Guide, 2. Ed., 1837, 1) 166, Nr. 22.

('nccilius nigricomis CurTs, Guide, 2. Ed., I 837, p. 166, Nr. I 2 ".

l'socus obscurus R.smber, Hist. Nat. Neur., I 842, p. 322, Nr. го.

I'socus luricis Brimu, H.tglix, Stett. Ent. Zeit., 26, 1865, p. 229.

l'eripsocus phucopterus (Stepu.), Haciex, Verh. Zool. Bot. Ges. Wien, I6, I866, p. z Io.

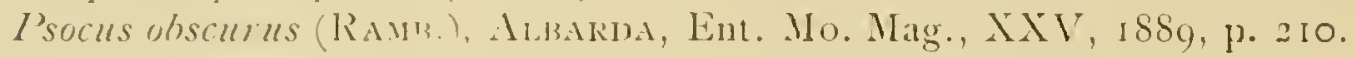

Etc, ete.

Coll. Sillys :

(Paris) : 2 q (Coll. Rambur) die Trpen Ramisur's.

Belgien : Longchamps bei lliaremme, Juli, 2 q; 25. Juli, I $q$; I. August, I q; Halloy bei Ciney, 6. Juli, 1 q.

\section{Peripsocus subfasciatus (RАMв., I $\delta_{4}$ ).}

P'sucus subfusciutus Rambur, Hist. Nat. Neuropt., I $\delta_{+2}$, p. 322.

I'cripsocus alboguttutus Data., Mac Lachlan, Ent. Mo. Mag., Vol. 3, I867, p. 273, Tal. 2,

Fig. 8. - 1).ı.M., 1D., Neur. Brit., 1870, p. 3.

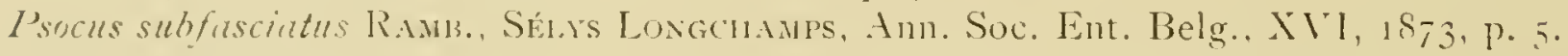

Peripsocus alboguthtus Sringrerg, P'soc. Suec. et Fenn., is 88 , p. 27, Fig. 17. - Malm.,

Kor.re, Monogr. deutschen Psoc., I880, p. I30, Fig. 20. - D.ı..., Id., Ent. Nachr., I882, Nr. 15, p. 212.

Peripsocus subpupillatus Mac Lacilan, Ent. Mo. Mag., I9, I883, p. 183. - Mc Lachu.,

Rosrock, Neur. germ., is 88, p. 171. - Mc Lichl., Kol.ze in Rostock, Neur germ.,

1888, p. 187. - Mc Lachl., Loens, Stett. Eut. Zeit., 1889 , p. 331. - Mc Lacill..,

Tetexs, Ent. Nachr., I 891, p. $372(p \cdot 4)$.

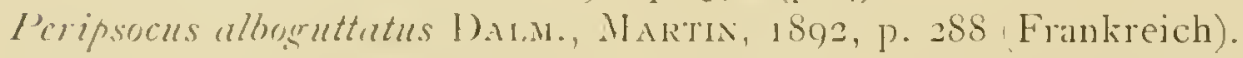

l'eripsocus subpupillatus Me Lacht.., Regni Soc. Scient Nat. Hungaria. Fauna Regni Hung.,

1 (y)o, p. 26.

Etc., etc.

Coll. SHils's :

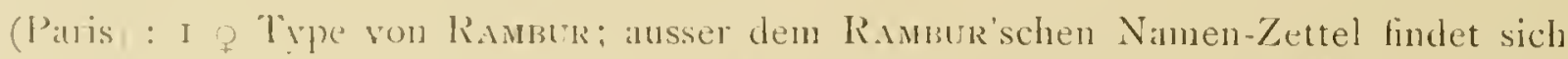
noch ein späterer Zettel mit der Bemerkung : «étiqueté subpupillutus» par MAC LachiaN en 1883 ».

Nach der Type ergicbt sich demuach die Zugehörigkeit von P. subpupillatus Mc Lacu.. zu I. subfisciatus (RAMusUR). 


\section{Peripsocus alboguttatus (1) $\wedge, \ldots, 1823$ ).}

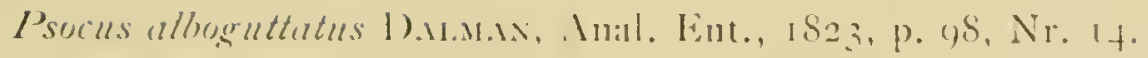

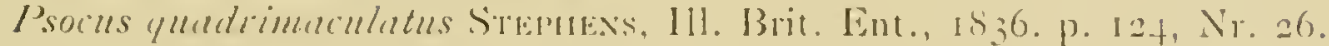

Psocus subfusciatus R.umber, Hist. Nat. Neur, 18f2, p. 322, Nr. 10.

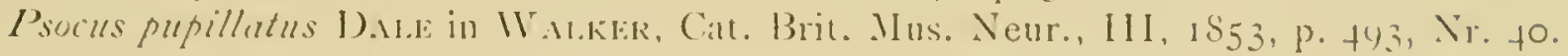

Eiti, ete.

Coll. SELSS :

1 q. mit einem 7ettel "pupillutus" und mit einem \%weiten Zettel walhognttatus " M. L.ACHI...X 1883 .

Coll. Sel,s

Belgien : Longchamps bei Wamemme, 1. August, 1 q, 12. Angust, 2 .

Genus BERTKAUIA ḰOI,BE, 1882.

Berthamin Kon,131, Ent. Nachr., I882, Nr. 15, p. 208 (o).

Lapithes Brokkat, Arch. für Naturgesch., t9. Jhrg, 1882, p. 100 (Q), Taf. I, Fig. 3 a-d.

Berthamia Enderteix, 2S. Ber. Westpreuss. Bot. Zool. Ver., 19o6, p. 73 (ơ).

Bertkauia lucifuga (R.1Mı, $1 \delta_{+2}$ ).

(Fig. 3, t. 5; Tafel 11, Fig. S).

Psucus lucifusus R.smber, Hist. Nat. Nemopt., $1 \AA_{f 2}$, p. $32+(q)$.

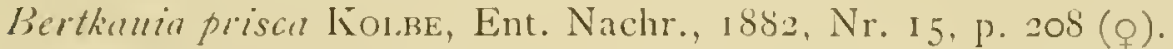

Lapithes pulicarius Bertiau, Areh. für Naturgesch., t9. Jhrg, 1882, p. 100, Taf. 1, Fig. 3 (a-d).

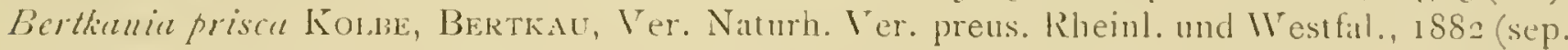

p. 3 und 8) (q). - Kol.be, Kor be in Rostuck, Neur.germ., i888, p. $188(q)$ - Kol.131,

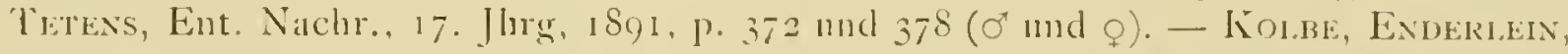

Zool. Jahrb. Syst., 1 +. B3, Igo1, p. 539 (q). - Kor.BE, In., Amu. Mus. Natt. Hung.,

Bd I, 1903, p. 20t (o" und o). - Kol.13E, In., Zool. An\%, 27. Bd, 1903, p. 133.

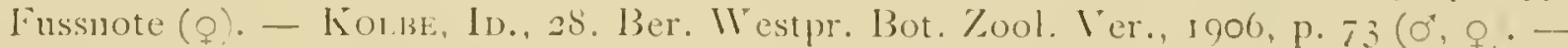



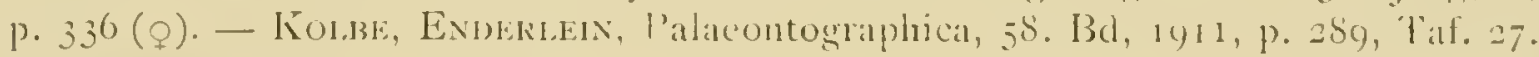

Berteania lucifuga (Ranis.).

Coll. Seriss.

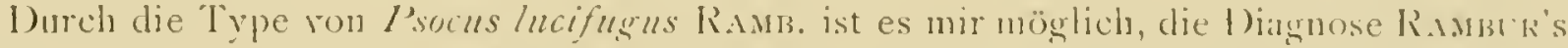
zu denten, die immernin das Tier erkennen läst. Die biologiscinen Notizen jedoch lassen es mit Bestimutheit denten. Diese Art lebt meist an altem Hol\%, sowohl in Kellern. Höhlen, Freibhäusern, als auclı in feuchteren Wädern unter abgebrochenen Aesten.

In Wäldern fand ich diese Art unter. Arten meist in Ciemeinschaft mit der Collembole

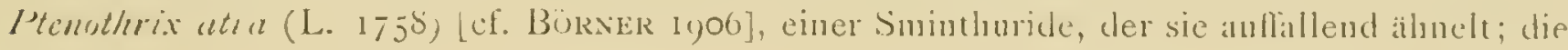


ausserordentlich langen spitzen Gonopoden des 9. Segmentes (Pl. II, Fig. S gp p) täuschen sogar die Springgabeln dieses Springschwanzes ror. Eine Besonderheit hat der Springschwan\% dadurch, dass die Fuhlerspit\%e schneeweiss ist.

Merkwürdiger Weise befand sich nun neben einem der auf Kartonblättchen anfgeklebten z Typen von R.umber ebenfalls ein Exemplar von I'tonothix atra (1. 1758).

lch weichte dieses Tier mit besonderer Sorgfalt auf, mon diese Tatsache festzustellen, da es mir kein Zufall \%u sein scheint.

Es dürfte tatsäichlich bei Berthanur lucifuga (Ramb. I $S_{+2}$ ) eine minetische Anpassung an Ptenothrix atra (L., 1758 ) vorhanden sein. Welchen Grund dieselbe hat, ist allerdings bisher nicht festznstellen gewesen.

1)as $0^{\prime}$ ist im Gegensat\% zum o geflügelt. Es ist bisher nur I O" erbentet worden, und жwar von TEtras (cf. l.c.). Nach diesen Stiicke, das sich im Berliner Zoologischen Museum befindet, habe ich die folgende Beschreibung des Ceäders und Abbildung (Fig. 3 angefertigt.

Geäder wie bei der Gattung Epipsocus H.AG., nur findet sich жwischen P'terostigma nud Radialranns ein Querast, der anf dem einen Flügel den Radialgabelstiel triflt (vergl Fig. 3), anf dem anderen in $r_{z+3}$ mündet. Die Areola postica ist sehr breit und gleichmässig flach gewölbt. Radialramus und Media im Vorderflïgel durch eine lange Querader verbunden, im Hintertlïgel eine kurze Strecke verschmolzen. Rand des Vorder- und Hinterfliigel behatart. Adern des Vorderflügels ohne die Analis pubesciert. Radialramus und Media im Hinterflügel pubesciert.

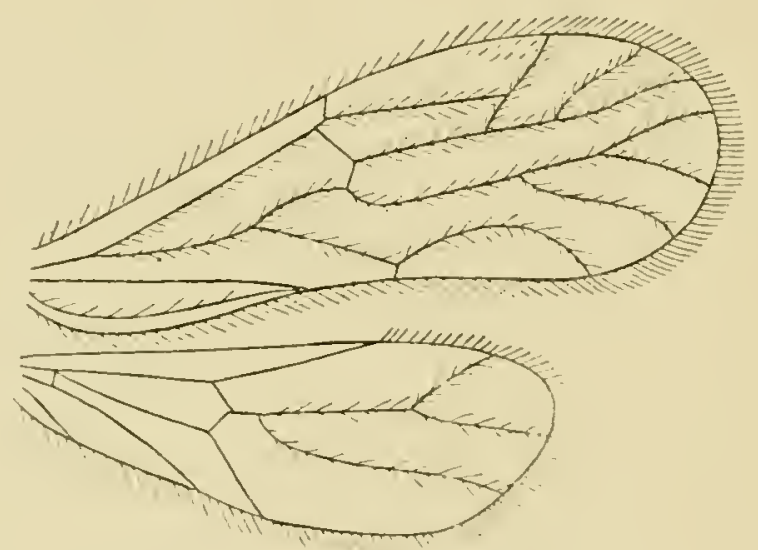

F19. 3. - Ierlanuin lucifuga Rasib. $\sigma^{x}$ Geäder (vergr 16:1).

Nach der Trpe Ramm's habe ich folgende Beschreibung und Abbildung der weiblichen (ienitalinhinge angefertigt.

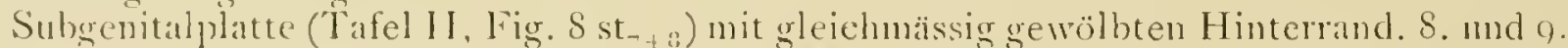
Tergit (tg: und tg ) ziemlich stark verschmolzen. Wittlere Telsonklappe (dte) ziemlich klein und dreieckig. Seitliche Telsonklappen grösser und breit dreieckig (Fig. S he). Gonopode des 8. Segmentes (Tafel II, Fig. S gp ) mässig lang, stilletförmig, die Spitze etwas abgeset»t und schr dün und spit\%. Gonopode des 9 . Segmentes (Tafel II, Fig. S (qp p) mit ansser ordentlich langem stilettförmigen Juhang (Medial-Gonopode); die lateralgonopode ist der verbreiterte Basalteil, der bei dieser Form relativ wenig abgeset»t und specificiert ist.

Die beiden inneren Laden der untersuchten Rambur'schen Type sind etwas verschieden. I) ie der rechten Seite (Fig. f) hat am mehr oder weniger schräg abgestut\%ten Ende cal. 7-8 Zähne, der innerste an kräftigsten, die linke ca. 2-3 mehr. 
Die inneren Laden der Naxilte sind relativ breit und reschmälern ich nach der Basis alhuählich stark.

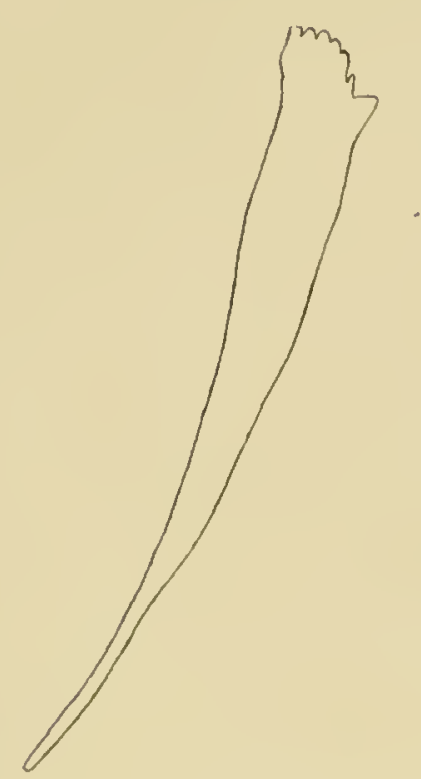

Firs. 4. - Bertkintial lucifugat Rasb. ? innere Lade der rechten Maxille ron unten. (Museum 13ruxelles) r60: 1 .

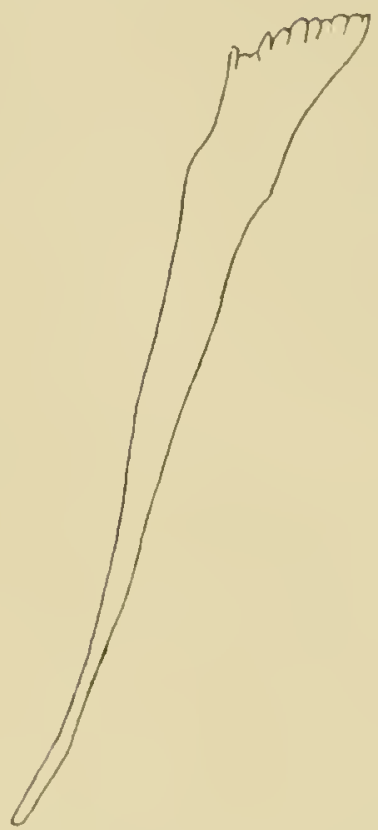

litrs, 5. - Berthatuh lucifuga Rash. Q Innere Lade der linke Maxille von unten. (Museum Bruxelles) 160: 1.

Die Original-I)iagnose ron :

Psocus lucifugus R.mubtr, Hist. Nat. Nemopt., 18 42 , fig. 324.

Loc. cit. ist :

"Plus du double phus gros que le binutatus ( ${ }^{2}$ ). légèrement velu: d'un brun-roux. T'ête, anus et base des cuisses d'un janne phus on moins obscur; antennes velues, plus longues que le corps. Complè'ement aptère (est-ce un insecte parfait ?). 'Tarses de deux articles.

Se troure sur le vieux bois des cares, qu'il parât ronger; marchand et comrant peu, mais santant asse\% vivement quand on le tonche $\left(^{(3)}\right.$. Il est très mou pendant la vie et son abdomen est très épais : le moindre attonchement suffit pour l'écräser. »

\section{Genus REUTERELLA ENIER1., I9O3.}

Typus R. hchimicula (ENuERı., IgO I) Europa.

Renterella Findertix, Zool. An\%, Bd 27, r903, p. 132 (q). - Reuter, Act. Soc. Fam.

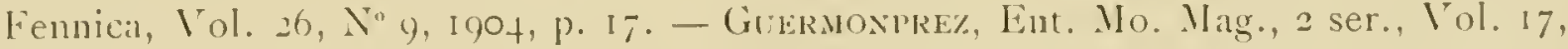

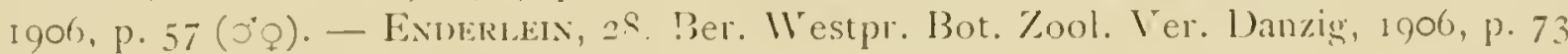
(o*o). - In., Palateontographica, Vol. $5^{8}, 19$ I , p. 289 .

(') = Iachesilla pedicularin (L.).

(2) Dies a Springen bezicht sich vermullich auf die sininthuride: Plenothrix atra (I. 1758), vergl. Pl. 21 und 22. B. lucifuga RAMB. springt nicht. 
Reuterella helvimacula (ENI)ERI.., lyor).

(Til. Il, Fig. 9).

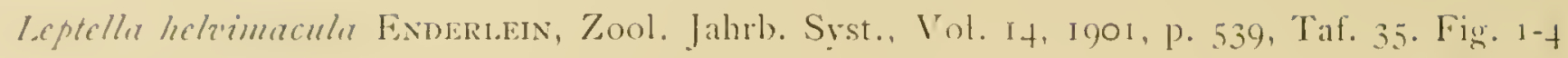
(q) (Berlin).

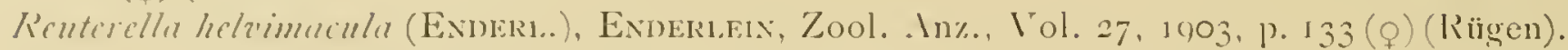

- (Exidert., Rertik, Act. Soc. Fann. Flor. Fennica, Vol, 26, N" 9, igot, p. i6,

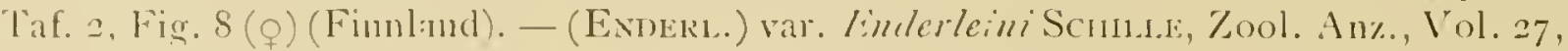
1904, p. 475 (q) (Galizien). - (EN1)ERน..), In., Splawozdả Komisvi li\%gograličnej Akad. Umiejetnósci w Krakowie, Íot. 38, 1904. p. 37 (q). - (Enneri..), Guermonprez. Ent. Mo. Mag., Ser. 2, Vol. I7, 1906, p. 57 (ơ), Taf. I, Fig. 1-I3 (England). -

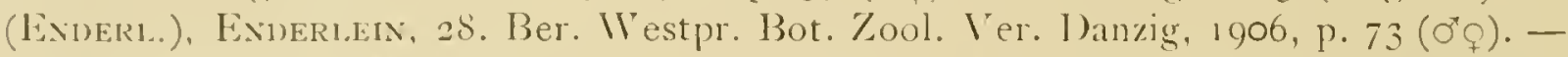
(Exderı, ), Reuter, Medd. Soc. Fam. Flor. Fennica, Vol.35, i go9, p. 20S. - (Exinfri..), rox Rosex, Schr. Ges. Königsberg, Vol. 51, I910, p. 336 (o) (Ost-Prenssen). (Exderi..), Enderiein, l'alaeontographica, Vol. 58, 1911, p. 289.

Die Subgenitalplatte des $q$ (Taf. II, Fig. 9) st $_{7+8}$ ) besteht aus den völlig verschmolzenen 7. und S. Sterniten; der Hintermand ist convex, in der Mitte stärker nach hinten ausgezogen mnd in a Spitzen endigend, die durch einen halbkreisförmigen Ausschnitt von einander getrennt sind; auf jeder Spitze zwe! Bürstchen. Gonopode des S. Segmentes (Taf. II, Fig. a gp.s) ziemlich lang, stilettförmig, alluählich zugespitzt. Mediangonopode des 9. Segmentes (mgpa) etwas kïrzer als die Gonopode des 8 . Segmentes, in Stilettform allmählich zugespitzt; bis zur Mitte etwals verdickt, dinn plötzlich dünn. Lateralgonopode des 9. Segmentes (Fig. 9 lgp g) gross und fast dreieckig. Mittlere Telsonklappe ('Tal. II, Fig. 9 dte) dreieckig, seitliche Telsonklappen (lte) ziemlich breit und kur\%; olme Sinnesfeld oder Sinnesorgan.

Das geflügelte o wurde ron Giermosprez beschrieben.

Coll. SEI.YS.

Rügen : 2 q gresammelt ron Dr. G. ENmlertein. 


\title{
SUBORDO : HETEROTECNOMERA. \\ CRYPTODERATA. \\ DERMOSTIGMATOPHORA.
}

\section{FAM. MESOPSOCIDAE.}

\author{
Genus LEPTODELLA Rien,. I90 .
}

Leptella Retrer, Act. Soc. Fann. Flor. Fennica, IX, N" t, 1893, p. t5, Fig. S.

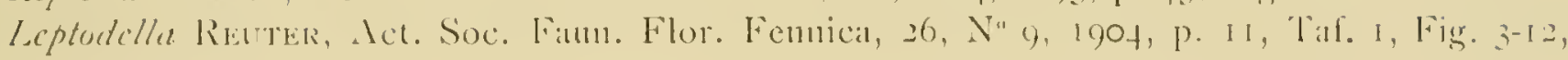

Tal. 2, Fig. 1.

\section{[Leptodella fusciceps (Reu־., I\$93) ('Taf. I1, Fig. 10, 11 ) Fig. 1), 7.]}

Leptella fusciceps Revres, . Act. Soc. Faun. Flor. Fennica, 9, $\mathrm{N}^{0}+1893, \mathrm{p}$. fh (7); pp. 17, 37.

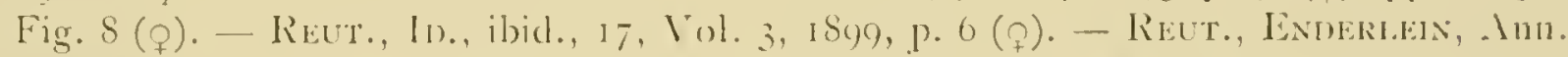
Mus. Nat. Hung., I, 1903, p. 204 ( ) . - Reut., In., \%ool. An\%, Bd 27, I903, p. I33 and i $3+(0 \%)$.

Leptodelle fusciceps (Retr.), Rermik, Act. Soc. Faun. Flor. Fennica, 26, No 6, 1904, p. 13,

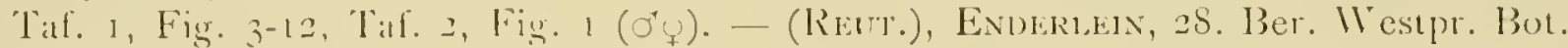
Zool. Ver., 1900, p. 7ł. - (Risur.), In., Palacontographica, Vol. 58, 1911, p. 290.

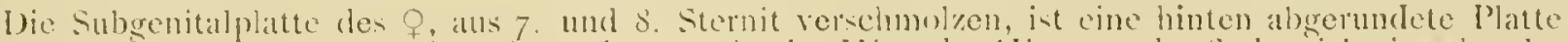

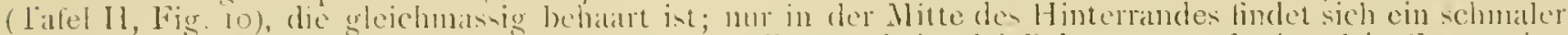

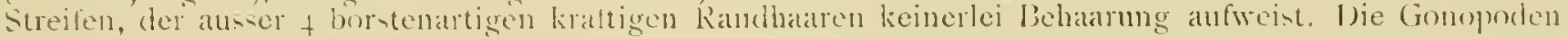
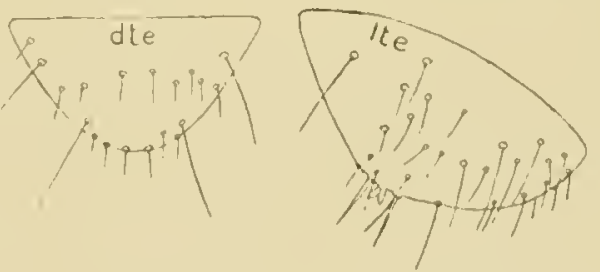

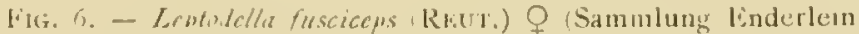
Fine Iaterale l'elsonklappel (lte) und die dorsale "lelsunklappel (dic). Vergr. $160: 1$.

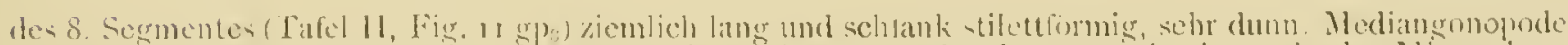

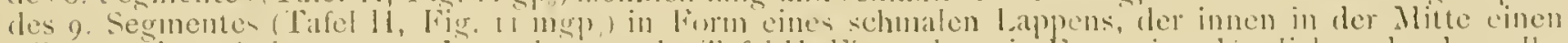

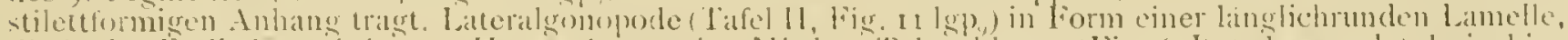

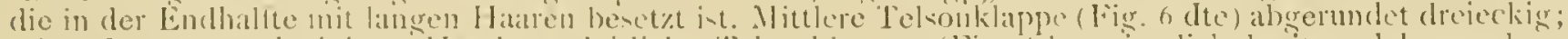

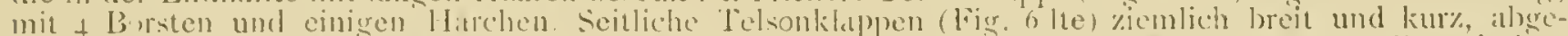

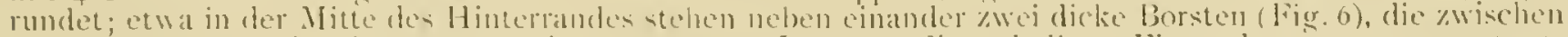

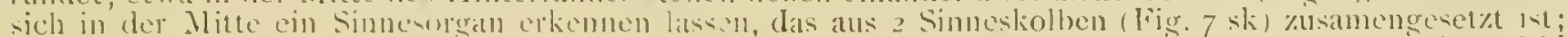

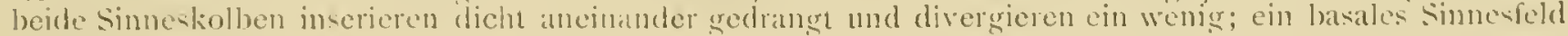
fehlt hier cbenfalls. 


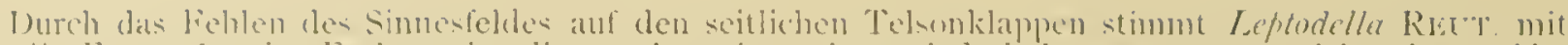

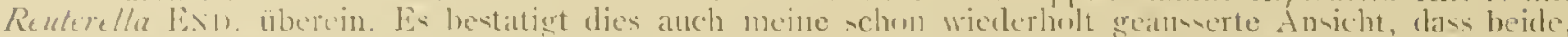

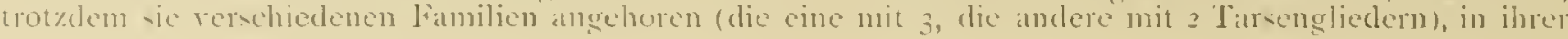

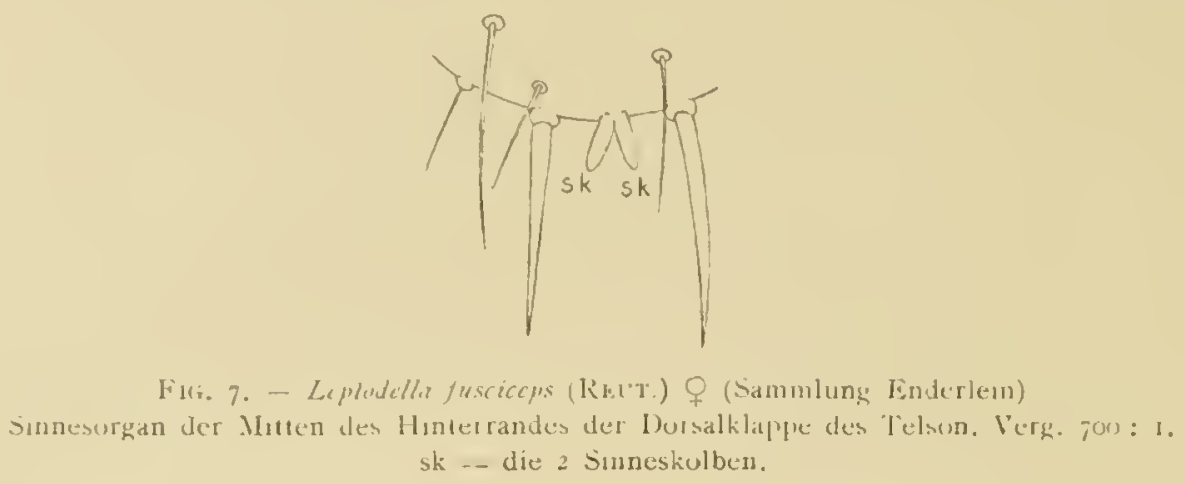

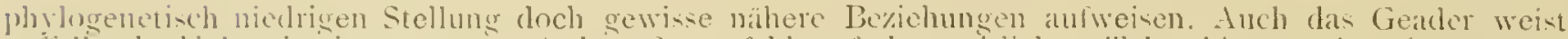

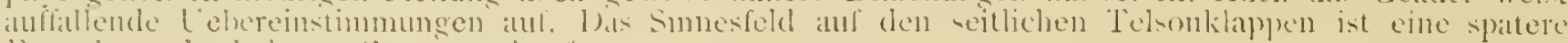
linwerhung der hoheren Copengnathentormen.

Genus MESOPSOCUS Kionki, is so.

\section{Mesopsocus unipunctatus (MULI. I $76+$ ).}

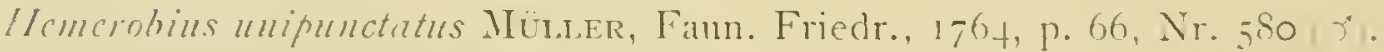

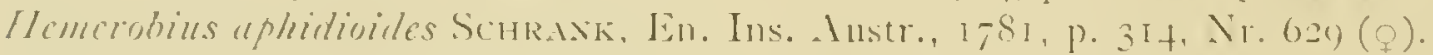

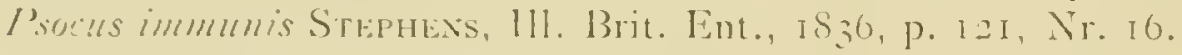

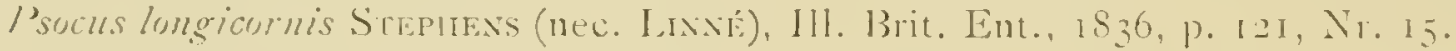

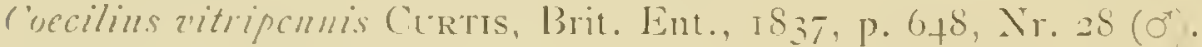

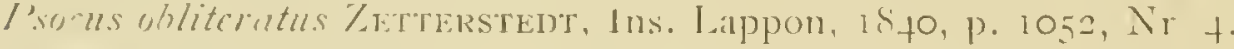

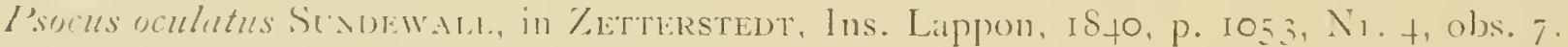

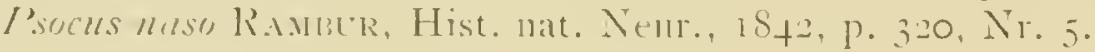

I:lipsocus unipunctutus Mra...). Hacis, Verh. Zool. But. Ges. Wien, Iso6, p. 207.

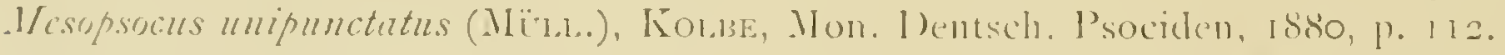

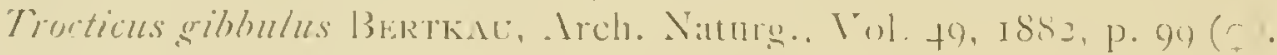

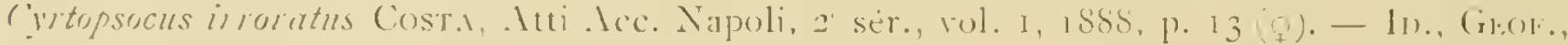

Sard. Mem., IV, asss, p. i6 (2).

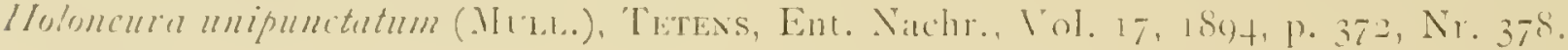

litc., cte.

Coll. Sills: :

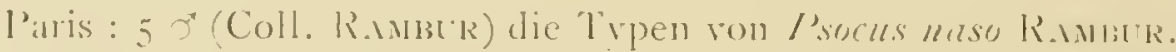

belgien : Spal, 5. Juli. I Coll. SFars; Longchamps bei llatemune, a y Juli; longclamps bei Wrameme, $2 \sigma^{*}$ 1. Alugust.

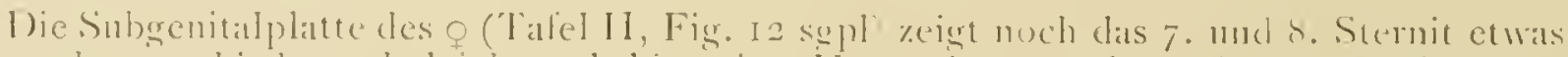

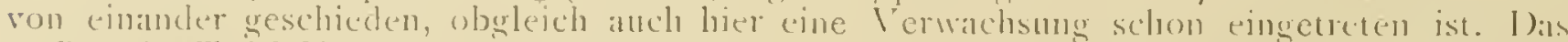
7. Sternit Tafel 1], Fig. I st_) ist eine breite l'latte mit etwas in der Vitte eingedrücktem 
Hinterand, der hier in der Mitte einen breiten sticlartigen Fortsat\% trägt. Hieman schliesst sich dats kleme fast discusirtigne S. Sternit ('lafel 11, fig. 12 st.i.), dass den Stiel ungreift und dessen Rind sich hier dicht dem Stieland anschliesst; die Endhälte ist mit foinen lin\%en

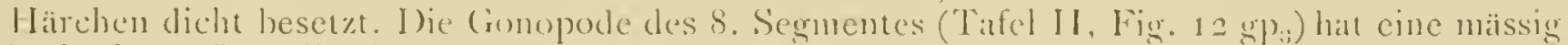

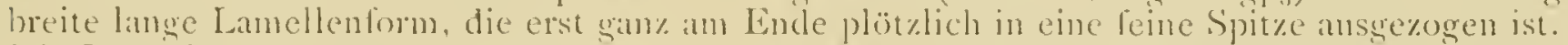

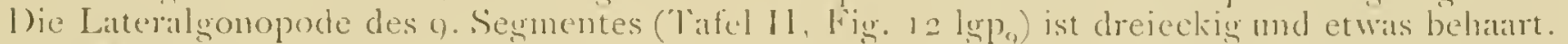
Die Mediangonopode ist eine sehr breite und lange Limblle, die an Ansen- nnd Innenrande nuregelmässig gewölbt, und am Ende gerade abgestuty ist: anssen an binde lindet sich noch

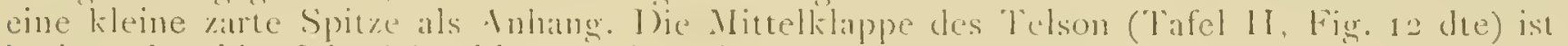
breit rechteckig. I)ic Seitenklippen (lte) sind gross, ("was gewnlstet mud trigen innen an der Basis je ein grosses Simnesfeld mit einer grösseren Anzahl Trichobotrien.

('emis HEMINEURA 'Prens, 18 (8)1.

Hemineura dispar 'Timins, $1 \delta(y) 1$.

(Tafel III, Fig. 13.)

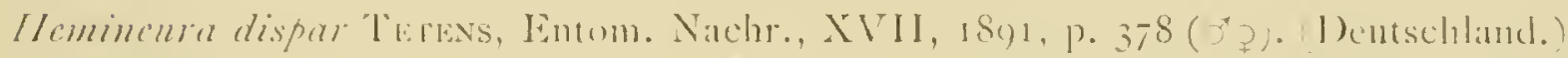

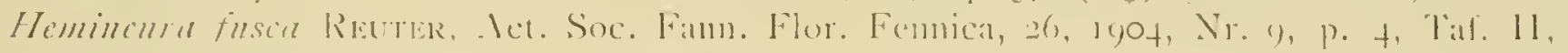
Fig. 2 (ơ) Fimland).

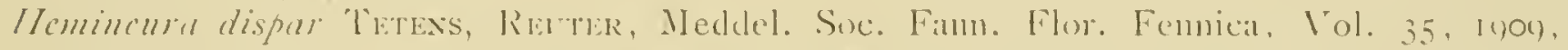

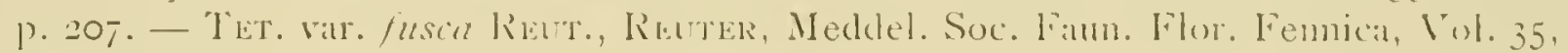
1009, p. 208 .

Coll. Ser.s's :

ond o aus linuland.

Die Subgenitalplatte des $q$ Tafel 11 , Fig. 13 st.., ans dem versehmolzenen 7 . und $s$. Sternit bestehend, ist in der Nihe der Mitte des Hinterrandes in a Spitzen ausere\%gen, \%wischen denen ene tiefe halbkreisfömige Insbuchtung liegt: anf jeder Spit\% inserieren 2 Jorsten: die Behaarung der Subgenitalplate endet ein Stäck vor den Spitzen und der Bucht mit ciner etwas dichteren ( nerreihe ron Halren, die aher an den Seiten nicht mehr als solche ausgeprïgt ist.

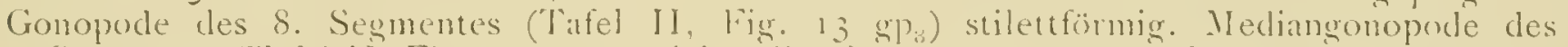

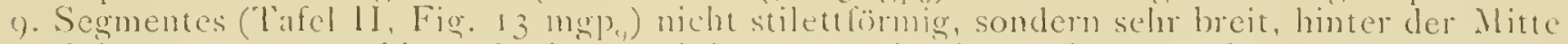
mach innen zn etwas eckig verbreitert und dann etwas in eine breite stmmpfe Spitze ausgezogen. Latteralgonopode des y. Semmentes dreieckig etwas hehatart (Tafel II, Fig. 13 lgph). Die Telsonklappen sind gross, drececlig. die seitlichen tragen an der Basis innen ein kilenes wenig stark entwickeltes Sinnesfeld.

(iemis ELIPSOCUS Haci, 1866.

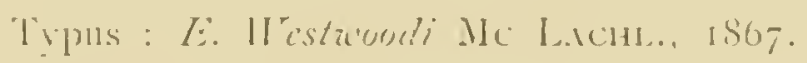

Elipsocus Westwoodi .IC L.icu1.., J 1867.

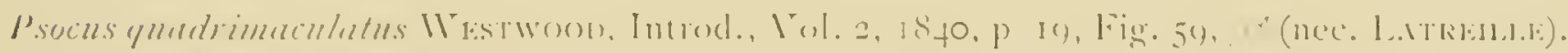

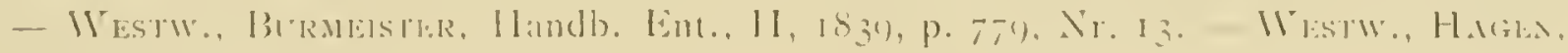
Inn., 1861, p. 24, Ni. 5 . 
Elipsocus quatrimaculatus Westw., H.1gex, Verh. Zool. Bot. Ges. Wien, I866, p. 207.

Elipsocus Il'estroodi Me Laculax, Ent. Mo. Mlag., Vol. 3, IS67, p. 27ł.

Etc., etc.

Coll. Seris's :

Belgien : Halloy bei Ciney, 3. September, I O: Hant Ilarais (Spa), I I September, 3 5\%, 1 q: Geroustère bei Spa. 16. Oktober, I $q$; longchamps bei llatremme, 25. Juli, I $q$ : ferner: 1 o mit der Zentelangabe: "f maculutus."

Elipsocus abietis Kot,ik, I 850.

Elipsucus abietis Kon.me, Mon. 1)entsch. P’soc., ISso, p. II

Coll. Ser.ys :

Wrevbridge, I $\sigma^{*}$.

Belgien: I q, 5. ()ktober; I q, Ende Jimi.

Genus PHILOTARSUS Kol.Jie, i $\$ 80$.

Typus : M\%. Alaviceps Steph., 1836 , Europa.

Philotarsus flaviceps (STEPH., I 836. )

Psucus fluriceps Sterhens, III. Brit. Ent., i 836, p. $124, \mathrm{~N} r .28$.

I'socus striutulus Strephens, Ill. Brit. Ent., 1836 , p. I24, Nr. 27.

Caccilius irroratus Cimets, Brit. Eut., i $836, p .648, \mathrm{Nr} .27$.

I'socus lasiopterus Burmister, Handb. Entom., II, I 839, p. 777, Nr. 5.

I'socus pusillus I) AlMAN, in ZETTERSTEDT, Ins. Lapp., is fo, p. 1053.

I:lipsocus flaviceps (Steph.), Mc Lachlan, Eut. Mo. Mag., V'ol. 3, I867, p. 275.

I'hilutursus flaviceps (Steph.), Kon.13E, Mon. Deutsch. P'sociden, 1880, p. 117, Taf. 2, Fig. 10.

Etc., etc.

Coll. Sriss :

Belgien : Longchanps bei Waremme 7 . und 12 . Angust, $z q, 10$, mit der Angabe "iroratus». - Longchanps bei Waremme. Juli : 2 Exemplare; 25. Juli : + Exemplare; 1. August : 3 Exemplare. 


\section{FAM. PSYLLIPSOCIDAE.}

GemUS PSYLILIPSOCUS SELIS, 1872.

Typus : I's. Kamburi Ser,s, I872, Europal.

Sims Longchames, Ent. Mo. Magaz., Vol. IX, IS72, p. It.5.

Exiffrifin, Zool. Jarb. Sist., 13d 19, 1003, p. 720. - In. Stett. Ent. Zeit., 1906, p. 307. 11., Zool. Anz., Bd 33, 1908, p. 775. - In., Arch. Zool. Expér. V'ol. +1 , lyou (Biospeologica) P. 536. - In., P'alicontographica, Bit 58, 1011, p. 2y2.

\section{Psyllipsocus Ramburi SkLs, 1872.}

Psocus pedicularius (L.), Ranbur, Hist. Nat. Neuropt., 18+2, p. 323 (nec: padiculurius L..

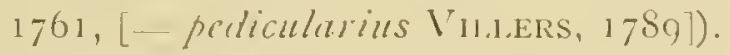

Psyllipsocus Ramburi Ser,s, Ent. Monthly Magal., Vol. IX, is72, p. 1 q6, Fig. B auf p. 145.

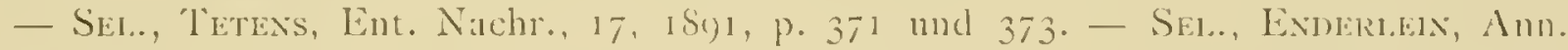

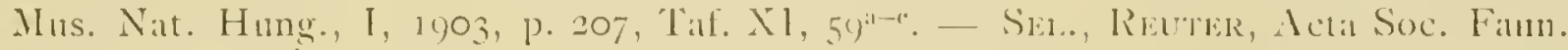

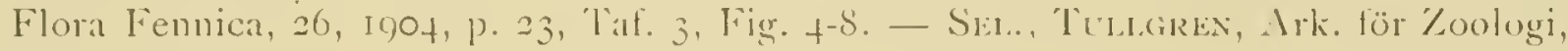
Bd 5 , I909, p. 14 .

Coll. Selsis :

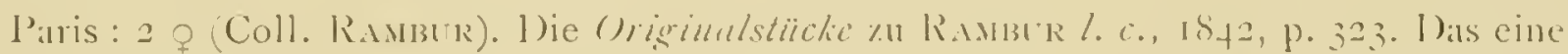
Exemplar trïgt einen Zettel mit der Angabe : I'socus pediculnrius I)e Vinters, dats andere mit der Angabe l'sucus pediculurius.

Diese beiden Exemplare sind \%ugleich dic Typen Sisss.

Aachen, I 4 . September I 888.3 .

I'syllipsucus Ramburi Swas, fehlen die Amaldomen der Seitenklappen des Telson,

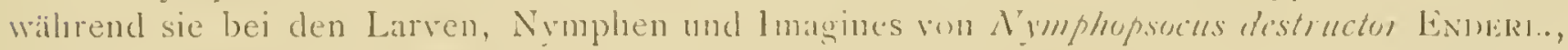

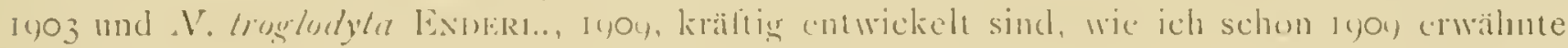
(Arch. Zool. Expér., I'ol. f1, 190y. (Biospeological) p. 536),

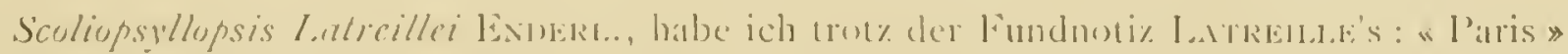
mnter die ansserenrupäischen formen gestellt, da sie meines Erachtens sicher nichn europüisch ist. 
GenIS NYMPHOPSOCUS EximRi, 1903.

Zoolog. An\%, $1903,13 \mathrm{~d}=7, \mathrm{p} .76$.

Nymphopsocus destructor ENInki1., 1903.

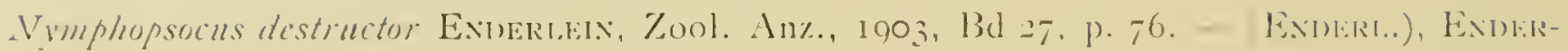

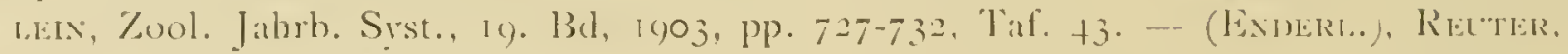

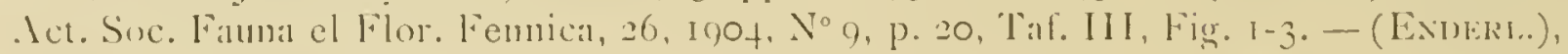

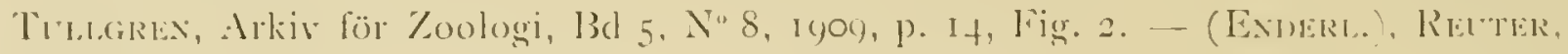
Medd. Soc. Faun. Flor. Fennica, V'ol. 35, 1909, p. 209.

Coll. Ser.'s :

Belgien : Bruxelles. Severan, io. Nov. 1909. In Häusem in grösserer Anzahl.

\section{FAM. TROGIIDAE (ATROPIDAE).}

\section{Genus TROGIUM J I.,., I 798.}

Typus: Tr. pulsutorium (L., I 761 ).

Eximeries, l'alacontographica, 58. Bd, I9 I, p. 353.

Sinonym : Atropos LeAch, I I 5 .

\section{Trogium pulsatorium (L., 1, 76 I).}

Termes pulsatorium Linne, Fama Suecica Ed., II, 1761, p. 474, Nr. 1937.

('othillu studiusa Westwoon, Inn. Mag. Nat. Hist., 18+1, p. fio.

(Cothithe pulsatorie L.), Hacien, Ent. Mo. Mag., Vol. II, IS60, p. 122.

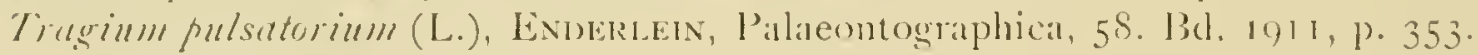

Etc., etc.

Belgien : I o (Coll. Sers's).

\section{Genus HYPERETES KंOıß, I $\$ S O$.}

Hyperetes guestfalicus KOABE, 1880 .

Litteratur etc. vergl. Entikrtein, Res. Swed. Exp. Egypt., 1905, No is, p. 21. Coll. Seirs :

Riigen : Göhren. An Eiche. Einige Exemplare (gesammelt ron (i. ENonktin). 


\title{
FAM. LIPOSCELIDAE (TROCTII)AE).
}

\author{
Genus LIPOSCELIS Norscil., i $\$_{52}$. \\ Typus : L. divinatorius (Müı..., 1776 ).
}

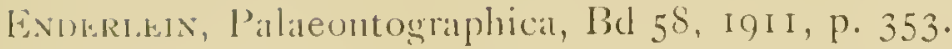

\section{Liposcelis divinatorius (Mü1,1,., 1776$)$.}

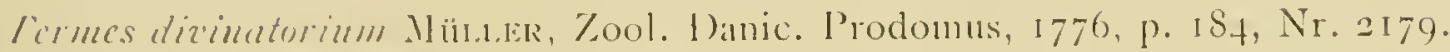

Atropos pulsatorins (Mü1...), Strphens, 111. Brit. Ent., IS 36, p. 128, Nr. 1.

Troctes fatidicus Burnisister, Handb. Ent., Vol. II, I\$39, p. 774 .

liposcelis muscorum Morscmskr, Études Entomol., 1852, pp. in und 20 .

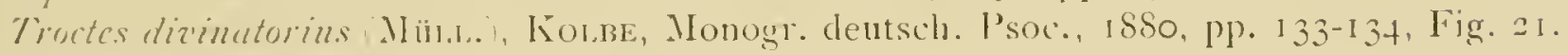

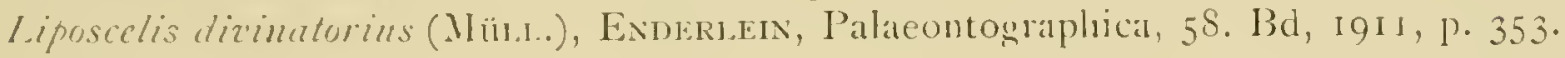

Etc., etc.

Belgien : I q (Coll. SEs.rs).

\section{Liposcelis brunneus Morsch., I 852 .}

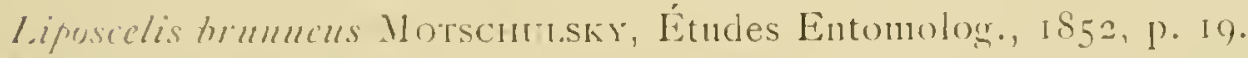

Atropos formicarin II.Mrix, Ent. Mo. Mag., Vol. 11, 1865, p. 121, Nr. 3.

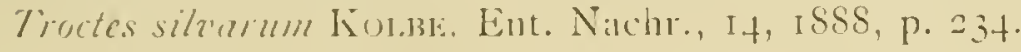

Liposcelis brunucus (10tsch.), Exverlein, Palaeontogr., 58. Bd, 1911, p. 353.

Coll. Selys :

Berlin : Einge Exemplare. Unter Kieferminde. ( ExwlRtax, Herbst ro5. 


\section{Die aussereuropäischen Copeognathen.}

\section{FAM. THYRSOPHORIDAE.}

GEMIS THYRSOPHORUS BIRM., IS, 3 .

Thyrsophorus speciosus Birm., is 38 .

Thysophorns speciosus Burmetster, Handb. d. Entom., II, Bd II, Abt. is $\delta_{3}$, p. 7 \&, Nr. 1.

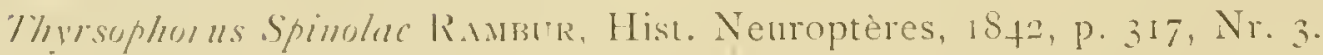

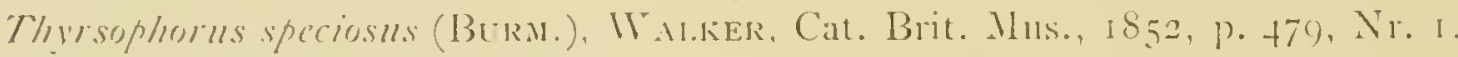

Thresophorus spinolie (R.umb.), Io., ibid., p. tig, Nr.3.

Throsophorus speciosus (Bukn.), Haciex, Srm. Nemopt. North America, 1861, p. 302. (Brkm., In., Stett. Ent. \%eit.. 1\$60, p. 243.

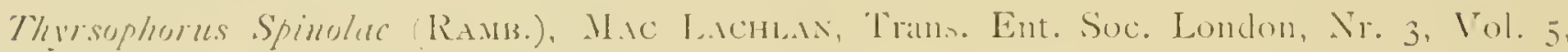
$1860,1) 3+6$.

Ausmabmsweise ist im Vorderliugel $\mathrm{r}_{++}$; mit $m$ wesentlich weiter verschmolzen, so dass $r_{1}$ : erst zwischen m., und $m$, weder aloweigt. Im Hintertügel ist Radialramus mol Iledia allenneist durch eine (Unerater verbunden, vereinzelt kommt eine Verschmelyung beider in einem Punkte und anch eine kur\%e Strecke weit vor (vergl. unten). Beim q hat das 3. Fïblergrlied nu am Ende schrigstehende kraltige Bürstenhatare. Beim ot ist das 3. Fiihlerglied ausser mit diesen P’ubescenzhatren in der ganzen Länge ziemlich dicht mit sehr langen, dünnen und senkrecht abstehenden Haliren beset»t.

Es liegen folgende Stiicke ror:

Coll. Silis's:

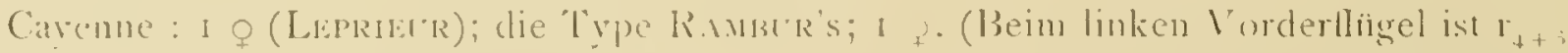

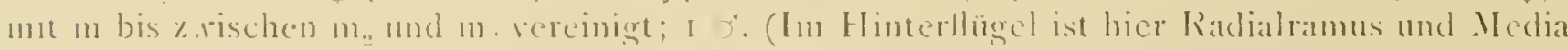
eine kurze Surecke vereinigt.)

Amazonas : Geba, Telle, I 2 , STrumsiner. 
Genus DIC'TYOPSOCUS ENIDEr.., I901.

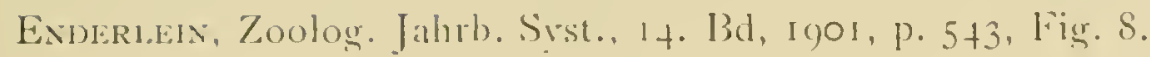

Dictyopsocus pennicornis (BrR., $\left.1 S_{3} S\right)$.

(lalel IV, Fig. IS).

Thysophorus pennicornis Bakmestrer, Handb. d. Entom., II, Bd II, Abt. 1838, p. 782 ,

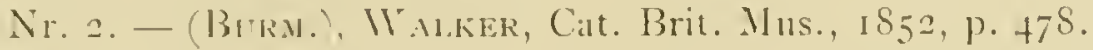

Throphorus rumosus W.M.K1:R, Cat. Brit. Mus., I852, p. $480, \mathrm{Nr} .5$.

Thirsophorns pennicornis (Burm.), Hages, Sym. Nemopt. North America, 1861, p. 302. -

(Burm.), Me Jenchan, Trans. Ent. Soc. London, Nr. 3, Vol. 5, is66, p. 346.

Coll. Sel.'s :

Amazonas: Gebil, Tefle, 3 .

GenIS THYRSOPSOCUS ENHERI, I901.

Thyyrsopsocus aequatorialis ExDERI.., 1901.

Thyrsopsocus aequatorialis ENDERLEIN, Zoolog. Jahrb. Syst., 14. Bd, I9O I, p. 545.

Coll. Seris :

2 o liegen ans dem mittleren Amazonasgebiet ror (Geba, Teffe). 1)ie Species war aus Ecuador beschrieben worden.

\section{FAM. PSOCIDAE.}

Genus CERASTIPSOCUS Kol.13k, $188_{4}$.

Cerustipsocus Koner, Stett. Ent. Zeit., i $88_{4}$, p. 38.

('erastis lion,Be, Stett. Ent. Zcit., $188_{3}$, p.65.

\section{Cerastipsocus venosus (13URм., I 838 ).}

I'sucus renosus Bervieistek, Handl). d. Entom., II. Bd, i838, p. 77 S.

Psocus microphthulmus R.unuer, Hist. Neuroptères, 18.2, p. 321.

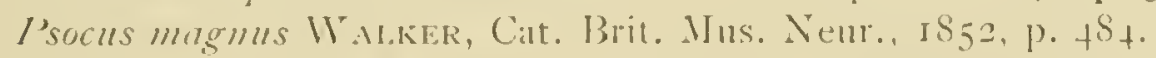

I'sucus zenosus Brrm., H.igis, Sim. Neurop. North America, IS61, p. 10.

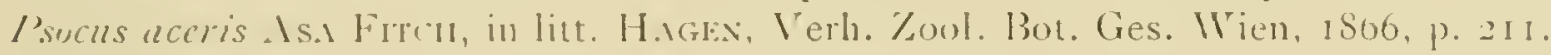

I'socus gregarius H.arris, Ent. Curr., 1869, p. 329. 
('crastis renosa (Burm.), Kol,ze, Stett. Ent. Zeit., 1883, p. 75. - (Burm.) var. mexicana, Konne, Stett. Ent. Zeit., $188_{3}$, p. 75.

Cerrstipsocus renosus (Burn.), Konbe, Stett. Eut. Zeit., IsS4, p. 38. - (Brra.), Id., Arch.

f. Naturgesch., isSS, Bd I, Heft, 2, pp. I53, 178. - Burm.), Banks, Catt. Neur. Ins.

Un. St., 1907, p. 10.

Coll. Selys :

Nord Amerika : 3 q. Originalstücke von /’socus aceris As. Fitch, in litt.; New York, $1 \%$, gesammelt von ĹnLer, i 858 ; Georgien, I q, gesammelt von Morrison.

[Cerastipsocus cubanus nor. spec.].

P. Diese Species steht dem ( $:$ venosus (BuRM.) sehr nahe, ist aber etwas kileiner, der Vorderflügel etwas kiraftiger braun; die hyaline Stelle am proximalen Teil der Areola postica ist etwas grusser; die Fublergeissel ist wesentlich dicker, hesonders das erste (ieisselglied (3. Fühlerglied) die Geisselpubescenz ist kraittiger, dichter und etwas langer, aber ebenfals schräg anliegend (q). Die Schenkel sind etwas mehr

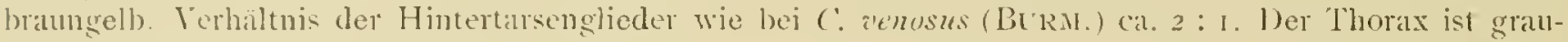
britunlichgelb, das Antedorsum des Mesothorax vou glän\%end schwir\%lich. Das J'erostignna ist weisslichyelb, die proximale Spitye lräunlich.

Vorderllügellinge cat. 6, - $5^{\mathrm{mm}}$. Fühlerlänge cat. 9, $5^{\mathrm{mm}}$. Linnge des 3 . und 4 . Fühlergliedes je $2^{\mathrm{mm}}$.

(Bei ( . venosus (BURn.) ist das dritte $2^{\mathrm{mnn}}$, das . . fast $2^{x}{ }_{4}^{\mathrm{mm}}$ lang).

Cuba. 1 \%

Die Type belindet sich im Naturhistorischen Museum øu Brussel.

\section{Cerastipsocus fuscipennis (Burm., $18_{3} 8$ ).}

I'socus fuscipennis Burneister, Handb. d. Eutom., Bd Il, i $S_{3} 8$, p. 778 . - Burm., Walker, Cat. Brit. Mus. Neur., i $\$ 53$, p. $48+$, Nr. S. - Burm., Haciex, Syrr. Neur. North Am., IS6I, p. 302.

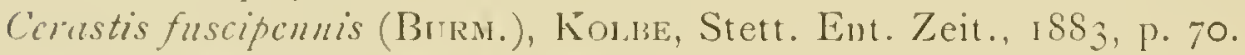

('roustipsucus fuscipennis (Burm.), m.

\section{Coll. Skeys :}

Brasilien : I o gesammelt ron Watther de Sems Longchanps.

Bein) $O^{*}$ ist das ganze erste Geisselglied schwar/branu, wie die übrige Geissel.

\section{Cerastipsocus trifasciatus ('PROv., I $8_{3}$ ).}

I'socus trifasciatus P'rovancher, Pet. Famm. Cam. Nemopt., iS83, p. 65. - P'rov., Id., Nat.

Ciluat. VIII, is $8_{3}$, p. IS6.

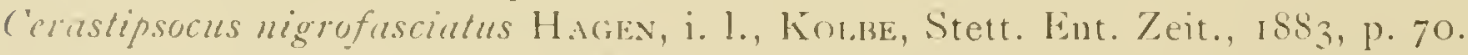

I'sucus speciusus daron, Trans. Imer. Ent. Soc. XI, is 8 , p. to, Pl. IX, Fig. 7.

l'sucus tolteca BAхкs, Jour. New York Ent. Soc., XI, 1903, p. 237 (Mexico) (Var.!).

P'socus trifasciatus l'rov., P'roc. Ent. Soc. Wash., I 904, p. 100 und 202.

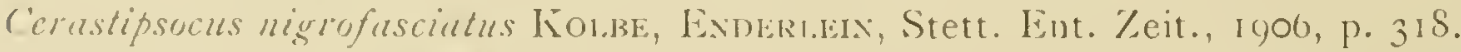

(erastipsocus trifusciatus (Prov.), Banks, Catal. Nemopt. Inst. Un. St., Iyo7, p. 10. 
Die Stücke ans der Coll. Skl,s bestätigen meine Vermutung von 1906, dass Kon lis: den Hagex'schen Namen in litt. ans Sammlnugsstücken kamnte und so veröflentlichte.

Coll. Serris :

Nord Amerika [Amér. Septete]. 2 p, aus der Collection Larkwillk.

Nord Amerika : Georgien, + O', 2 q gesammelt ron Morkisox.

1)iese Stücke sind mit dem Namen P's. Migrofiascintus Hacr. von der Hand H.ncis's versehen.

(ienus LASIOPSOCUS ENIERI., 1907.

Typus: L. Michaclseni Exusre., ryo7. S. IV. Australien.

ENDEkt.en, Ergebn. d. Hamburger. südw.-austr. Forschungreise, Bd I, 1907, p. 234.

Unterscheidet sich ron l'socus Latr. durch dic l'ubescen\% der Adern der Vorderflügel und des Randes in der Aussenhälfte, sowie durcl die Pubescen\% des Randes der Ratlialgabelzelle im Hinterflügel.

Lasiopsocus brevipilosus nо\% spec.

(1:it. III, Fig. 15).

Kopf blass, matt, Clypeus mit ca. I 2 baunen Längslinien, die in der vorderen Hälfte ziemlich gerade längslaufen, in der hintern Hälfe etwas divergieten. Clypeolus blass. I abrum dunkelbram. Scheitel an der feinen Scheitelnaht und am Angentude mit einigen kleinen bramen Flecken. Ocellendreieck stumpfwinklig. klein. Wangen nugefleckt. Angen maissig klein, ïberhalbkugelig and mit einspringendem Winkel alsstehend. Kopfbeharung dichn, lang, fein und ziemlich gerade abstehend. Die beiden Basalglieder der Fühler blass die Geissel sind abgebrochen).

Thorax polient glatt, bramschwaw; das Antedorsum des Mesonotum vor den Parapsidenfurchen lateral fein geblogesäumt. Abdomen dunkelbraun. Coxen blassbraun; Schenkel und Trochanter blass bräunlichgelh; Schienen hell braungelh, Tarsen braun. 1. Hintertarsenglied mit ca. 20, zweites mit 2 ('tendiobothricn. Verhailtnis der Hintertarsenglieder 3: 1. Kilane wenig gehogen, schwar\%, Spitzendrittel gelblich; Zahn an Ende des 2. Drittels, mässig gross, nach der Basis \%u völlig flach verkafend, nach der Spitze zu senkrecht abfallend.

Flügel ziemlich schlank. sc etwats ansserhalb der Vitte des Radialstammes mündend. Verschmelzung von Radialramus und Media mässig kur\%. Radialgabel schmal, von der Vitte

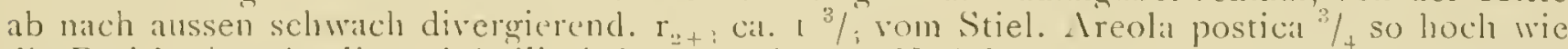
die Basisbreite. Analis und A villaris krenzen siclı an Nodulus gan\% kur\%. Adern fein einreilhig behatart, mit Ansmahme von sc, an und $r_{1}$. Rand an Pterostigna und an der Spitze fein pubesciert. Hinterflügel nur an Rande der \%iemlich breiten Ratlialgabel mit einigen Haaren.

Flügel hy̆alin (Taf. III, Fig. I 5); mit sehr blass bräunlichem Hanch. In Vorderflügel ist Pterostigma mit . Insnahme der proximalen Spitze und Hypostigmalsam dunkelbran; in der Flägelmitte sind die Adem breit bram gesänm, basalwäls selır breit gesämmt. Adern bram. Membran etwas rotirisierend and in gewisser Belenchtung schwach weisslich opalisierend.

Förperlänge (trocken) $3^{\text {nsm! }}$.

Vorderfliigellänge $5^{\text {mim }}$.

Coll. SElys :

Capland (?). I $q$. 
Genus PSOCUS Latr., 1794.

Psocus taprobanes HAG., 1858 .

I'socus taprobanes Hagria, Verh. Zool. Bot. Ges. W'ien, 1858, p. 773 (Ceylon - H.ir., ID.,

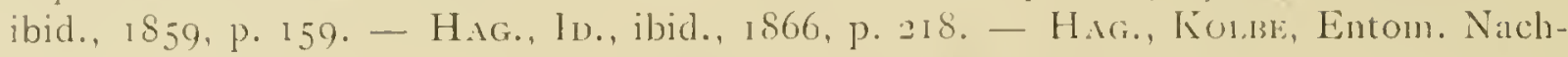

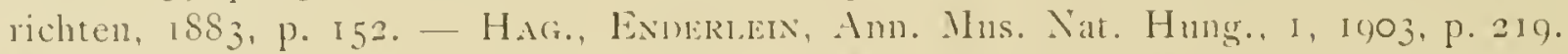
Taf. f, Fig. 9 und 10). (Cevlon, Hinter Indien, - Hak., In., Notes from the Leyden Mus., 29, 1907, p. I13. (Amman, Java). - HAG., Lb., ibid., 34, 1912. 1. 161. (Annam, Java).

Die Beschreibung dieser Species mit ihren Tarietäten habe ich 1903 gregeben und wiederhole sie hier nicht, da die Typen völlig danit ïbereinstimmen.

Ceylon: 3 o, 1 q. Nietner. Die 'lypen Hagkes.

Psocus obtusus HaG., 1858 .

('laf. III, Fig. I6).

I'socus ohtusus HAGEN, Verh. d. Zool. Bot. Ges. Wien, is 58 , p. 474. - Hag., Iı., ibid., IS59, P. 202. - HAG., ID., ibid., IS66, p. 216.

o. Kopf mit den Fühlern und Palpen braungelb. Fühler lang, mit langer abstehender Pubescenz, Spitzenhälfte gebräunt. Scheitelnaht kräftig.

Thorax gelbbraun, oben dunkelbram mit braungelben Suturen. Abdomen dunkelbraun, Spitze braungelb, Beine braungelb.

Flügel ('âf. 111, Fig. 16) hyalin mit mehr oder weniger bräunlichgranem Ton. Adern braun mit den gewöhnlichen gelben Stellen. P'terostigma im Enddrittel braun. Hypostigmalsaum schmal und braun. Scheitel der Pterostigma's flach grewölbt. I'ereinigung von Radial. ramus und Media in einer knrzen Strecke. Je ein ganz unscheinbarer blassbrauner Flecken am Ende des Nodulus, ein (Onerfleck durch die Mitte der Endhälfte ron $11+c u$; ebenso ein schmaler Sam an den beiden Strecken der Media an der 1)iscoidalzelle. Areola postica sehr breit; der Scheitel ungewöhnlich breit: der absteigende 'Tei] von cu, fast senkrecht. Radialgabel ziemlich schmal, parallel, an Encle schwach divergierend; Länge der Gabel ca. doppelt so lang als der Stiel. Membran rot bis grün irisierend..

Körperlänge (trocken) ca. $2^{\mathrm{mm}}$.

Vorderflïgellänge $t^{\text {mm }}$.

Coll. SEl,ys :

Ceylon : Rambodde, 2 o gesammelı ron Nierner. Die 'lypen Hagen's.

[Psocus cubitalis nov. spece.].

P'sucus obtusus H.ig., Exmlkiem, Inn. Mus. Nat. Hung., I, 1903, p. 227, Taf. 1Y', Fig. 14 (q) (nec: P's. obtusus Hi(i.).

Hinter Indien. Singapore. I897. 5 .

Iypen im Bubapester Musem, i Exempla davon in meinen Besit\%.

Burch Vergleich dieser Art mit den Typen won Psocus oblusus Haci. stellte sich die Verschiedenheit herans. Die beschreibung und Abbildumg findet sich an der citierten stelle. Auffillig ist bei dieser Species 
der ungewohnlich lange 2. Cubitalast $\left(\mathrm{eu}_{2}\right)$, der schriug nach auswen und hinten gerichtet ist und den

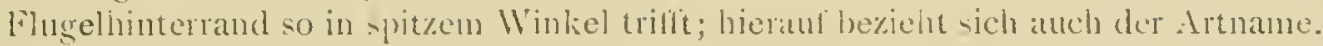

Fehlt in Coll. SELs's.

Psocus Hageni Baxks, I904.

(laif. III, Fig. I 7 ).

l'socus Hagchi Banks, l'roc. Ent. Soc. Wish., VI, Igot, p. 202. (Oestl. Ver. Staten).

I'socus contaminatus Hagks, S:-n. Nem. N. Amer., 1861, p. 10 (nec Stephexs, I830). (New York, Maryland, Washington und Mexico (Vera Cruz.)

In der Collection Sis,s findet sich cine der HAGw'schen 'Typen und zwar das Exemplar von Vera Cruz.

đ. Kopf bell bräunlichgelb, Clypeus mit sehr feinen bräunlichen längslinien. ()cellenbasis braun. Labrum ockergelblich, in der Mitte braun. Augen für das männliche Geschlecht relativ klein, über habkugelig abstehend. Maxillapalpus bram, die beiden Endglieder schwar\%. Fühler schwärzlich, die P'ubescen\% ling, mässig dicht und senkrecht abstehend; die beiden Basalglieder gelbbraun.

Thorax schwarzbraun, Scutellarleisten bräunlichgelb, Schienen rötlichbraun, Tarsen braun, Schenkel bräunlichgelb, Endspit\%e schwärzlich. Verhältnis der Hintertarsenglieder $3: 1$. Abdomen gelbbram .

Vorderfügel (Taf. III, Fig. 17) hyalin; dunkelbram ist : das Pterostigma mit Ausmahme des Basalviertels, der Endteil der Zelle Cu, und von hier aus ein ziemlich breiter Randsam bis zn $\mathrm{r}_{4+}$, der in der Ungebung ron $\mathrm{cn}_{2}$, und am Rande in den \%ellen $M_{1}$ und $\mathrm{I}_{2}$ den Rand nicht berüht, sondern hyalin unterbrochen ist; 4 . mud 6 . Sechstel der Analzelle und die Spitz. der Axillarzelle. Scheitel des Pterostigma etwa in der Mitte, stumpfwinklig und abgerundet. Radialramus und Media in einen l'unkte verschmolzen oder durch eine sehr kurze Querader verbunden. Mediangabel schmal, fist parallel, $r_{z+}$; fast doppelt so lang wie der Stiel. Areola postica hoch. Scheitel schmal mit der Media rerschmolzen. cu. kur\%. Hinterthögel hyalin. IIembran stark speckig glänzend, und etwas rot bis grün irisicrend.

Körperlänge (trocken) $3,5^{\mathrm{mm} n}$.

Vorderflügellinge 6.0 mm.

Coll. Sel,ys :

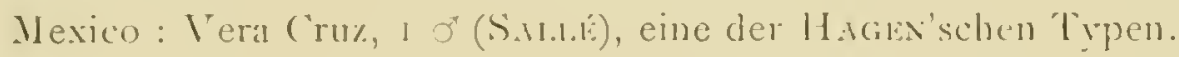

Psocus novaescotiae WAK., I852.

('Tal. III, Fig. If; Taf. IV, Fig. I9).

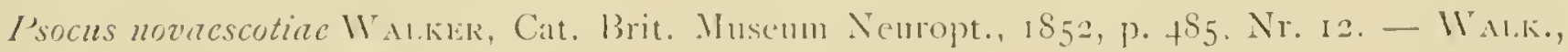
HAGEx, Syn. Neur. N. A., IS61, p. I1.

Psocus cratacgi Asa Fitcu (Manuscr.), Hacikx, Verh. Zool. Bot. Ges. Wien, is66, 1). 213.

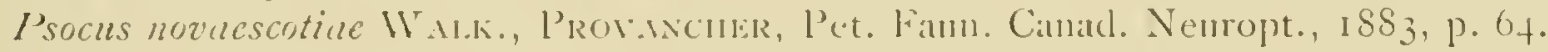

Kopf (Taf. IV', Fig. Iq) grelblich, ein Fleck in der Mitte jeder Scheitelhälfte und das Labrum ohne die Seitendrittel schwar\%. Endglieder des l’alpus braun. Augen mässig gross, überhalbkugelig abstehend, schwar\%. Fïhler schwar\%, die z ersten Glieder gelblich; länger als der Vorderflügel, P'ubescen\% kur\% und dicht. 'Thorax gelblich, die Dorsa und Antedorsa des Meso- mond Metanotmm poliert schwam. Abdomen ockergelblich mit schwamen ()uerbinden und 
Zeichnungen. Beine hell gelblich, Spitzen der Schienen und die Tarsen schwarz. Verhälnis der Hintertarsenglieder $2 \%$ : I.

Flïgel hralin Taf. III, Fig. 1 +). Subcosta mündet dentlich in r. Pterostigma schwärzlich, Basis und äusserste Spitze weisslich. Adem bram, an einigen Stellen weisslich. Hypostignalsamm bram und an Scheitel des l'terostigma ein bramner Fleck, der mit dem Pterostigma zusammenhängt und fist bis an die Gabelnng des Radialramus reicht. Ein blass branner Fleck durch die Mitte der \%, lle Cu und An, sovie ein kleiner hellbraner Fleck am Nodulus. Scheitel der Areola postical schmal; basalwärts davon ein breiter blasser brämlicher

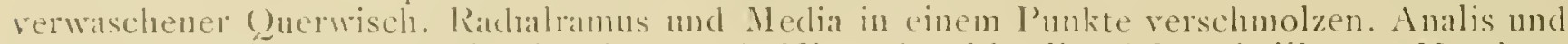
Axillaris am Ende ein wenig sich krentend. Hinterlligel halin, Adern hellbram. Membran lebhatt in allen Farben irisierend.

Körperlänge trocken) $3,5^{\mathrm{rnm}}$.

Vorderllïgellänge $5 \%$ min.

Coll. Sruss :

Nord Amerikil: 1 (Coll. Latren,le); 1 q. Mit der Noti\% : I's. cratuegi A. Fitch, remutich die Stücke, die HAgEN zu seiner Notiz veranlasste.

\section{GenIS TRICHADENOTECNUM ENILRı., I909.}

'lipus: Tr. scxpunctutum (L.). Europa.

Exulktis, Boll. Lab. Zoul. agr. l'ortici, Vol. III, rgog, p. 329.

Trichadenotecnum circulare (HAC., I 554$)$.

(Taf. I, Fig. 26).

I'socus circularis Hagex, Verh. \%ool. Bot. Ges. Wien, i859, p. zor (q). (Ceylon). - Hafr., ID., iloid., 1866, p. $212(q)$.

I'socus quinquepunctutus MAC LACH1.Ax, Ent. Monthl. Mag., Vol. 9, 1872, p. $75\left(0^{7}\right)$.

l'socus circularis H.ag., Enderlian, Ann. Mus. Nat. Hung., Bd r, 1903, p. 224, 'Taf. XIII, Fig. $69(0)$.

I'socus qumqucpunctutus Mc Lachı., Exbrkikin, Ann. Nus. Nat. Hung., I, iq03, p. 225. Taf. $13, \mathrm{Fig} .67\left(0^{\circ}\right)$.

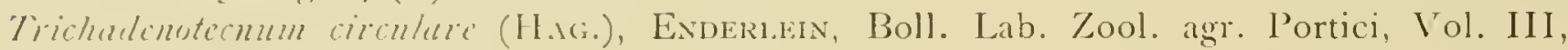
I 909 , p. 330.

Die vorliegende Type HAGEN's ist noch etwas dunkler und kräftiger in der Flügelfärbung sowie etwas kleiner als die Stucke die mir rgoz vorlagen und die ich abbildete. Die Type ist sehr schlecht erhalten und ich habe ans den Bruchstücken ein Praeparat angefertigt, nach dem jeh die Figur ausfïhrte.

Flïgrellängre $3 \%$ mm.

Coll. Sritys :

Ceylon : 1 g gesammelt ron Nierner; die Type Hagen's.

Auch jetzt halte ich noch den Tr. quinqucpunctatum (IC L.AcHi.., IS72) für clas of von Tr. circulare H.1(r.), wie ich schon Ig03 loc. cit. vermutet habe. Das loc. cit. Fig. 69 abgebildete o stellt die Vermittlung zwischen der 'Type und dem ot dar. 
Genus TAENIOStigma ENDEri., Igol.

Typus: T. clongertum, Hacr., 1858 . Ceylon.

Taeniostigma elongatum ( $\mathrm{HAG}_{\mathrm{A}}, \mathrm{I}_{5} S$ ).

Psocus clongutus Hagen, Verh. Zool. Bot. Ges. Wien, IS58, p. t7t. - Hacr., Mr., ibid., I 859 , p. $200\left(0^{*}, q\right)$. - HAG., lo., ibit, 1806, p. 2I3.

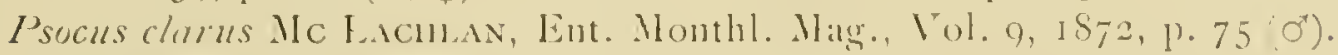

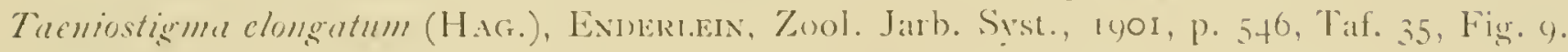
Tacuiostigmu clerum (Hagr.), In., ibid.

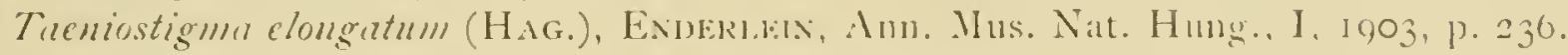

Coll. Sel.ys :

Ceylon : 1 o, 1 q, Nietrer. Typen Haciex's.

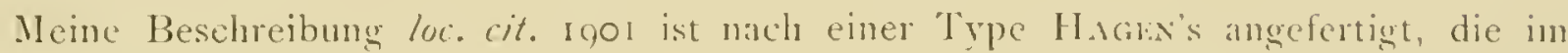
Berliner Zoologischen Musemm sich findet.

Genus HEMIPSOCUS SLi,y Longch,, 1872.

Selys Loxgchanps, Eut. Mo. Mag., Vol. 9, is $8_{72-1} S_{73}$, p. I 45 , Fig. 4 A.

Kolde, Stett. Ent. Zeit., $188_{3}$, p. 79.

Enderi.tin, Ann. Mus. Nat. Hung., Vol. 1, ig03, p. 233.

\section{Hemipsocus chloroticus ( $\left.\mathrm{HAc}_{\mathrm{r}}, \mathrm{I}_{5} \delta_{5} 8\right)$.}

(Taf. IV, Fig. 2I).

Psocus chloroticus Hagen, Verh. Zool. Bot. Ges. Wien, i 858, p. $+7+$ (Ceylon). - Hagr. In., ibicl, I 859 , p. 200. - HAG., ID., ibicl., 1866, p. 2i2. - HAG., MAC LACHan, Ent. Mo. Mag., Vol. 9, I 872, 1). 77 . Note 3 .

Homipsocus chloroticus (HAG.), Slass Lovicumas, Ent. Mo. Mig., Vol. 9, IS 72, p. I45. p. + i.

Hcmipsocus hyalinus Enneriein, Stett. Ent. Zeit., igo6, p. 3II. (Japan). - Exinkr.., Okanoto, 'Trans. Sapporo Nat. Hist. Sue., Vol. II, 1907, p. I 35.

Coll. Selys :

Eine der 'lypen Hages's liegt in der Collection SEars vor, und es reigt sich, dass es nicht die Form mit den Iderpnnkten ist, wie ich ly03 annahm, sonclem die hraline Species, die ich 1906 ron Japan beschrieb. //. chloroticus ist daher bisher ans ceylon und Japan bekannt. Die Flägelabbildung ist nach cinem Exemplatr ans Japan angelertigt.

Ceylon : Nietrek, 1 q, die Type Hager's.

Für die Form mit pmbtierten Adern ist daher ein nener Name einzufïhren; ich widme sie dem Andenken des Antors der Gattumg. 
[Hemipsocus selysianus nor. spec.].

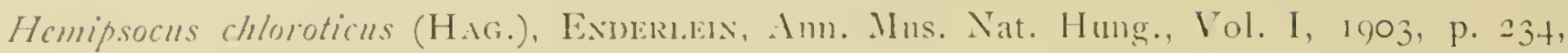
Taf. +. Fig. I $7^{\text {lt-c; }}$ Taf. 6, Fig. $1^{-d-f}$.

Homipsocus Sclysinums m.

Hinter Indien. Singapere.

Mlit der Varietit.

Var. luridus ExbirL., 1903.

Hemipsocus chloroticus (Hag.) var. luritus Enideridin, loc. cit. p. 235, Taf. 4, Fig. 17a.

Deutsen Neu Guinea. Lemion in Berlinhafen, und Simbang am Ihun-Golf.

Fehlt in Coll. SE⿺r's.

Genus GRAPHOPSOCUS Kiolize, ISS2.

Graphopsocus uniformis (HAG., I859)

I'socus uniformis HAgren, Verh. Zool. Bot. Ges. Wien, is 59, p. 200.

Stcurpsocus uniformis HAgex, Verh. Zool. Bot. Ges. Wien, 1866 , pp. zis und 2 Ig.

Grophapsocus uniformis (Hag.), Endera.en, Am. Mus. Nat. Hung., I, I903, p. 242, Taf. V,

Fig. $20($ (o).

Coll. Siriss :

Ceylon : I ơ 2 q gesammelt ron Nietner. Die 'Typen Hagen's.

Meine Beschreibung ron 1903 wurde nach einer Type HAges's im Berliner Zoologischen Museum angefertigt.

\section{FAM. C A E C I L I I D A E.}

\section{Genus POLYPSOCUS Hag., i 866.}

Polypsocus Hagen, Verh. Zool. Bot. Ges. Wien, 1866, p. 203.

I'tilopsocus ENIJER EIN, Zool. Jahrb. Sist., XIV', I900, p. 153.

Polypsocus corruptus (HAcr., I 861 ).

('Taf. IV, Fig. 22 und 23).

Psocus commptus Hagke, Nemopt. North America, r861, p. 13 (07).

l'sucus ubruptus Hacise, Nenropt. North Americal, 1861 , p. I3 (f).

Polypsocus comptus (Hag.), Hacex, Verh. Zoul. Bot. Ges. Wien, 1866, p. zi . - (Hag.), Banks, Cat. Nemr. Ins. United St., 1907, p. S.

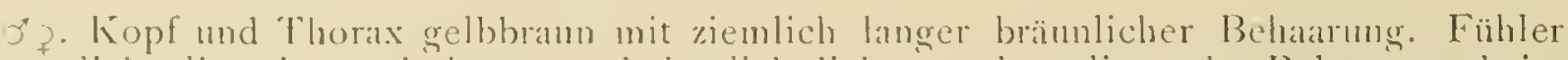
blass bräunlichgelb, mit mässig langer und ziemlich dichter meln anliegender Behaarmug. beim 
$o^{7}$ sind die Crlieder in der Spitzenhälfte gebräunt und die Behaurmug etwas länger. Augen beim o" (Fig. 2I) sehr gross, kugelig abstehend, beim q klein, hablibelig abstehend (Fig. 23). Beine hell rosgelb, Hinterschenkel etwals gebriunt.

Flügel braun ('Taf. IV, Fig. 22), beim der Spit\%e mit einer mässig breiten hyalinen Querbinde, hein o ist dieselbe seh schmal und nur in der vorderen Hälfe ansgebildet (var. abruptus Hag, 'at. IV, Fig. 23). Adern dunkelbram, zweireihig behart, Analis einreihig behart. Stigma mit Ausnahme der Spitze dunkelbraun, mit kn\%er dichter Behaarmg; ebenso ein schmaler Randsaum bis in die Ireola postica mit kurzer dichter Behaarung. Randbehaarung riemlich ling. Länge des Mediamgabelstieles stark variirend, meist sehr lang und die Gabel sehr ku\%. Länge des sehr kuren Stieles der Radialgabel etwas variirend. Grösse stark variirend.

Körperlänge (trocken) durchschnittlich $2^{\text {mmm }}$.

Vorderflügellänge durchschnittlich $3 \% \frac{\mathrm{mm}}{\mathrm{m}}$.

Coll. Sillys :

Nord America : Wrashington, i $\%$, I 858 , gesammelt von Osten-Sackex (Type von I'socus abruptus HAG.); Georgien, $+\mathrm{O}^{*}$ und I $\mathrm{o}$ gesammelt von Morkison.

Eine anffällige Geäderaberration zcigt folgendes Stück :

Stiel der Radialgabel fehlend, beide Radialgabeläste in einem Punkte (links) oder durch kurze Querader (rechts) verbunden.

Amér. septentrionale: i q. Coll. Latreilic.

Dieses Stück gelangte aus der Sammlung von Latreilite in die von Fonscorombe, ron dieser in die Sammlung ron Ramburk und schliesslich in die Sammlung ron Sel,ys Longchamps.

Genus HAGENiElla Enderi., 1903.

Tỵpus: H. zonita (HAG., I859), Ceylon.

Hageniella Enderuent, Anm. Mus. Nat. Hung., I, 1903, p. 25s. - ID., Palaeontographical, I9II, P. 3 II.

Die Diagnose für diese Gattung ist loc. cit. 1903 unrichtig nnd ich stellte sie loc. cit. 1911 richtig.

Diese Gattung unterscheidet sich von lipipsocus H.1G., 1866 [Typus IE. ciliatus (Picr., HAG., I 856)] durch die ungezähnten Klauen. Zu Epipsocus gehört LE ciliatus Pict., Hag., I 856 (fossil), E. nepos Evideri., igoo, Peru und E. murcus. Endert., igo3, Hinterindien, zu Hageniella gehört: H. zonuta (H.AG., 1859, Ceylon, HI. funesta (ENink1.., 1903), Australien, H. marginata (ExDerl., I903), Neu Guinea, E. villosa (ENdikı, I903), Australien, H. molesta (HAG., I S59), Ceylon und H. lanatu (HAG., I 859), Ceylon.

Der Radialramus und die Media sind wie bei Epipsocus durch eine mehr oder weniger kurze Querader verbunden.

Epips, delicatus (HAG., I859), Cerlon und Ep. ruserrs (HAG., I859), Cerlon gehören durch die Verschmelzung von Radialramus und Media zn Pscudocaccilius ExDler1.., igo3. 


\section{Hageniella zonata (H..., I 859$)$.}

(Taf. V', Fig. 24), Fig. 8 .

Psocus zomutus H.hen, Verl. Zool. Bot. Ges. Wien, i 859. p. 204 (Ceylon).

Epipsocus zonutus (H.ici.), HAGEN, Verh. Zool. Bot. Ges. Wicn, 1866, pp. 219 und 207. (HAg.), Id., Stett Ent. Zeit., 1882, p. 2 So.

Epipsocus fuscofusciatus Exnert.en, Ann. Mus. Nat. Hung., I, 1903, p. $25+$ (Ceylon).

Hageniella zomata (HAg.), Exinerieix, Ann. Mus. Nat. Hung., I, igo3, p. 258. - (HAg.),

Id., Palaeontographica, I 9 II, p. 31 I.

Zwei Trpen Hagex's liegen in der Skus'schen Sammlung vor. Ich ersehe hieraus, dass der ron mir ans Cerlon beschriebene Epips. fuscufusciatus Evilk1.. I 903 mit dieser Species identisch ist. Die Sexualdifferenzen sind die gewöhnlichen : die Augen des or sind auffällig gross und stark abstebend, und die Fühlerbeharung des ơ sehr lang; beim o sind die Augen mässig klein, die Fühlerbehamung ebenfalts lang, aber kïrer als beim ot und weniger dicht.

l) ie braune Flïgelbinde (Taf. $V$, Fig. 24), die noch ausserhalb des Endes des ersten Flügeldrittels der Vorderflugel durchquert (nicht wie HAGEx schreibt : «fascia transversa ad basin fumosa "), ist beim ơ sebr blass. Angen beim s゙ schwar, beim o braun.

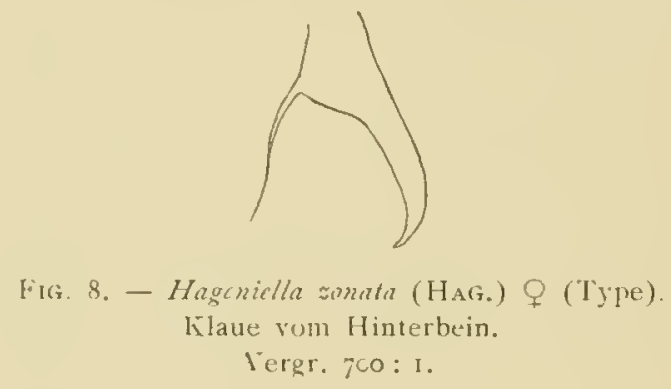

Ich verweise im übrigen auf meine Beschreibung loc. cit. 1913. 1). 25t und auf die Abbildung des weiblichen Vorderllïgels nach einer der Typen.

Körperlänge (trocken) o $1,5^{\mathrm{mm}}$, Q I, $7^{\mathrm{mm}}$.

Vorderfliggellänge $O^{x} 3,2^{1 \mathrm{~mm}}$, O 2.9 $9^{\mathrm{mm}}$ (nach loc. cit. 1903, p. 25t-300).

Coll. Selys :

Ceylon : Rambodde, I Ơ, I q gesammelt ron Nietner. Die Typen Hagen's.

Hageniella molesta (H.is, 1859$)$.

(Taf. V, Fig. 25).

Psocus molestus Hagex, Verh. Zool. Bot. Ges. Wrien, i 859, p. 203.

Epipsucus molestus (H.ig.), Hacien, Verh. Zool. Bot. Ges. Wien, is66, pp. 2 is und 208. (Hac.), Id., Stett. Ent. Zeit., 1888, p. 280.

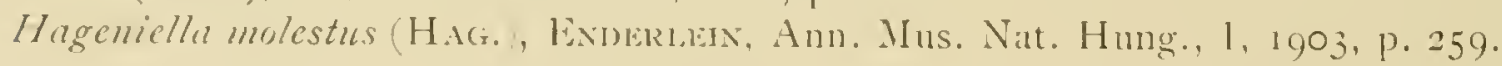

Q. Her ganze Körper blass brangelb. Augen uässig gross, etwas abstehend, bram bis dunkelbraum. Scheitelnaht kriftig cingedrickt. Kopf und Thorax mit sehr blasser, langer Behatrmg. Fïhler mit ziemlich langer und nicht sehr dichter Behamung. Beine sehr blass. 
Flüget halin (Taf. V, Fig. 25), mit etwas gelblich tribem Tone. Aderu blass bräunlich gelb, mit zwei Reihen sehr langer Hare, nur die Tualis mbehant. P'terostigma lang und flach,

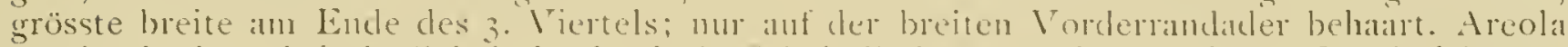
postica breit und thach, Scheitel seh. Hach, Scheitelhöhe ctwas länger als der Scheitelabstand ron der Media. ()nerader zwischen Kaddalranus mul Media durch eine ziemlich langer (Onerader verbunden. Radialgabelstiel fatst doppelt so lang wie $r_{.+.}$. Radlialgabel stark divergierend. $\mathrm{Cu}_{2}$ kurz. Auf dem Aussenrand kreuzen sich die Harre. Aussenrandinler sehr breit. Hinterflügel hyälin, Adern sehr blass: Rand behaint, . Kern der Spite mit eingen Härchem.

Ǩ̈rperlängge (trocken) I, t"'m.

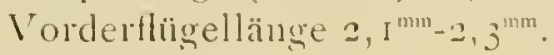

Coll. Seliss :

Ceylon : Rambodde, 2 q gesammelt ron Niwisk. Die 'Typen Hagis's.

Genus CAECILIUS Curt., $1 S_{37}$.

Caecilius aridus (H..., $\left.{ }_{1} \delta_{5} \delta\right)$.

Psocus aridus Hagex, Verh. Zool. Bot. Ges. Wien, 1858, p. 474. - In. ibil., is 59 , p. 202. ('uccilius uridus (HAg.), Hagen, Verh. Zool. Bot. Cies. Wien, 1866, p. 205. -. (HAg.),

Exderlein, Ann. Mus. Nat. Hung., I, 1903, p. 267, Taf. I3, Fig. 68.

Coll. SEl.Y's :

Ceylon : a q gesammelt von NiETrek. I)ie Typen HAGEx's, die mit meiner Abbildung und Beschreibung ron 1903 übereinstimmen.

Genus LACHESILLA IVEST., $18+0$.

Typus: L. perlicularia (L., I 761 ).

Pterodela KouzE, isSo.

Graphocaccilius ExDERi.., I 900.

Leptopsocus Riut., I S99 vergl. in dieser Abh., p. I6.

Die Gattungsmuterschiede, die Kon.rbi loc. cit. angibt, sind nicht stichhaltig, besonders findet man die Vereinigung von Radialranus und Media im Vorderflügel in einem Punkte nur sehr selten, meist sind beide Adern eine längere Strecke mit einander vereinigt.

Wie ich schon frïher hinwies, ist las wichtigste Characteristicum neben dem Fehlen der Flügelpubescenz (sie ist nur bej einigen Arten sehr spirlich entwickelt) der IBesitz eines Zahnes an den Klatuen, das sie allen übrigen Gattungen des Caecilidentribus (accilimi

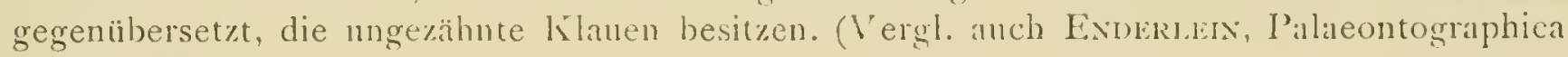
I 9 II, p. 289.)

\section{Lachesilla pedicularia $(1, ., 1761)$.}

Hemcrobius pedicularius Lixib, Famna Suec, 2, 1761, p. $3 S_{+}$(Emopa).

Psocus salicis HAcis; Nemopt. North America, 1861, p. 13 (Mss. Fiтch) (Nord America).

Psocus geologus Wrish, l'roc. Acad. Nat. Sc. Philat., 1862, p. 362 (Nord America). -

ID., I'roc. Ent. Soc. P'hilid., 1863 , p. 168 (Nord America).

Psocus pusillus Hark, Ent. Corr., I864, p. 33 I (Nord America). 
Pterodela pedicularia (L.), Kon.Be, Jahresber., Zool. Sect. Westf. (IS97-So), iSSO, p. 1 I 8. Ciecilius pediculirius (L.), HAGEx, l'strche, III, ISSI, p. 195 (Nord America).

Pterodela pedicularis (L.), Basks, Cat. Neur. Inst. Un. St., ryo7, p. 7.

Lachesilla pedicularia (L.), ExDerusis, in dieser Abhandlung, p. I6.

Coll. Seris's :

Nordamerika : I $q$.

Wahrschemlich eine der 'Typen HAGer's des Psocus saliciis HAG, in der Sammlung Skiss. Auf einem grinen Zettel findet sich die Noti\%: "P. salicis A. Fitcu».

Genus DYPSOCUS HAg., is66.

Typus: D. colcoptratus (HaG., I 858 ), Ceylon.

Dypsocus coleoptratus ( $\left.\mathrm{HAG}_{\mathrm{A}}, \mathrm{I}_{5} 8\right)$.

Psocus coleoptratus HAGEx, Verls. Zool. Bot. Ges. W'ien, I858, p. 474. - I D., ibid., 1859 , p. 205. Dypsocus coleoptritus (HAG.), HaGex, Verh. Zool. Bot. Ges. Wien, I 866, pp. 2 I 2 und 207. (HAG.), Exderlein, Ann. Mus. Nat. Hung., I, 1903, p. 250, Taf. V', Fig. 24.

Coll. Selis :

Ceylon : i ơ, I q gesammelt von Nietner. Typen Hages's.

Genus CALOPSOCUS HAGen, is66.

Typus : C. infelix (HAG., I $8_{5} 8$ ), Ceylon, Indien, Bismarck Archipel.

Calopsocus infelix ( $\mathrm{HAGEN}_{\mathrm{AGE}} \mathrm{I}_{5} \mathrm{~S}_{\text {.) }}$

1'socus infelix HAGen, Verh. Zool. Bot. Ges. Wien, 1858, p. 475. - Id., ibid., I 859, pp. 204, 205 .

Calopsocus infelix (Hag.), Hagen, Verl. Zool. Bot. Ges. Wien, r866, pp. 2 I 4 und 206. (Hag.), Enderlein, Ann. Mus. Nat. Hung. I, igo3, p. 246, Taf. VI, p. $22^{\mathrm{a}-\mathrm{c}}\left(0^{\top} \phi\right)$. (HAg.), Id., Zool. Jahrb. Srst., 20. Bd, I9 I4, p. I06, Taf. 7, Fig. 1, ơ. Bismarck Archipel (Insel Rillum).

Coll. Selys :

Ceylon : I đ̛, I $q$ gesammelt von Nietner. Typen Hagen's.

Auch im Berliner Zool. Nus. findet sich ein Pärchen 'Trpen.

Genus ECTOPSOCUS Mac Lachl., I $\$ 99$.

Ectopsocus piger ( $\mathrm{HAG}_{\text {. }}$, 1859).

Ysucus piger HaGex, Verh. Zool. Bot. Ges. Wien, I 859, p. 202.

Peripsocus piger (Hag.), Hagen, Verb. Zool. Bot. Ges. Wien, I866, pp. 216 und 210 . -

(HaG.), Exderlein, Ann. Mus. Nat. Hung., I, I903, p. 292.

Ectopsocus piger (HAG.), m.

Hell rostbräunlich, Kopf und Thorax oben etwas gedunkelt. Fïhler und Beine blasser. Augen gross, halbkugelig abstehend, etwas dunkler. Ocellen klein, leuchtend rötlich, zu gleichseitigem Dreieck angeordnet. Clypens ziemlich stark gewölbt. Beine blass branngelb. 
Flügel graulualin, Aclem gelbbram. Stigmasack braun. Adern unbehaırt. Radialramus und Media im Vorderflügel durch eine selnr kurze (Querater, in Hinterfligel durch eine lange Querader verbunden. Pterostigna gleichbreit, $f$ mal so lang wie breit, $r_{\text {, parallel }}$.ur Costa. Radialgabel lang und schmal, wenig divergierend, $r_{1}$ etwas lïnger als der Gabelstiel. Membran stark grün bis rötlich irisierend.

Vorderflïgellänge $2,4^{\text {man }}$.

Coll. Seirs :

Ceylon : Rambodde, a q gesammelt von Nierstr. Die 'Typen Hagex's.

\section{FAM. M Y O P S O C I A E.}

Gemus LICHENOMIMA ENDERL, 1910.

Lichenomima lugens (HAci, IS61).

(Taf. IV, Fig. 20.)

Psocus lugens Hagen, Syn. Neur. North America, i86i, p. 9.

Myopsocus lugens (HAG.), HaGes, Verh. Zool. Bot. Ges. Wien, r6, i866, p. 2 го.

Psocus mubilus Harris, Ent. Corr., is69, p. 332.

Myopsocus lugens (HAG.), Banks, Cat. Neur. Ins. United St., I907, p. 8.

Lichenomima lugens (HAG.), Enderlein, Sitzungsber. Ges. Naturf. Fr., I9I0, p. 66.

q. Kopf matt dunkelbraun, Clypens stark gewölbt dunkelbraun mit hell gelbbramen Längslinien. Am Hinterhaupt einige hellere Stellen. Augen nicht gross, halbkugelig abstelıend, schwar\% und unbehart. Clypeolus und Labrum hell gelbbraun. Labrum mit dumkelbrauner Mitte. Fühler dümn, braun, kur\% behaart.

Thorax bram, die Leisten hell gelbbraun. Beine braun, Schenkel am Ende des 2. Drittels mit einem schmalen hell gelbbramem Ring. Schienen etwas heller, an der Spitze dunkelbraun. 1. Tarsenglied blass braungelb, 2 und 3 . Glied braum. Abdomen braun bis rostbraun.

Vorderflügel (Iaf. V, Fig. 25) schwärzlich dunkelbrann, Rand und Adern durch zahlreiche kleine weisse Flecken unterbrochen. Der übrige Flügel mit Ausnalme eines mässig breiten Aussenrandsammes mit einer ziemlich hyalinen Masermng. Pterostigma dreieckig, ebenfalls mit weisslich hyaliner Maserung. Areola postica am Scheitel kurz gestielt. Radialramus und Media durch eine kur\%e Querader verbunden. Hinterflïgel rauchig, Radialramus und Media durch eine mässig lange (Juerader verbunden. Membran des Vorderflïgels matt, der Hinterflïgel, stark blaugrïn bis gellogrïn irisierend.

Körperlänge (trocken) $2 .{ }^{3} /{ }_{+}^{\mathrm{mm}}$.

Vorderflügellänge $3,8^{\mathrm{mm}}$.

Coll. Seits:

Nord Amerikı: Washington, I $q$.

Die Type Hagen's. Nach der Angabe Hagev's wude dies Stück I 57 ron Ostex Sackin gesammelt. 


\section{Lichenomima sparsa ( $\mathrm{H}_{\mathrm{i}} \mathrm{r} ., \mathrm{i} 86 \mathrm{I}$ ).}

('Tafel $T$, Fig. 27, 28), Fig. 9.

Psocus spursus H.tgex, Sym. Neur. North America, I86I, p. 8. - (HAG.), Id., Verh. Zool. Bot. Ges. Wien, 16, 1866, p. 218.

Psocus infuscatus Hakris, Ent. Corr., 1869, p. 332.

Myopsocus spursus (HAG.), Provaxcher, Pet. Faun. Canad. Nemropt., is83, p. 63. - (HaG.), B.xks, Cat. Neur. Ins. U.S., I907, p. S.

Lichenomimu sparsa (H.ı..), Exnerl., Sitzungsber. Ges. Naturf. Freunde, Jhrg. 1910, p. 66.

Kopf matt hell braungelb, Clypeus mit feinen bramen Längslinien. Scheitel in der Mitte mit einem braunen Längslleck, an den Seiten mit je 2 Längsreihen kleiner brauner P’ïnktchen. Scheitehnaht deutlich. Scheitel beim o kïrer. Kopf bein ơ ganz matt schwar\%. Angen mässig gross, halbkugelig abstehend, beim ơ sehr gross und halbkugelig abstehend. Fühler lang, blass braungelb, häufig mit dunklerer Zeichnung; beim o mit kurzer dichter Pubescenz, beim ơ mit etwas längerer und ein wenig mehr abstehender Pubescen\%.

Thorax braungelb, Antedorsum und 1)orsum des Mesonotum mit Ausnahme der Suturen matt schwar\%. Schenkel dunkelbram, am Ende des 2. Drittels mit einem hell braungelben

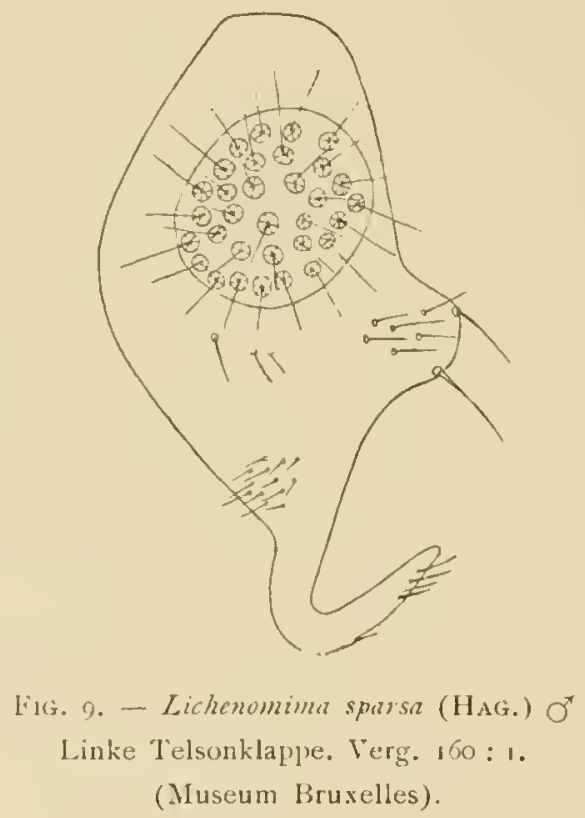

Ringe. Scisienen braungelb, Spitze schwarz. 1. Tarsenglied hell braungelb, 2. schwarz. Subgenitalplatte des o* (Taf. $\mathrm{r}$, Fig. 28) nach binten verjüngt und mit einem scheibenförmigen hinten eingebuchteten Anhang. Die beiden Penis-Sclerite (Taf. V, Fig. 27) lang, am Ende etwas zugespitz und innen ror dem Ende mit einem halbkreisförmigen Anhang. Seitliche Telsonklappen (Fig. 9) an Ende mit einem wach innen gebogenen Haken der an Ende einige Börstchen trägt, und mit grossem, runden Simuesfeld.

Vorderflügel hyalin grau, mit dichter dunkelbrauner Bespritzung, die sich in einem breiten Randsam der Aussenrandzellen zu branschwar\%en Flecken verdichtet, die inneu mit schmalen hyatinem Band gresäumt sind. Adern alternierend schwar\% und gelblich gefleckt. I’terostigma gelbbraun bis gelblichbraun, nur die Spitze und die begrenzenden Adern gefleckt. 
Hinterfligel etwas grau getribt, Adern bräunlich, V'orderrandader alternierend dunkelbrann und grelblich getleckt. Membran des Vorderflïgels matt, des Hinterfliigels intensir irisierend.

Körperlänge ca. $23 / t^{m m}$.

Vorderflïgellänge $+3 / 4-5^{\mathrm{mm}}\left(\mathrm{O}^{\mathrm{m}} 4^{\mathrm{mm}}\right)$.

Coll. Sel.'s :

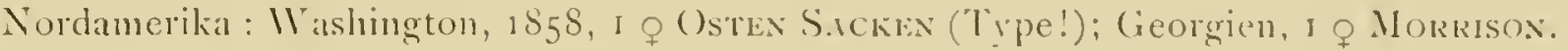

Zum Vergleich hatte ich noch einige Stücle, die ich Hern N. Basks verdanke.

\section{FAM. LEPIDOPSOCIDAE.}

\section{SUBF.M. PERIENTOMINAE.}

Genus PERIENTOMUM HAG., i 865.

'Yyus : P. trichopteryn (HAG., 1859), Ceylon.

Perientonum trichopteryx (H.AG., 1859).

Fig. Io.

Amphientonum trichoptery. Hagen, Verh. Zool. Bot. Ges. Wien, I859, p. 205 (Ceylon).

Pericntonum trichoptery (HAG.), HAGex, Ent. Monthl. Mag., II, I865. pp. I 5 I und 152.

Amphientonum trichopteryx (HAG.), HAGEN, Verh. Zool. Bot. Ges. Wien, IS66, p. 204. -

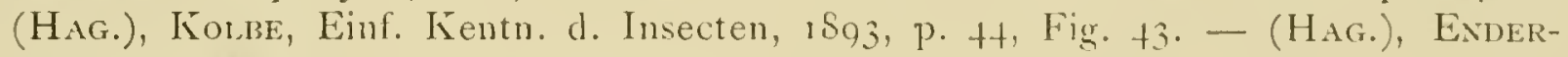
Lein, Amn. Mus. Nat. Hung., Bd I, I 903, pp. 3 I I und 325.

Perientonum trichopteryx (HAg.), Enderi.ein, Spolia Zeylanica, Vol. IV, i go6, p. 90.

Q. Kopf hell graubraungelb. Clypeus lilein, kurz und wonig gewölbt. Ocellen rötlich, weit getremut. Palpen hell braugelb, Endglied schwarzbran. Schlälen felılen. Augen gross, etwas abstehend.

Thorax gelbbraun. Beine hell rostgelblich, Hinterbeine etwas dunkler und mehr bräunlich. Abdomen bram.

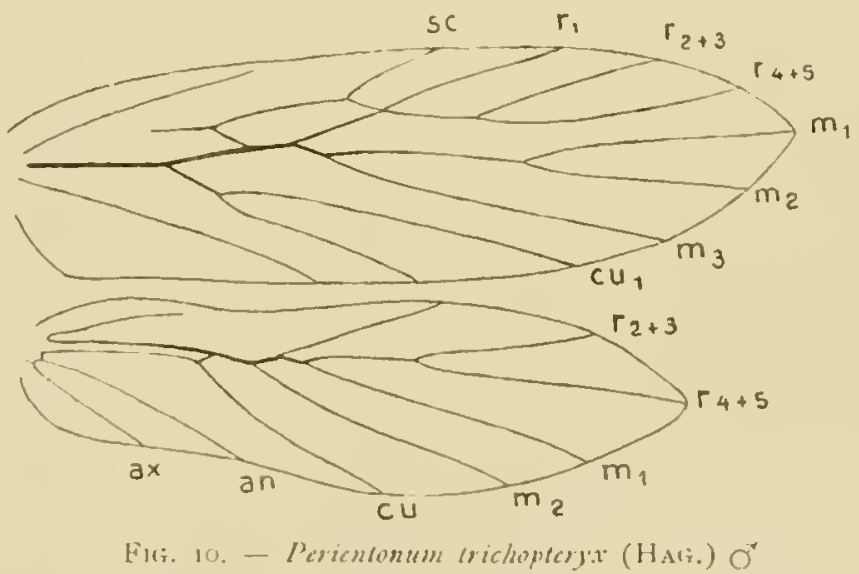

Vorderllïgel-Membran blass brämlich hralin. I)ie Schuppen sind grösstenteils abgerieben, nur an der Spitze finden sich einige Schuppen von gambräunlicher Farbe, die am 
Rande in der Radialgabelyelle eine silberne Auflellung erkennen lassen, die an Perientonum cevlonicum ExDERı. Igo6 ans Cerlon erinnert. Hinterflïgel hralin. In Vorderflügel (Fig. 10) $r_{r}$ in einem l'unkte mit dem Radialgabelstiel verschmolzen. $m$, endet in die Flügelspitze. Im Hinterfligel mündet $r_{4+5}$ in die Flügelspitye. $r_{2+3}$ ist etwa $1 / 4$ rom Stiel.

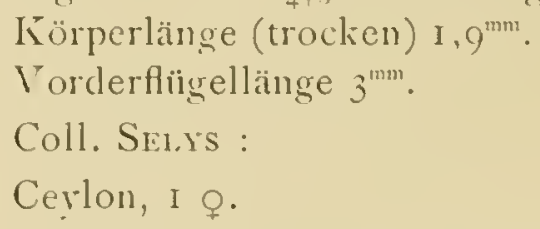

Eine der HAgEx'schen Trpen, nach HAgen in Rambodde von NiErNer gesammelt. Das Exemplar ist in sehr schlechtem Zustande. Ha HAGEN nichts näheres über die Zeichnungsverteilung auf dem Vorderflügel sagt, ist diese Art vorläufig noch ungenügend festgelegt.

\title{
FAM. P S Y L L I P S O C I D A E.
}

\section{Genus SCOLIOPSYLLOPSIS Enderl., 1912.}

\author{
Endertein, 7oolog. Anz., 39. Bd, 1912, p. 304. \\ Typus : S. Latrcillei Enderl., I9I2.
}

Drei Ocellen. Kopf sehr lang gestreckt, besonders die Stirn und das vorn halbkreisförmig gerundete Labrum. Clypeus ében, Torderrand gerade, breit und etwas anfgerichtet. Augen unbehaart. Fühler etwis länger als der. Vorderflügel, die 3 ersten Geisselglieder sehr lang, die ïbrigen sehr kurz; melır als 13 gliedrig (die Spitze abgebrochen).

Prothorax kirz, aber fast so breit wie der Nesothorax, sehr hoch und ron oben gut sichtbar. Tarsen 3 gliedrig, Klanen ungezähnt.

Sc am Ende gebogen und mündet ein Stück vor dem Pterostigma in r. Das distale Stück lang. Radialramus und Ifedia eine Strecke mit einander verschmolzen. Zwischen Pterostigma an der Gabelungstelle und Radialgabelstiel eine Querader. Media 3 ästig; $\mathrm{Cl}_{2}$ ziemlich lang. Axillaris einfach. Analis und Axillaris enden in einem Punkte. Im Hinterfingel ist Radialramus und Media gegabelt; $\mathrm{ax}_{\mathrm{I}}$ ist sehr lang und mündet nicht weit von an; ein kur\%er Gabelast vor der Mitte ist wohl $\mathrm{ax}_{2}$.

\section{Scoliopsyllopsis Latreillei ENDERL., I9 I 2.}

(Taf. IV, Fig. 21; Taf. V, Fig. 30).

Scoliopsyllopsis Latrcillci Enderientr, Zoolog. Anz., 39. Bd, 1912, p. 304.

Kopf ziemlich glatt. Labrun etwas länger als breit, vorn halbkreisförmig gerundet. Clypeolus mässig kurz. Clypeus nicht gewölbt, gan\% eben, Vorderrand aufgeworfen. Seitenränder nach hinten mässig stark convergierend. Hinterrand ziemlich breit gerade abgestutzt und in der Mitte mässig stirk eingedrückt. Ocellen zu einem ziemliclı grossem fast rechtwinkligen (wenig spitzer) Dreieck angeordnet. Abstand der hinteren Ocellen etwa halb so lang wie ihr Abstand vom Augenrand. 
Augen halbkugelig abstehend, hinten rom Hinterhauptsand tangiert, unpubesciert. Scheitelnaht sehr fein, etwas erhaben liegend, da die beiden Scheitelhälften leicht eingedrückt sind. Hinterhanpt llach schneidenartig zusammengedriuckt, last grerade, und die Kante abgerundet. Clypens, Librum und Scheitel nur mit selur liurzer spärlicher l’ubescenz. Fühler selur dïnn, 3. Füllerglied fast halb so lang wie der Vorderllügel, +. Glied fast $\%$; des 3. , das 5. ca. $2 /$; des 4 , die ibrigen sehr kul, ungefihr hinter dem I 5. Glied abgebrochen; länge der vorhandenen Glieder zusammen etwas länger als clic Vorderflïgellänge.

Pronotum etwas breiter als der Scheitel zwischen den Augen, sehr stark von vorn nach hinten zusammengedrückt muct die Ebene des Scheitels und Mesonotums ein ganzes Stiick nach oben \%u überragend. Der ganze Thorax mupubesciert und ziemlich glatt. Abdomen mit sehr spärlicher und sehr kurzer, an der Spitze mit sehr langer l'ubescen\%. 1. Hintertarsenglied mit cál. 23 ganz flachen, undeutlichen und undeutich gezithnten Ctenidiobothrien, das 2. mit 4, das 3. und 2 solchen; die \%u jedem Ctenichiobothrium gehörige Borste ist kräftig und ca. $3 /$ des Tarsendurchmessers. Klaue schlank, klein, fast gerade, Spitze schwach gebogen; ungerähut. Tarsus sonst sehr spärlich behaurt. Schienen nur imen mit sehr kurzen und spärlichen Hairen, ohne Ctenidiobothrien; imnen mit + Endspornen. Verhältnis der Hintertarsenglieder ca. $6: 1: 3 /$

Fliigel (Tafel IV, Fig. 21; Tafel V, Fig. 30) sehr glatt. Ranchder mit microscopisch feinen, schlanken, anliegenden, fist schuppenartig aussehenden Gebilden dicht beset». Adern sehr dünn. sc an der Basis etwas wellig gebogen. Die Querader zwischen Pterostigma und Radialgabelstiel triflt die Mitte des letateren. Der ganze Radialgabelsticl ist nicht gan\% so lang wie $\mathrm{r}_{2+3}$. cu, gleichmässig gewölbt. Zwischen ax und an abnorm bei einem Flügel eine Querader an Ende des ersten $Y$ iertels.

Kopf hell ockergelblich; dunkelbram ist eine schmale Linie mitten durch die grossen Wangen zwischen Angenvorderrand und den Clypens-Seiten nalie am Vorderrande, die etwas länger als der längste Augendurchmesser ist; ebenso ein Randsanm an der hinteren Hälfte des Clypensseitentandes; die Ocellen, die Fühler mit Ausnahme der 2 ersten und der Balsalhälfte des 3. Gliedes. Thorax hell ockergelb, Dorsum und Antedorsm des Mesonotum ist bräunlich. Abdomen hell ockergelblich, Oberseite mit Ausnahme der Spitze dicht schwarz punktiert. Beine blass ockergelblich, Tarsen schwarzbram. Klanen brämlich. Flügel wasserklar, die Spitzen von $r_{4+5}, r_{2+3}$ und $r_{1}$ blassbraun gesäumt, der P'terostigmalteil von sc gänzlicl, bräunlich gesäumt. Adern und Randader blassbräunlich. Membran stark grïn bis rot irisierend und ausserdem mit intensivem farblosen Glanz.

Körperlänge (trocken) $t^{\mathrm{mm}}$.

Vorderflügellänge $5,7^{\text {mm }}$.

Coll. Selys :

Frankreich, Paris, 1 gesammelt von Latreilı.E.

Das ausserordentlich interessante Exemplar fand sich in der SEI.'s'schen Sammlung mit der Angabe "Coll. Latrent1.1* " und einem 2. Zettel mit der Notiz " Paris " von der Hand Latrcille's unter Psucus longicornis F. neben den 'Typen von Psocus lincutus I.ATR. (E Ps. longicornis F.), mit welcher Art sie auch in Grösse und Erscheinung eine gewisse Aehnlichkeit hat. Vermutlich ist das Tier ron LATREILle falsch etikettiert oder es ist durch Zufall eingeschleppt worden; eine Vermutung äber die wirkliche Heimat des Tieres lässt sich nicht aussprechen. 


\title{
FAM PSOQUILLIDAE.
}

\author{
Genus PSOQUILLA Hag., I 865.
}

Psoquilla HAgex, Ent. Monthly Mag., Vol. II, i 865 , p. I 23.

P'terostigma fehlt. $r_{1}$ ausgelizdet. Radialramus durch eine kurze Querader mit m verbunden, der Basalabschnitt ron $\mathrm{rr}$ aber selnr undeutlich und daher die Zelle R undeutlich geschlossen. Meclia 3 ästig, $m_{3}$ proximal der Radiomediancuerader. Cubitun gegabclt, an und ax getrennt endend. Adern und Rand mit relativ liriftigen Haaren. Nihe dem Hinterrande auch einge Hare in der Membran. Hinterflügel stark reduciert, kum, ohne Adern und ohne Behaarung.

Die Gattung Psocatropos Ribiga 1899 ist übrigens neben Psoquilla zu halten, sie unterscheidet sich trot\% aller Aclervariabilitat doch constant durch dien ungegabeiten Culitus; ihr gehören Ps. Lachlani Rrb. I\$g9 (Italien), Ps. slossonae (BANks 1900) [Nord Amerilia] und Ps. micrups (ENderL. 1903) [West Afrika, Formosa] an.

\section{[Psoquilla marginepunctata $\left.\mathrm{HAG}_{\text {., I }} 865\right]$.}

Psoquilla marginepunctata Hagen, Ent. Monthly Mag., Vol. II, I865, p. I23. - HAG., Mc Lachi.an, Ent. Monthly Mag., Vol. II, i866, p. 197, Pl. II, Fig. +. - Hag., Kolbe in Rostock, Neur. germ., i 888, p. 189.

(Fehlt in Coll. Selys).

Diese interessante Form, die in England auf Kornböden, auf denen indisches Korn aufgestapelt war, grefunden wurde, galt immer als ein aussereuropäisches eingeschlepptes Inselit. Durch die Tatsache, dass mir aus Paraguay (San Bernardino) einige Stücke vorliegen, hat sich diese Vermutung bestätigt.

\section{ERRATA \& ADDENDA}

Seite 26, unten Mesopsocus unipunctatus (Müll. I764): (Tafel II, lïg. 12.) stellen.

Seite $30, \%$ andern : \%eile 8 ron unten «Tragium» in : «Trugium».

Seite 39, H. chloroticus ('Tafel V, Fig. «29») statt : «2I 》.

Tafel II, liig. $8:$ « Bertkania »statt : «Berkania». 


\section{SYSTEMATISCHE LISTE}

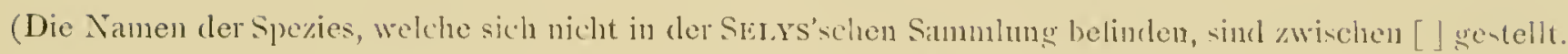

Ein Stern be\%cichet, diass der Typun in (lerselben Sammlung ist.)

Seite.

I. Europäische Copeognathen.

Subordo : ISOTECNOMERA 5

Fam.: PSOCIDAE 5

Subfam. : PSOCINAE

Genus Psocus Latr., I 794

gibbosus (Sul\%., I776)

nebulosus Steph., i 836

bipunctatus ( $\mathrm{L}$., I76I)

"quadrimaculatus Latr., I794

Genus Neopsocus Kalbe, I SS2

rhenanus Kolbe, i $\$ S 2$

Genus Amphigerontia Kolbe, is8o

"bifasciata (Latr., I799)

fasciata ( $\mathrm{F} ., 1787$ )

* variegata (Latr., 1799)

Grichadenotecnum Euderl., 1900

sexpunctatum (L., I 76 I) II

majus (Kolbe, Loens) 1 )

Genus Clematostigma Enderl., 1906 II

*morio (Latr., 1794)

Subfam. : STENOLSOCINAE

Genus Stenopsocus Hag., I866 12

immaculatus (Steph., 1836) I2

Lachlani Kolbe 12

stigmaticus (Iuh. et Labr., I $84^{6}$ ) I3

Genus Graphopsocus Kolbe, 1880 I3

cruciatus (L., 1768 ) 13

Fam.: CAECILIIDAE 14

Subfan, : C.ALCIIIINAE

Genus Caecilius Curt., 1837 . 14

flavidus (Stepli., 1836) If

obsoletus (Steph., 1836) If

- fuscopterus (Latr., 1799) I5

piceus Kolbe, ${ } \delta_{32}$

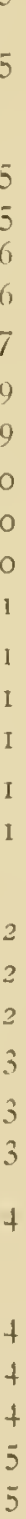
(jentis Trichopsocus K()]be ISSS 15 Dalii (Mac Lachlan, I 867 ) 15 Ge'luc Lacliesilla Westw., $18+0 \quad 15$ perlicularia $(1, ., 1761)$ I6 var. brevipenuis Enderl., I903 19 var. fatidical (1.., 1758$) \quad 19$ quercus Kolbe, 1SS2) 19

Sulufan. : PERII'SOCINAli

Genus Peripsocus Hag., $1866 \quad 20$ phaeopterus (Steph., IS 36$)$ subfasciatus (Ran11b., $1 \mathcal{S}_{+2}$ ) 20 allogguttatus (Dalus., I823) 2 I

Subfun. : BERTK\UIINAE

Genus Bertkauia Kolbc, ISS2 2 I lucifuga (Ramb., I $8+2$ ) 2I

Subfan. : REUTERLLLINAE

Genus Reuterella Euclerl., I903 23 helvinacula (Ender]., IgoI) 24

Subordo :

\section{HETEROTECNOMERA 25 \\ CRYPTODERATA \\ I)ERMOSTIGMATOPHORA}

\section{Fam. : MESOPSOCIDAE 25 \\ Subfan. : LEPTODEILINAE 25 \\ (renus Leptodella Rent., I994 25} fusciceps (Reut., I893) 25

Subfam. : MLSOPSOCINAE 26

Genus Mesopsocus liolbe, $1880 \quad 26$ unipunctatus (Müll., 1-6 4 ) 26

Genus Ilemineura leteus, I891 27 dispar Tetens, I891 27

Genus Elipsocus Hag., I $\$ 66 \quad 27$ Westwoodi Mac Lachl., I867 27 abietis Kolbe, ISSO 


\begin{tabular}{|c|c|}
\hline $\begin{array}{l}\text { Genus Philotarsus Kolbe, I8So } \\
\text { flaviceps (Steph., I } 8_{3} 6 \text {; }\end{array}$ & \\
\hline Subordo : IDELODERATA & \\
\hline Fam. : PSYLLIPSOCIDAE & \\
\hline $\begin{array}{l}\text { Genus Psyllipsocus Selys, } 1572 \\
\text { Ramburi Selys, } 1872\end{array}$ & \\
\hline $\begin{array}{l}\text { Genus Nymphopsocus Enderl., 1903 } \\
\text { destructor Eirlerl., } 1903\end{array}$ & \\
\hline Fam. : TROGIIDAE & \\
\hline $\begin{array}{l}\text { Genus Trogium I1l., } 1798 \\
\text { pulsatorimm }(\mathrm{L} ., \mathrm{1} 76 \mathrm{r})\end{array}$ & \\
\hline $\begin{array}{l}\text { Genus Hyperetes Kolbe, I } 888 \\
\text { guestfalicus Kolbe, I } 880\end{array}$ & \\
\hline Fam. : LIPOSCELIDAE & \\
\hline $\begin{array}{l}\text { Genus Liposcelis Motsch., I } 852 \\
\text { divinatorius (Müll., I } 776 \text { ) } \\
\text { brumneus Motsch., } 1852\end{array}$ & \\
\hline
\end{tabular}

\section{Aussereuropäische Copeognathen.}

Fam. : THYRSOPHORIDAE 32

Genus Thyrsophorus Burm., $1 \delta_{3} \delta \quad 3^{2}$ speciosus Burm., $183^{8} \quad 3^{2}$

Genus Dictyopsocus Enderl,, I901 33 pennicornis (Burm., 1838 ) 33

Genus Thyrsopsocus Enderl., 190133 aequatorialis Enderl., 190I 33

\section{Fam. : PSOCIDAE 33}

Genus Cerastipsocus Kolbe, $1884 \quad 33$ venosus (Burm, I 838 ) 33 [cubanus nov. spec.] 34 fuccipennis (Burm., 1838) 34 trifasciatus (Prov , $188_{3}$ ) 34

Genus Lasiopsocus Enderl., $1907 \quad 35$

•brevipilosus nov. spec. $\quad 35$

Genus Psocus Latr., 1794 $\quad 36$

"taprobanes Hag., $185^{8} \quad 3^{6}$

"obtusus Hag., $1858 \quad 3^{6}$

[cubitalis nov, spec.] $\quad 3^{6}$

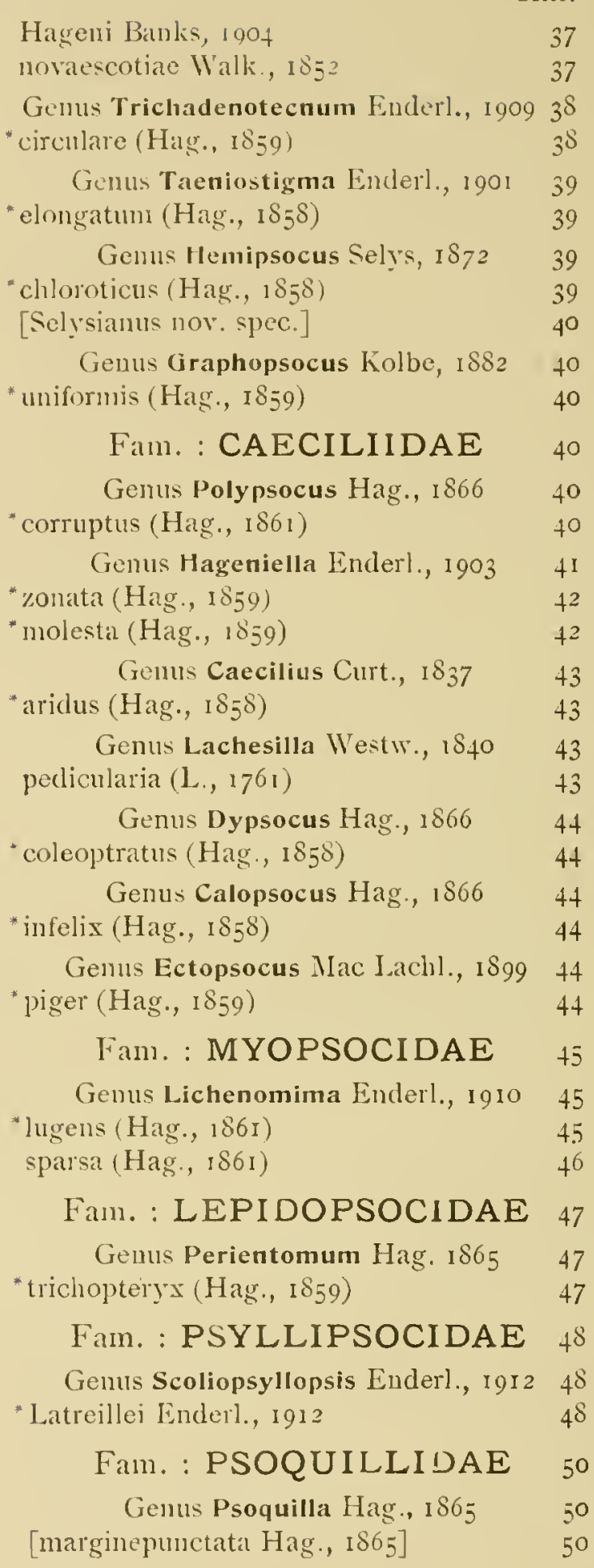




\section{ALPHABETISCHES REGISTER}

(I)ie cursiv gesetzten Nameu sind Synonyma.)

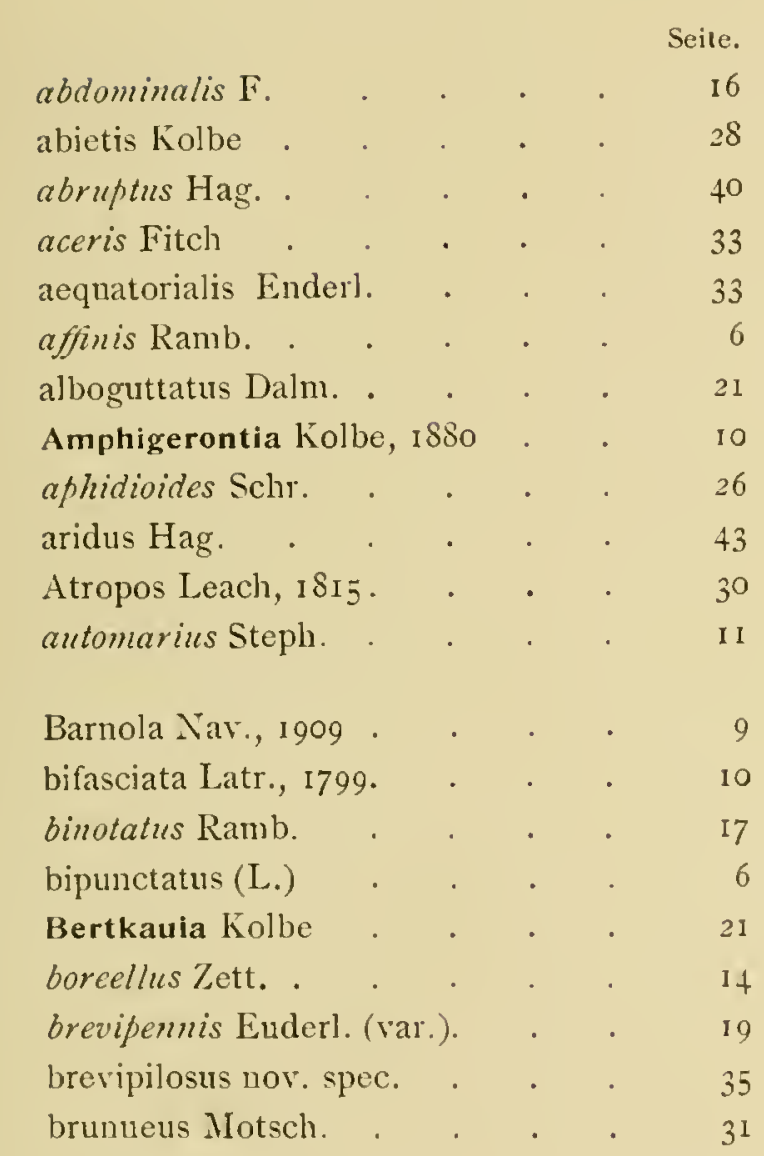

Caecilius Curt.

Calopsocus Hag.

Cerastipsocus Kolbe, 1885 . . 33

Cerastis Kolbe, $188_{3}$. . . . 33

clarus Nlac Lachl. . . . . 39

Clematostigma Enderl., I 906 . . II

chloroticus Hag. . . . . 39

coleoptratus Hag. . . . . 44

conspurcatus Ramb. . . . . 7

contaminatus Hag. . . . . 37 contaminatus Steph. . . . 10

corruptus Hag. . . . 40

costalis Steph. . . . . 13

crataegi Fitch . . . . . 37

cruciatus $(\mathrm{L}, 1768)$. . . . 13

cubanus nov. spec. . . . . 34

cubitalis nor spec. . . . $\quad 3^{6}$

Dalii (Nac Lach]., 1867) . . . 15

destructor Enclerl. . . . . 30

Dictyopsocus Enderl., ig01 · · 33

dispar Tet. . . . . $\quad 27$

divinatorius $\mathrm{L}$. . . . $\quad 3^{1}$

domesticus Burm. . . . . $\quad 17$

dubius Steph. . . . . . 17

Dypsocus Hag. . . . . 44

Ectopsocus Mac Lach , I $\$ 99$. . 44

Elipsocus Hag., 1866 . . . 27

elongatum Hag. . . . 39

exiguus Reut. . . . . . ${ }_{1} 8$

fasciata $(\mathrm{F} ., 1787)$. . . 10

fatidicum l. . . . . 16

fatidica (I. ) . . . . . I9

fatidicus Bum. . . . . $3^{1}$

fenestratus Curt. . . . . ${ }_{15}$

Ravescens Steph. . . . . I I2

favicans I. . . . . . 16

flavicans Steph. . . . . It

flavicans Zett. . . . . . 12

flaviceps Steph . . . . 28

flavidus (Steph.) . . . . I4 
formicaria Hang . . . 31

fusca Reut. . . . . . 27

fusciceps Reut. . . . . . 25

fuscipennis Burm. . . . . 34

fuscipennis Dalm. . . . . . 6

fuscofasiatus Enderl. . . . 42

fuscopterus Curt . . . 20

fuscopterus (Littr., 1799) . . . I5

geologue Walsh . . . . $\quad$ IS

gibbosus (Sul\%, I776) . . . . 5

Graphocaecilius Enderl, 1900 . . 16, 43

Graphopsocus K., ISSO . . 13, to

gregarius Harr. . . . 33

guestfalicus Kolbe . . . 30

Hageni Banks . . . . 37

Hageniella Enderl., 1903 . . . $t^{1}$

helvimacula Enderl. . . . 34

Hemineura Tetens, 1891 . . . 27

Hemipsocus Selys, is $; 2$. . 39

heteromorphus Bertk. . . . 9

hilaris Nav. . . . . 7

hirticurnis Reut. . . . . 7

hualinns Enderl. . . . . 39

Hyperetes Kolbe, 1880 . . . 30

immaculatus Steph. . . . 12

immunis Steph. . . . . 26

infelix Hag. . . . . . tt

infuscata Harr. . . . . 46

infuscalus Ramb. . . . . . 6

inquinatus Dalm. . . . . 7

irroratus Curt. . . . . 28

Lachesilla Westw., 1840 . . 15, 43

Lachlani Kolbe. . . . . 12

Lapithes Bertk., 1882 . . . 21

laricis Bremi . . . . 20

Lasiopsocus linderl., I907 • . 35

lasiopterus Burm. . . . . 28

Latreillei Enderl. . . . . 48

lepidinus Nav.. . . . . 9

Leptella Reut., 1893 . . . . $\quad 25$

Leptodella Reut., 1904 . . . 25
Leptopsocus Reut. . 16,43

Lichenomima Enderl , 1910 . 45

linealus Latr. . . . . . 5

Liposcelis Mlotsch., 1852 . . . $\quad 3^{1}$

lungicornis $\mathrm{I}$. . . . . . . 5

longicomis Steph. . . . . 26

lucifuga (Raml).) . . . . 21

lucifugus Ramb. . . . . 21

lugens Hag. . . . . . 45

luridus Enderl. (var.) . . 40

maculiponis Steph. . . . 7

magnus Walk. . . . . . 33

major Kolbe . . . . . 11

majus (Kolbe) . . . . . 11

marginepunctata Hag. . . . 50

megastigmus Steph . . . . 10

Mesopsocus Kolbe, 1880 . . . 26

microphthalmus Ramb. . . . 33

molesta Hag. . . . . 42

morio (L., I794) . . . . 11

museorum Mlotsch. . . . . 31

naso Ramb. . . . . . 26

nebulosus Steph. . . . . 5

Neopsocus Kolbe, i $\$ 82$. $\quad$. $\quad$. 9

nigricans Steph. . . . . 17

nigricomis Steph. . . . 20

nigrofasciatus Hag. . $\quad 34$

novaescotiae Walk. . . . . 37

nubilus Harr. . . . . . 45

Nymphopsocus Enderl., I 903 . . 30

obliteratus Zett. . . . . 26

obsoletus Steph. . . . . . 14

obscurus Ramb. . . . . 20

obtusus Hag. . . . . . . $3^{6}$

ochropterus Steph. . . . . 14

oculatus Sund. . . . . 26

pedicularia L . . . . . $16,+3$

penniconnis Burm. . . . . 33

Peripsocus Hag., I 866 . . 20

Perientomum Hag. 1859 . . 47

Philotarsus Kolbe, 1880 . . . 28 
Seite.

piceus Kolbe, ISS2 . . . . . I5

picicornis Steph. . . . . I0

piger Hag. . . . . . . 4

pilicornis latr. . . . . 1t

phacopterus (Mïll., 1S26) . . 20

Polypsocus Hag., I866 . . . . 40

prisca Kolbe . . . . . 21

Psocus Latr., I77t . . . . . 5, . . . . . .

Psoquilla Hag., $\mathrm{T}_{5} 6_{5}$. . . . 50

Psyllipsocus Selýs, i 872 . . . 29

Pterodella Kolbe, 1880 . . . 15

Plilopsocus Ender]., 1900. . . to

fulicarius Bertli . . . . 21

pulsatorim L. . . . . 30

pusilus Harr. . . . . . 18,73

pusillus Mïll . . . . . 16

pusillus Dalm. . . . . . 28

quadrinaculatus Latr., 1794 . . 7

quadrinaculatus Stepl. . . . 21

quadrimaculatus Westw. I $8_{4}$. . 10

quadripunctatus F. . . . 13

quercus Kolbe, $\mathrm{I} S 82$. $\quad$. $\quad$ I 9

quinquepunctatus Mac Lach]. . . $\quad 38$

Ramburi Selys . . . . . $\quad 29$

ramosus Walk. . . . . . 33

Reuterella Enderl., I 903 . . . 23

rhenanus Kolbe, $188_{2} \quad$. . . 9

rufescens Steph. . . . . . 12

salicis Hag. . . . . . 18

saltatrix L. n. 11. . . . . 5

Scoliopsyllopsis Enderl., 1912. . $\quad 18$

selysianus nov. spec. . . . $t 0$

sexpunctatum $(\mathrm{I}$, ) . . . II

silvarum kiolbe. . . . . 31

sparsa"Hag. . . . . . . 46

similis Steph. . . . . . . 6

speciosus Aaron . . . 34

speciosus burm. . . . $\quad 3^{2}$
Spinolne Ramb. . . . 33

Stenopsocus Hag., 1866 . . 12

stigmaticus (lmh. et 1.ahr., is 66 . I 13

strialulus Steph. . . . . . 28

striatulus li. . . . . . I3

striatus Dalm. . . . . . It

strigosus Curt. . . . . . . I7

strigosus Bum. . . . . . 12

sludiosa L. . . . . . 30

subfasciatus Ramb. . . . . 20

subfasciatus \%ett. . . . . . IO

subfumipennis \%ett . . . . . . 12

subuebulosus Steph. . . . . . 7

subocellatus Steph. . . . . . 13

subpunctatus Steph. . . . . If

subpupillatus Mac Lach. . . . 20

Taeniostigma Enderl., I901 . . 39

titprobanes Hag. . . . . 36

Thyrsophorus Burm., $18_{5} 8$. . 32

Thyrsopsocus Enderl., r901 . . 33

tolteca Banks . . . . . 34

Trichodenotecnum Ender?., 1909 . II, 38

Trichopsocus Kolbe, I 888 . . I5

trichopteryx Hag. . . . . . 47

trifasciatus Prov. . . . . 34

Troctes Burm. . . . . . $3 I$

Trogium 111., 1798 . . . . 30

unjformis Hag. . . . . 40

unipunctatıs Müll. . . . . . 26

variegata (Iatr., I799) . . . II

wariegatus Curt. . . . . . 6

venosus Burm. . . . . 33

venosus Steph. . . . . . I2

vitripennis Curt. . . . . $\quad 26$

villalus Dalm. . . . . . 15

Westwoodi Mac Lachl. . . . $\quad 27$

zonatil Hag. . . . . . $t^{2}$ 


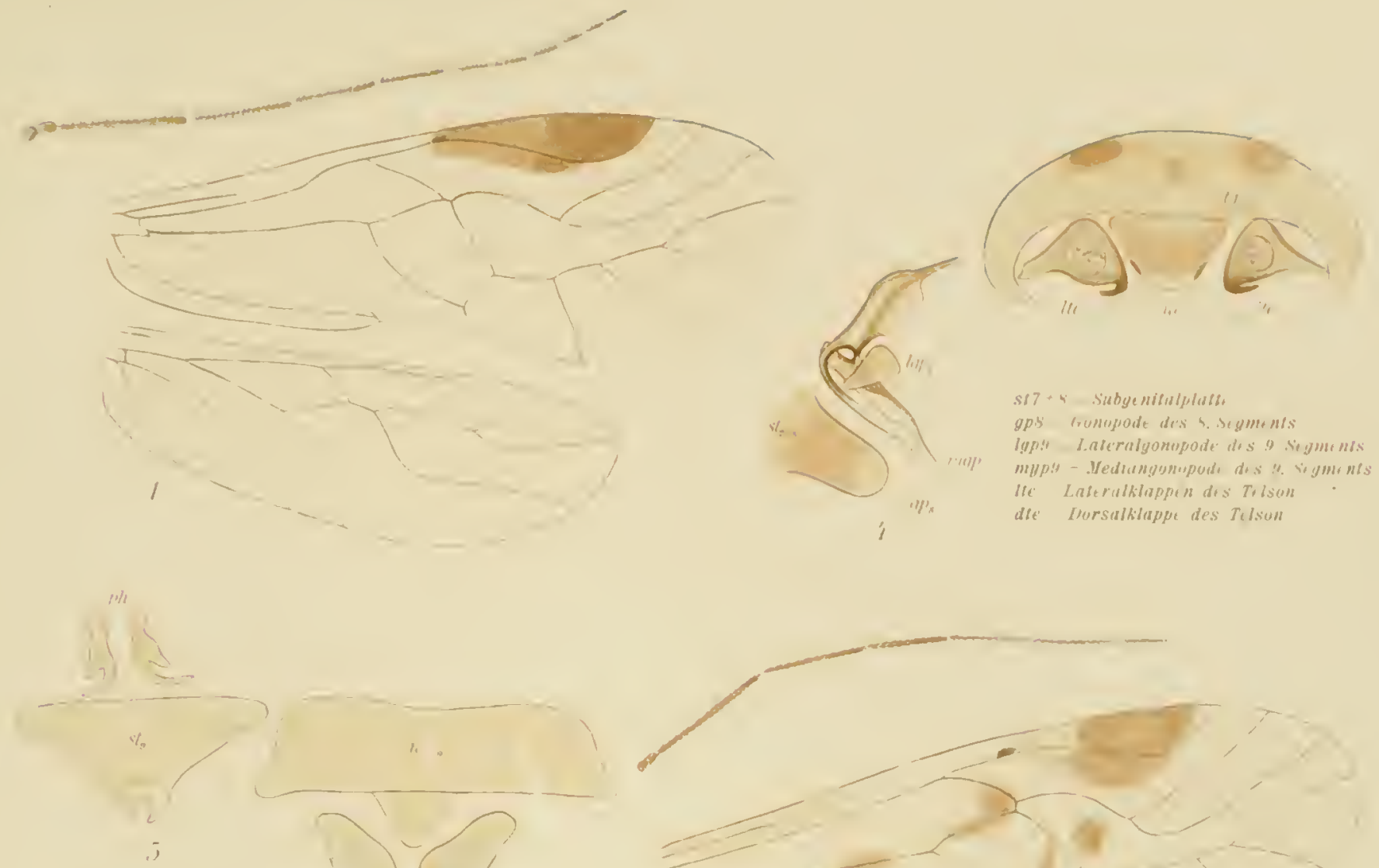

ph - Penishaten

stg-Subgenitalplatte

lte $=$ Lalerulklappen des Telsan
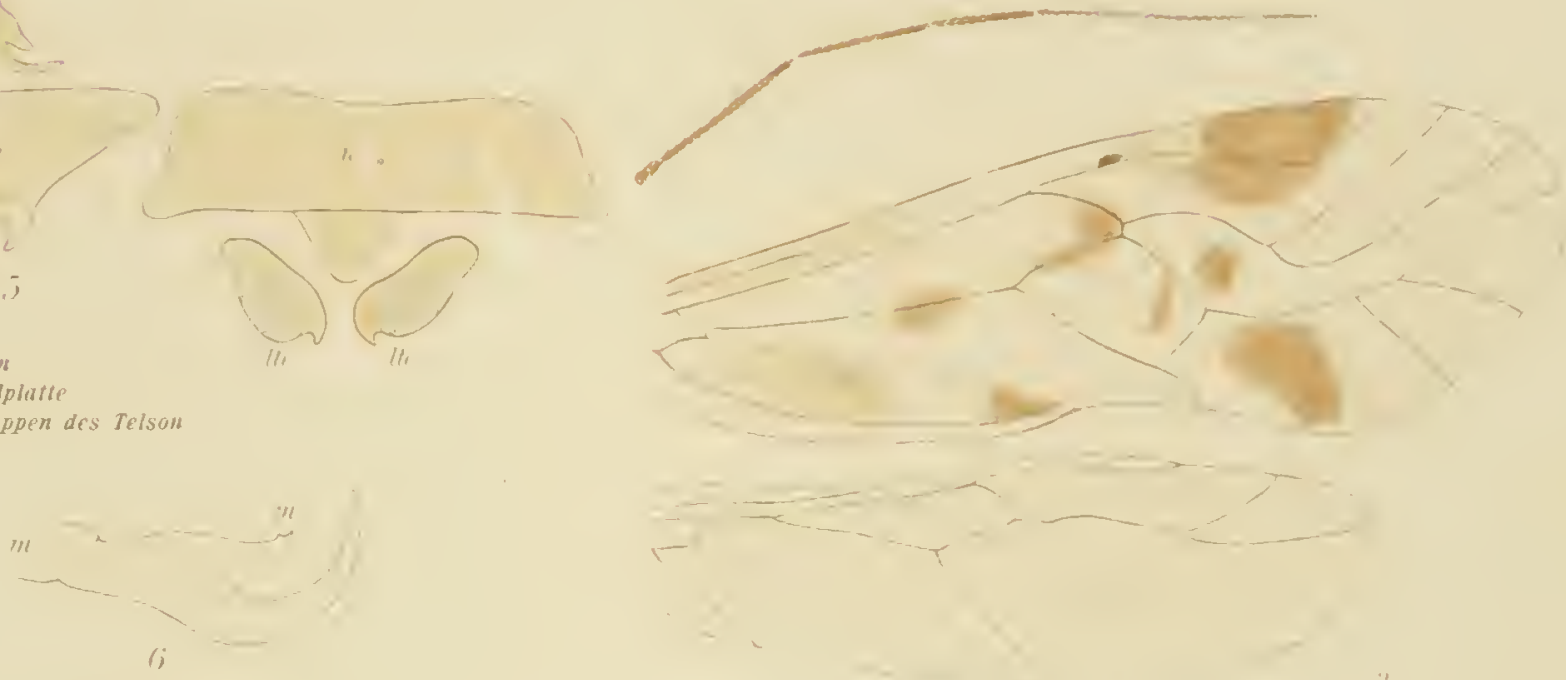

III Muskelansilize
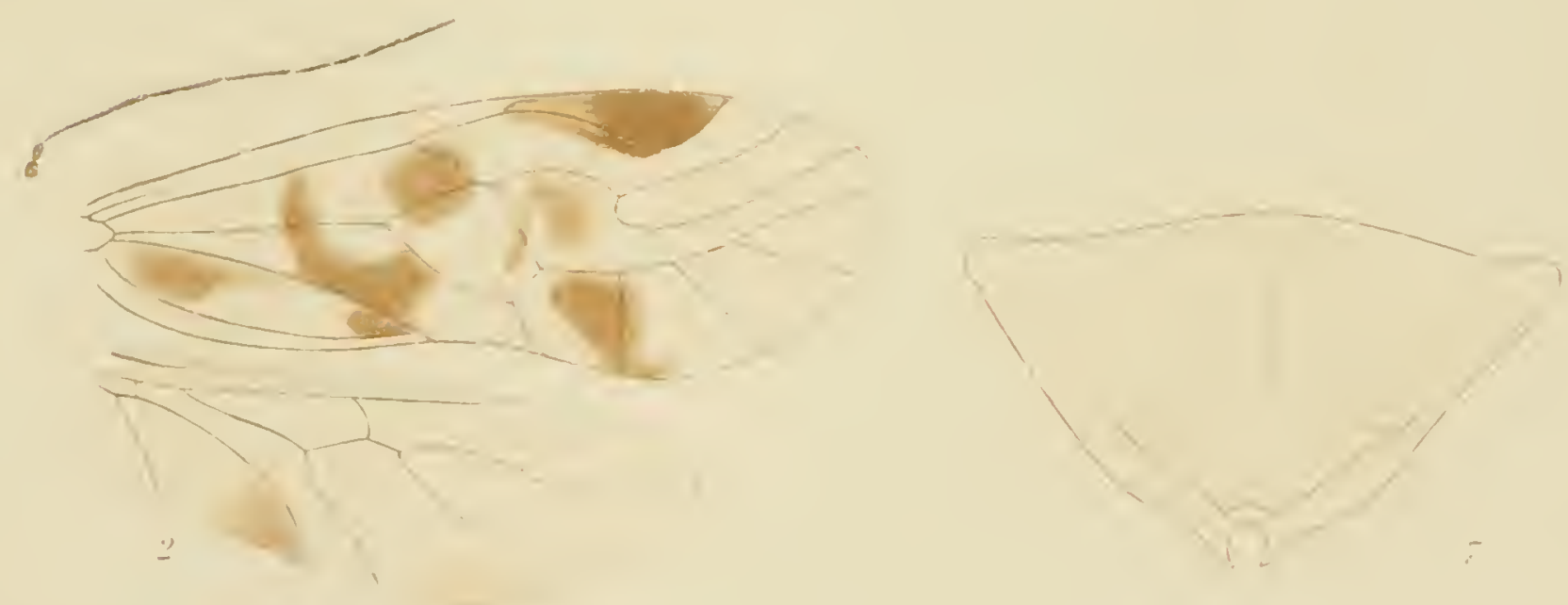

Psocus quadrimaculatus Latr.

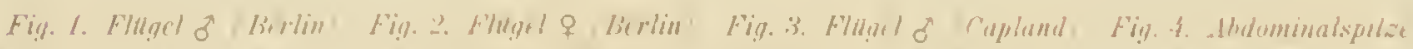

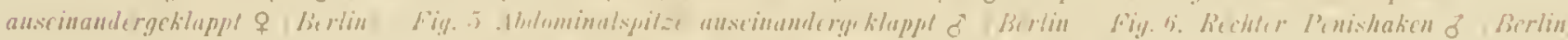
Fivg. i. sulngenilulplalle und Penishutien of Copland. 


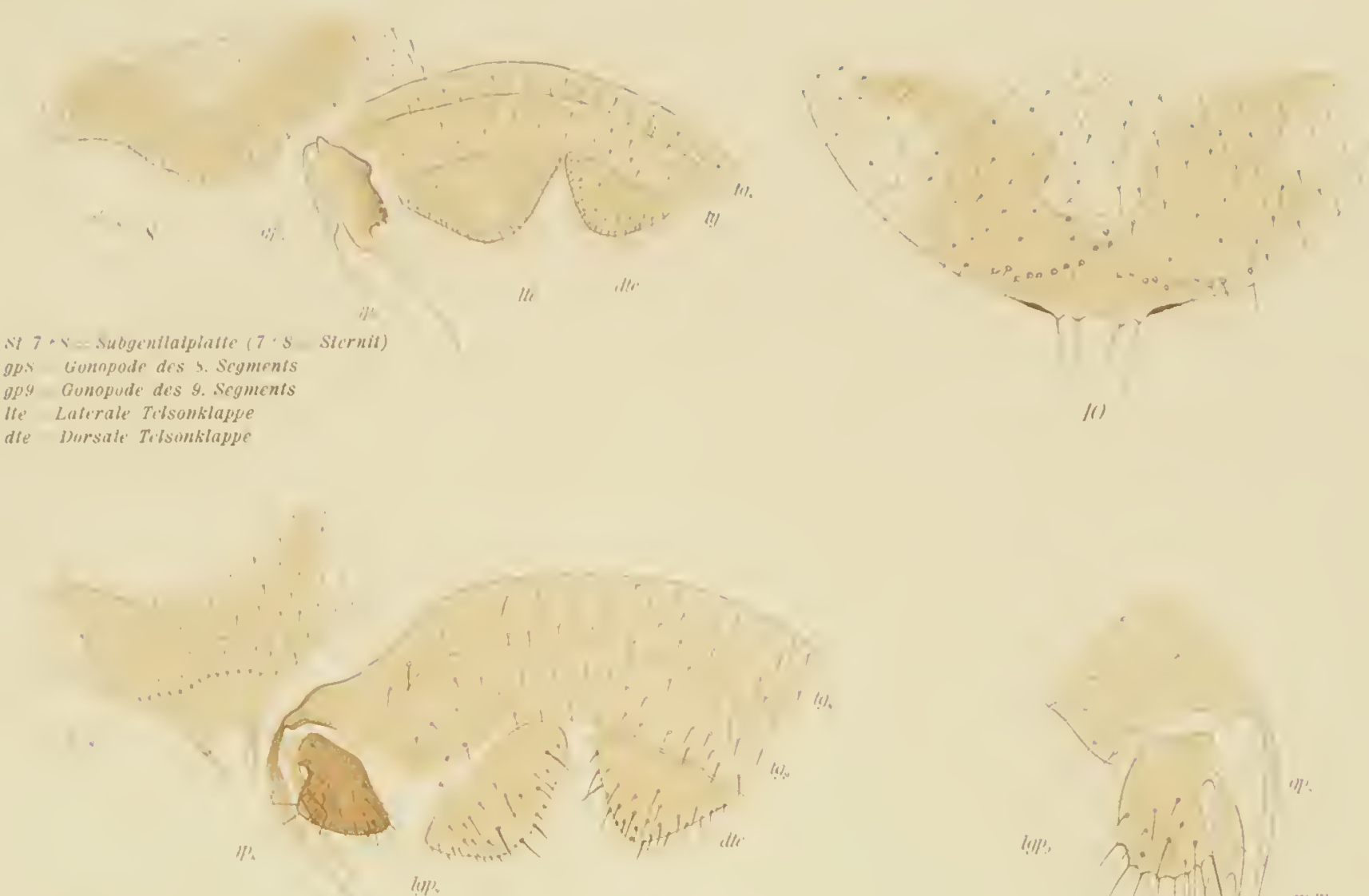

st7 - s subgenilalplatle $(7 .+8$. Sternit) gp. - Gunoptude des 8. Segments

mup.9 - Vediangonopode des 9. Segments

lgp, $=$ Lattralgonopode des 9. Segments

lle = Lateralklappe des Telsan

die = uorsalslappe des Telsan
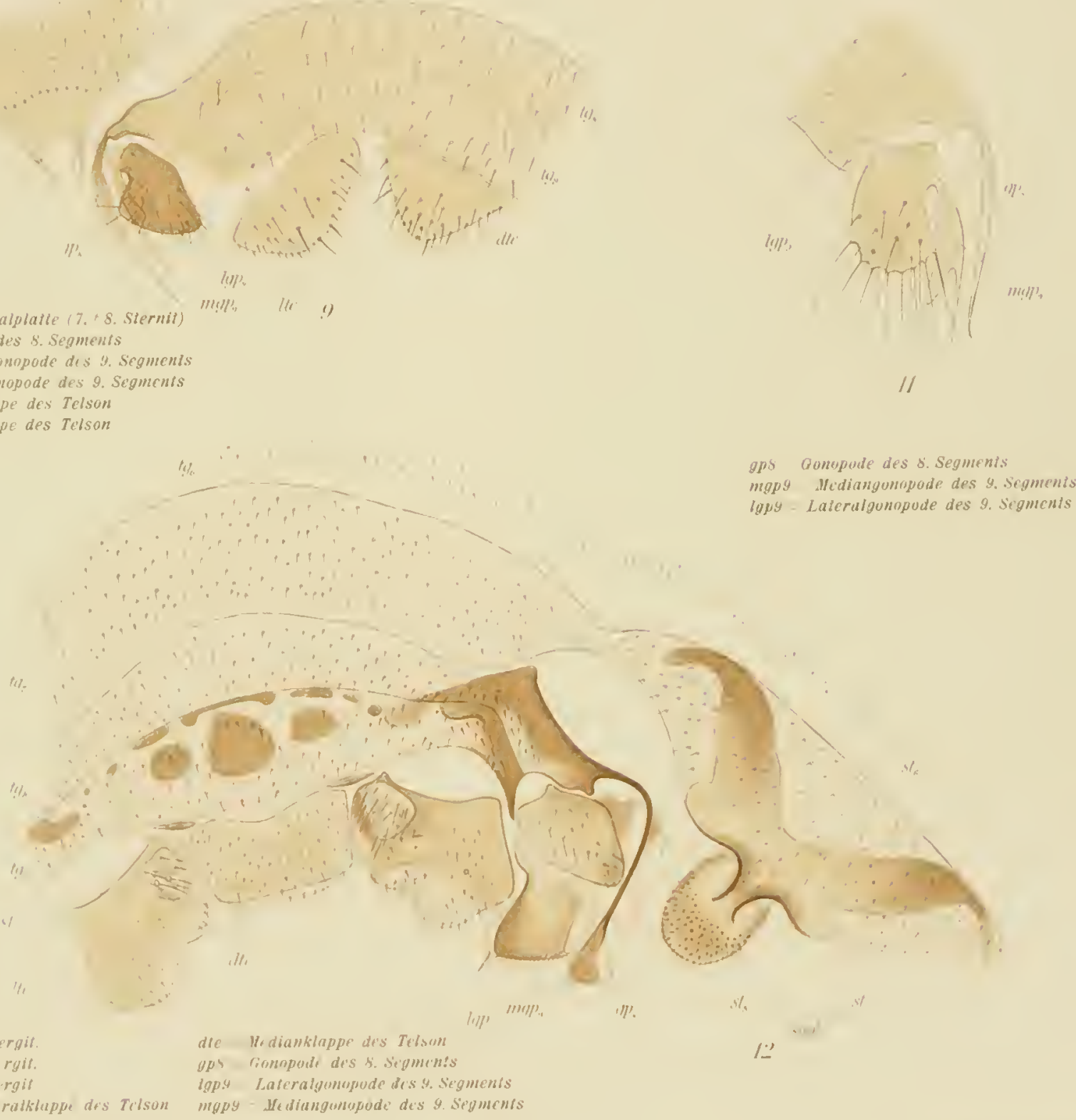

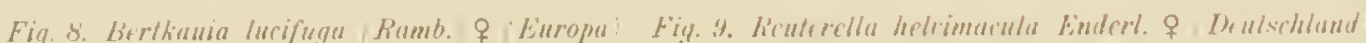
Fig. 10. Leplodella fusctepss Reul.) o Finnland Fiy. 11. Lepdodella fuscicups, heut. of Finnland

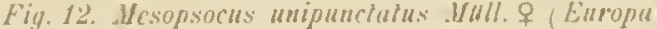


. 

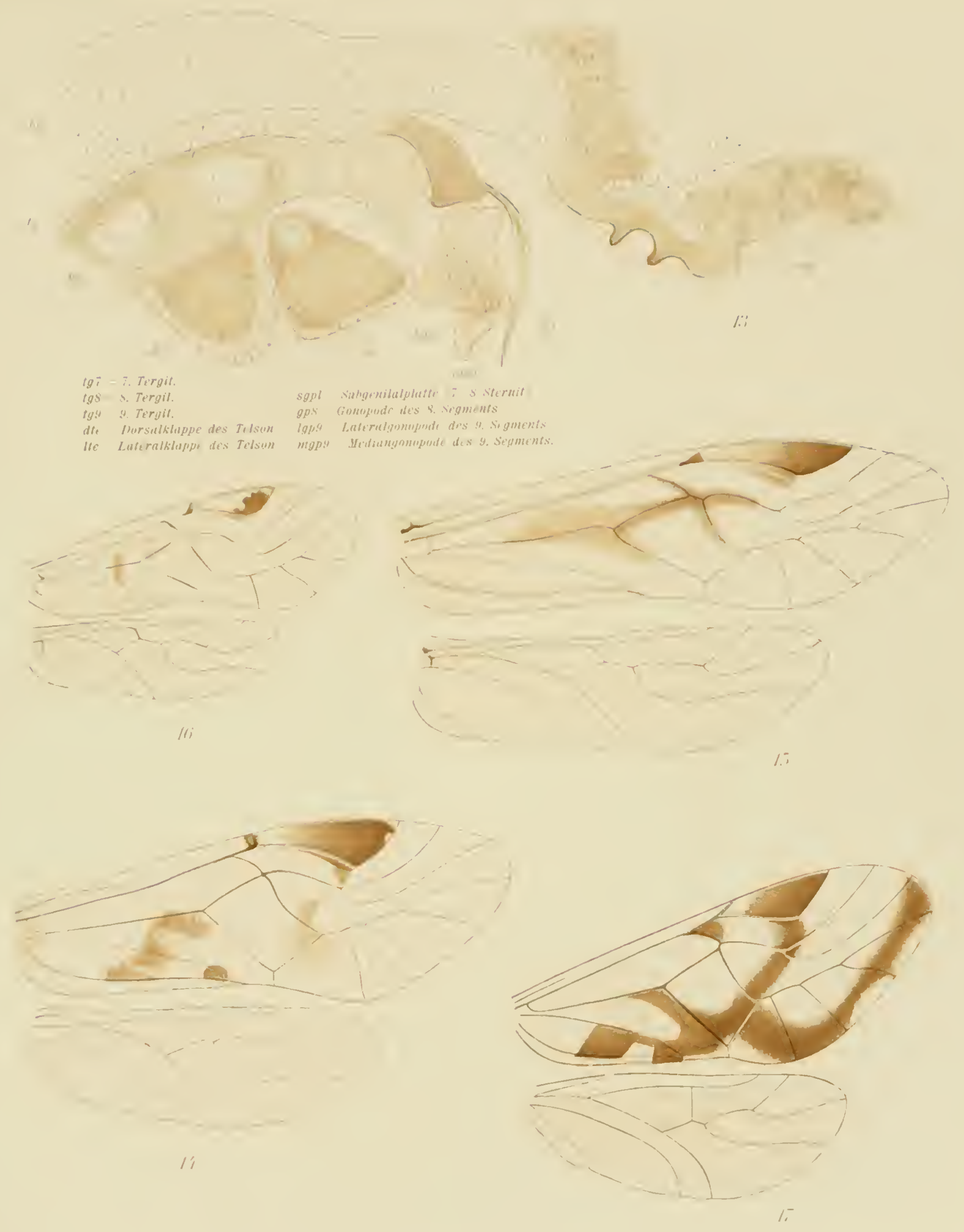

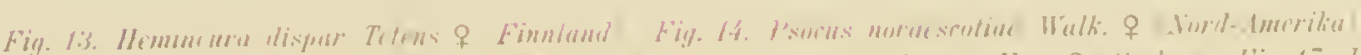

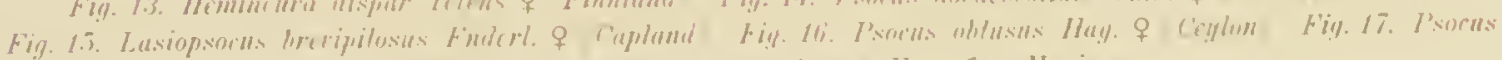

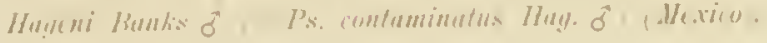





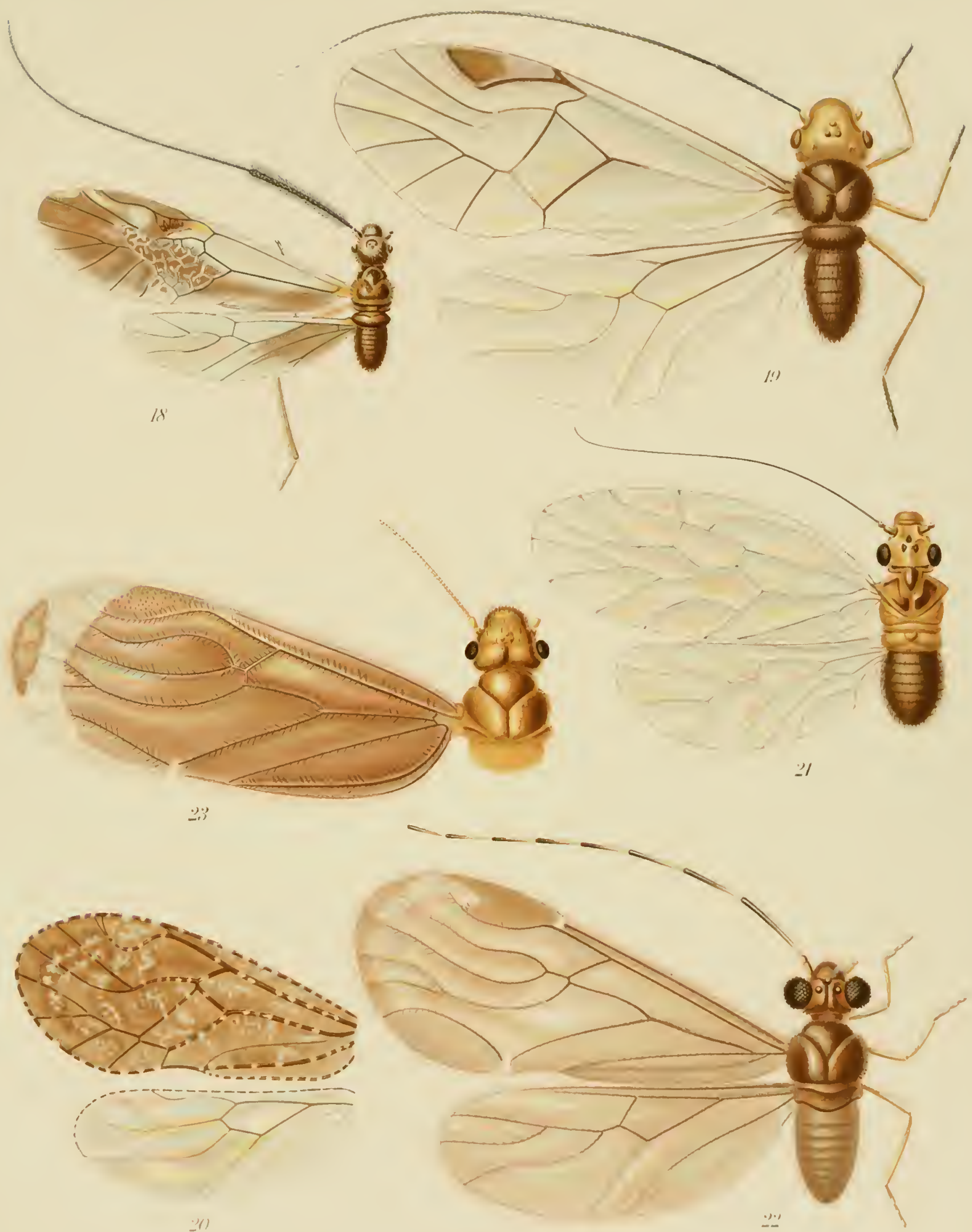





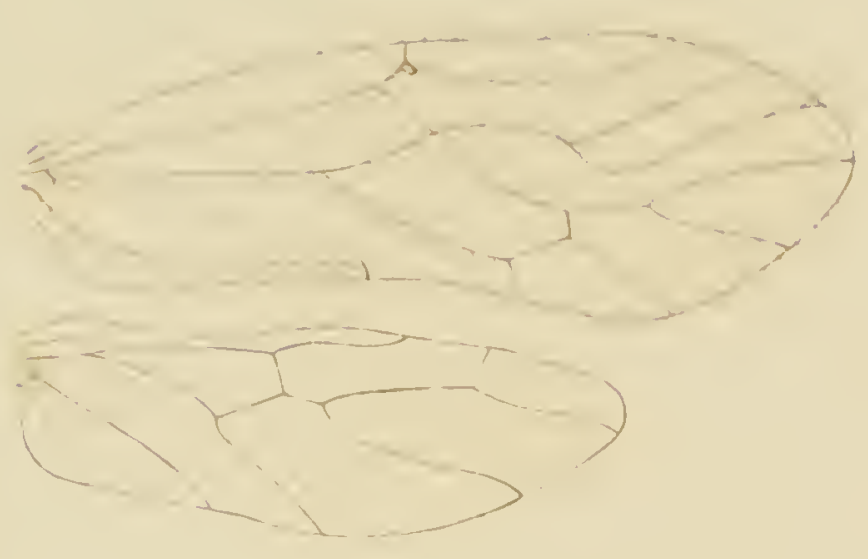

$\stackrel{m !}{\prime \prime}$
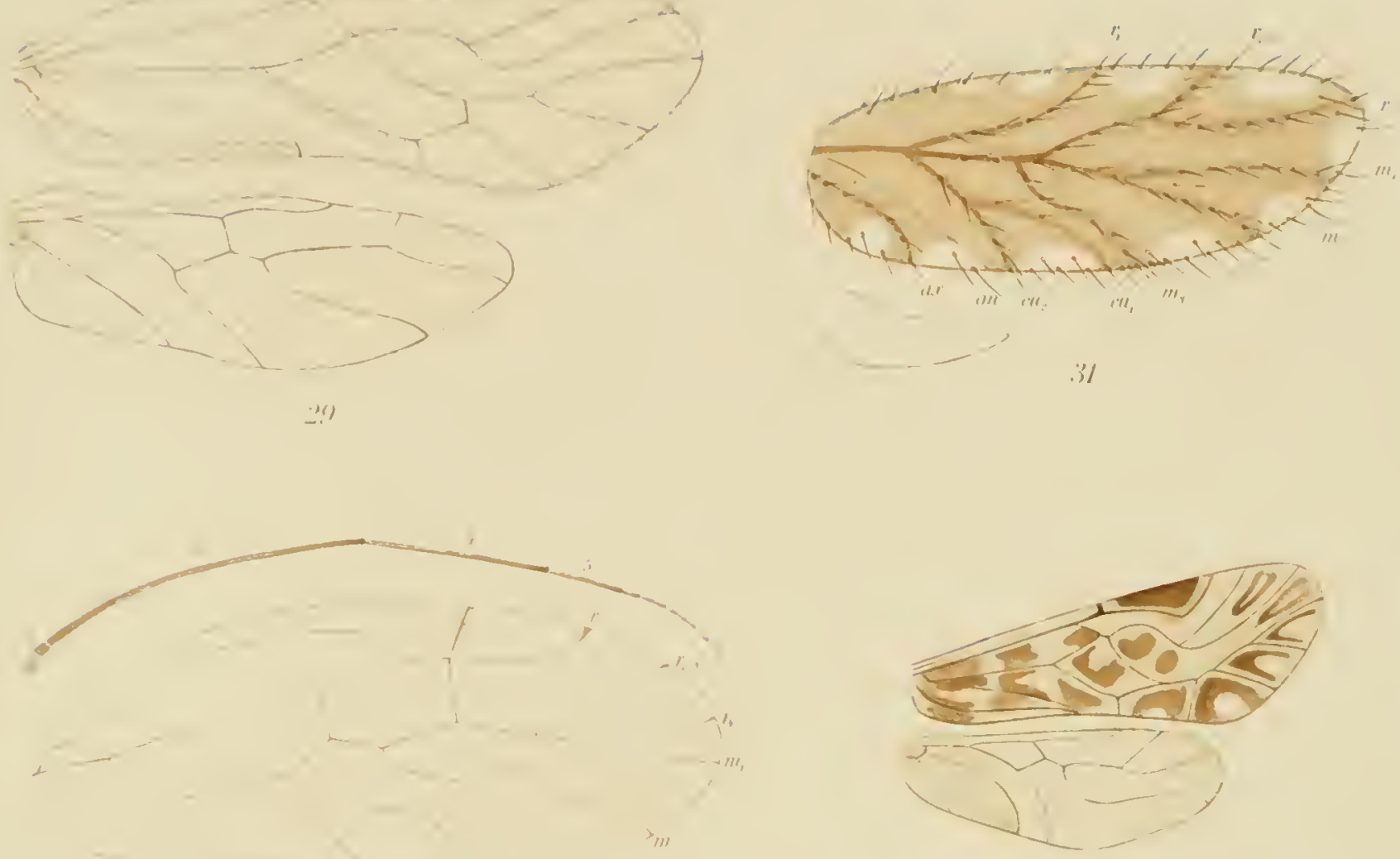

$2\}$
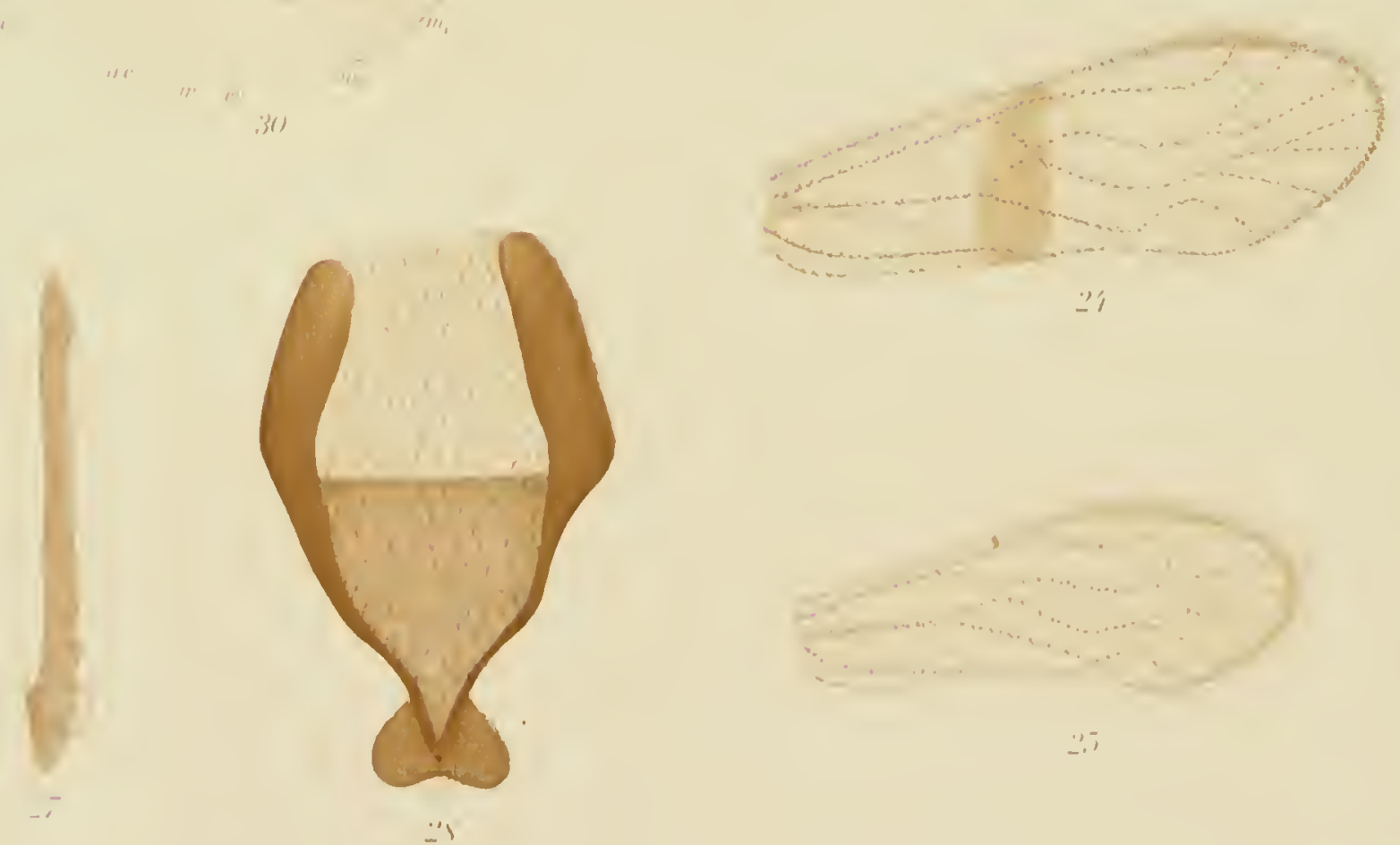

$\stackrel{9}{2}$

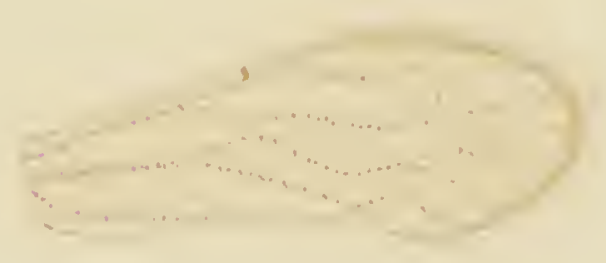

$\therefore ;$ 


